\title{
LE ELEZIONI COMUNALI IN TOSCANA DAL 1993 AL 2004. GLI EFFETTI DELLE NUOVE REGOLE, I PARTITI, LE COALIZIONI, I "NUOVI" SINDACI
}

\author{
di ANTONIO FLORIDIA
}

Una prima fase di questa ricerca, relativa alle elezioni comunali fino al 2003, è stata svolta dall'autore presso l'IRPET, su incarico del Consiglio della Autonomie locali della Toscana ed è stata pubblicata, a cura dello stesso Consiglio delle Autonomie locali, nel maggio 2004. La fase successiva della ricerca qui pubblicata e comprendente anche le elezioni del 2004, è stata svolta nell'ambito delle attività dell'Ufficio e Osservatorio elettorale della Regione Toscana. La creazione del data base e le elaborazioni statistiche sono state curate da Lorenzo De Sio. I dati elettorali utilizzati sono, in genere, tratti da fonti del ministero dell'Interno. In alcuni casi, tuttavia, essi sono stati integrati e verificati con dati provenienti da altre fonti: in primo luogo, la stessa banca dati dell'Osservatorio elettorale, a sua volta integrata e aggiornata in collaborazione con gli uffici elettorali dei Comuni. 


\section{Introduzione}

L'obiettivo di questo lavoro quello di fornire un bilancio critico e una valutazione degli effetti che l'applicazione della legge 81 del 25 marzo 1993 ha prodotto sui sistemi politici locali della Toscana: una legge che è stata più volte, e da più parti, definita come il frutto migliore della convulsa fase di crisi e di transizione che hanno vissuto le istituzioni politiche del nostro paese nel corso degli anni Novanta, - «un esempio di ingegneria istituzionale sostanzialmente andato a buon fine», come è stato detto ${ }^{1}$-, ma di cui oggi, a distanza di anni, e dopo tre cicli elettorali, si possono cominciare a cogliere meglio le complesse implicazioni, individuando i processi di mutamento che le nuove regole hanno indotto, o con i quali esse si sono intrecciate, nel comportamento degli elettori e nelle strategie degli attori politici.

Ad oltre dodici anni dall'approvazione di questa legge, tutti i Comuni hanno già sperimentato per almeno tre volte il nuovo sistema elettorale. È possibile quindi disporre oggi di una mole rilevante di dati che permettono di valutare le modalità di funzionamento del nuovo sistema elettorale. Concentrare poi l'attenzione su una sola regione, la Toscana nel nostro caso, corrisponde ad un esigenza conoscitiva di particolare rilievo, proprio perché, come rilevava Giacomo Sani nella Prefazione ad uno degli studi più completi sull'argomento, apparso dopo la tornata elettorale del 1999, ciò che emerge sono appunto «le forti differenze a base regionale» che hanno caratterizzato l'impatto della nuova legge elettorale ${ }^{2}$.

1 S. VASSALlo, Sistema elettorale, in Organi e sistema elettorale. Commenti al T.U. sull'ordinamento degli enti locali, a cura di L. Vandelli, T. Tessaro, S. Vassallo, Rimini, Maggioli editore, 2001, pp. 726.

2 G. SANI, Prefazione a G. Baldini e G. Legnante, Città al voto. I sindaci e le elezioni comunali, Bologna, Il Mulino 2000. Per una breve rassegna degli studi e delle ricerche sulla nuova legge elettorale comunale, si rimanda alla Nota bibliografica, proposta a conclusione di questo nostro lavoro. 
In questa ricerca, dunque, siamo partiti dalle specificità del sistema politico toscano, cominciando ad analizzare alcuni aspetti del voto amministrativo nel corso degli anni Ottanta e cercando di misurare, per quanto possibile, i mutamenti che il nuovo sistema elettorale ha prodotto. I comuni considerati dalla ricerca sono quelli che, sulla base dei dati del Censimento 1991, e poi di quello del 2001, hanno superato i 15 mila abitanti e per i quali, dunque, la nuova legge prevede un sistema elettorale a doppio turno: per la Toscana, si tratta di 51 comuni ${ }^{3}$. Per questi comuni è stato predisposto un data base completo, oggi anche consultabile via internet ${ }^{4}$, comprendente tutti i dati relativi alla partecipazione elettorale, i voti riportati dai candidati e dalle liste collegate, i seggi attribuiti a ciascuna lista. Generalmente, i dati sono stati analizzati anche sulla base delle diverse classi demografiche dei comuni e della diversa ampiezza dei consigli comunali ${ }^{5}$, e sulla base dei diversi cicli elettorali, in modo da consentire confronti omogenei ${ }^{6}$.

Il primo tema affrontato è quello della partecipazione elettorale in Toscana, dapprima in una prospettiva temporale di lungo periodo, mettendo in luce i rilevanti mutamenti intervenuti nel corso degli anni Novanta, soprattutto per quanto riguarda le differenziazioni nei livelli di partecipazione al voto, tanto tra i diversi tipi di elezione (politiche, regionali e amministrative) quanto tra le diverse, possibili modalità di espressione del voto anche in occasione della stessa consultazione elettorale. Una particolare attenzione sarà poi dedicata al feno-

3 Riportiamo qui l'elenco completo dei comuni che sono stati oggetto della nostra analisi, distinti per provincia: Arezzo: Arezzo, Cortona, Montevarchi, San Giovanni Valdarno, Sansepolcro. Firenze: Firenze, Bagno a Ripoli, Borgo San Lorenzo, Campi Bisenzio, Castelfiorentino, Certaldo, Empoli, Fiesole, Figline Valdarno, Fucecchio, Impruneta, Lastra a Signa, Pontassieve, San Casciano Val di Pesa, Scandicci, Sesto Fiorentino. Grosseto: Grosseto, Follonica. Livorno: Livorno, Cecina, Collesalvetti, Piombino, Rosignano Marittimo. Lucca: Lucca, Camaiore, Capannori, Massarosa, Pietrasanta, Viareggio. Massa Carrara: Massa, Carrara. Pisa: Pisa, Cascina, Pontedera, San Giuliano Terme, San Miniato. Pistoia: Pistoia, Monsummano Terme, Montecatini Terme, Pescia, Quarrata. Prato: Prato, Montemurlo. Siena: Siena, Colle Valdelsa, Poggibonsi. La maggior parte di questi comuni ha votato in occasione dei turni principali di elezioni amministrative svoltesi in questo decennio (1995-1999-2004); ma quindici di essi hanno cominciato a votare con il nuovo sistema elettorale già nel 1993 e nel 1994: si tratta, peraltro, di ben sei capoluoghi di provincia (Massa, Pisa, Lucca, Grosseto, Siena e Pistoia) e poi di Montevarchi, Figline Valdarno, Camaiore, Pietrasanta, Viareggio, Carrara, Pescia, Quarrata e Cascina. Due comuni (Calenzano e Signa), a seguito del censimento del 2001, hanno superato la soglia dei 15 mila abitanti e quindi hanno votato per la prima volta nel 2004 con il nuovo sistema elettorale. Al contrario, i comuni di Impruneta e Fiesole sono scesi sotto questa soglia, e quindi saranno considerati nella nostra analisi solo per le due precedenti elezioni. Per il terzo ciclo elettorale, i casi considerati sono 52, in quanto il comune di Pescia, al voto nel 2001, è stato richiamato anticipatamente alle urne nel 2004, per le dimissioni del sindaco e lo scioglimento anticipato del consiglio.

4 Il data base è consultabile presso il sito dell'Osservatorio elettorale della Regione Toscana (www.regione.toscana.it, all'interno della sezione "La comunicazione istituzionale").

5 Com'è noto, la legge 81 ha modificato l'ampiezza dei Consigli comunali: si veda, qui di seguito, il paragrafo 3 .

6 I cicli elettorali sono quelli del 1983-1985, del 1988-1990, del 1993-1995, del 1997-1999 e, infine, del 2000-2004. Com'è noto, a seguito delle modifiche introdotte dalla legge n. 120 del 1999, la durata del mandato del sindaco e del mandato consiliare è stata portata da quattro a cinque anni. 
meno del "voto personalizzato", ossia al voto che viene espresso solo ai candidati sindaci, e non anche alle liste di partito. Verranno inoltre analizzati i dati sull" astensionismo aggiuntivo" in occasione dei ballottaggi e, infine, verranno proposti alcuni primi dati sull'uso del voto di preferenza per la scelta dei candidati consiglieri, introducendo dunque, tra le diverse modalità di espressione del voto, anche la distinzione tra voto esclusivo alle liste di partito e voto alle liste con preferenza.

Successivamente saranno toccati tutti quegli aspetti che vengono solitamente accomunati sotto la definizione di offerta politica ed elettorale: in particolare, verranno analizzati $i$ dati relativi al numero e alla media delle liste concorrenti e al numero e alla media delle liste che riescono ad essere rappresentate nei consigli comunali, con un confronto tra le elezioni amministrative degli anni Ottanta e quelle svoltesi successivamente all'introduzione del nuovo sistema elettorale. Verranno poi analizzate anche le tipologie delle liste, ossia le caratteristiche "nazionali" e/o locali dell'offerta politica (presenza di partiti nazionali con il proprio simbolo e/o liste locali o civiche) e, soprattutto per le elezioni post-1993, si distinguerà tra le liste di coalizione tra partiti "nazionali", le liste di coalizione locale tra partiti nazionali con simbolo locale o riferimento "civico", le liste civiche e/o locali presenti nelle coalizioni maggiori e le liste locali "isolate". Potremo avere così un quadro completo della progressiva differenziazione dell'offerta elettorale, indotta dai mutamenti complessivi dello scenario politico nazionale, ma anche sollecitata dagli specifici meccanismi elettorali previsti dal nuovo ordinamento.

Legato al tema dell'offerta, vi è quello del livello effettivo di rappresentanza: gli effetti indotti dal nuovo sistema elettorale verranno misurati quindi sulla base di un calcolo della soglia di accesso ai consigli comunali, nei diversi cicli elettorali e per le diverse dimensioni dei consigli. Si tratta qui di verificare se ed in che misura il nuovo sistema elettorale abbia innalzato, o meno, la soglia percentuale minima di voti con cui, (di fatto, al di là delle soglie "legali"), una lista riesce ad ottenere almeno un seggio. Naturalmente, per le elezioni successive al 1993, verrà introdotta una distinzione tra la soglia necessaria alle liste che fruiscono del premio di maggioranza, in quanto legate al sindaco vincente, e quella invece necessaria per le liste collegate a candidati sindaci perdenti.

Per valutare, infine, i mutamenti indotti dal nuovo sistema elettorale verranno presentate e analizzate alcune misurazioni specifiche sul grado di concentrazione e/o frammentazione dei sistemi politici locali. Si tratterà cioè di comprendere se, al di là del numero di liste concorrenti e/o rappresentate, i sistemi politici locali abbiano vissuto una peculiare evoluzione nel senso di una maggiore concentrazione del numero di partiti effettivamente rilevanti, tanto per ciò che riguarda il voto degli elettori (il voto è più disperso o più concentrato, rispetto al passato?), quanto per ciò che concerne le stesse assemblee elettive (la rappresentanza è più, o meno, frammentata?). 
A questo tema è poi legata una valutazione finale sull'evoluzione in senso bipolare dei sistemi politici locali: l'analisi dell'indice di bipolarismo, ossia la percentuale dei voti riportati dai primi due candidati, e poi dalle prime due liste, ci consentirà di misurare fino a che punto si sia spinta una tale evoluzione, ma anche di porre alcuni interrogativi sulle tensioni che permangono e sulle contraddizioni di tale processo.

La ricerca si concentra poi su alcuni temi per i quali non è più possibile operare dei confronti con la situazione pre-riforma: l'offerta elettorale, evidentemente, con l'introduzione dell'elezione diretta del sindaco, si arricchisce, rispetto al passato, di una nuova variabile, ossia la figura dei candidati alla carica di "primo cittadino". Da questo punto di vista, si tratterà di misurare e analizzare il numero e la media dei candidati presenti nei diversi cicli elettorali, la media delle liste presentate a sostegno dei candidati, il rapporto tra candidati e liste. Anche in questo caso, inoltre, come in precedenza per le liste concorrenti e per le liste rappresentate, si tratterà di verificare il grado di concentrazione e/o frammentazione dell'offerta, ossia verificare quanti sono stati i candidati "effettivamente" rilevanti nel caratterizzare la struttura della competizione.

Nell'ultima parte del lavoro, viene infine affrontato uno dei temi più rilevanti e più discussi, ossia il grado di personalizzazione della competizione politica ed elettorale. Verranno presentati i dati sul livello di voto "personale" dei candidati sindaci, (indice di personalizzazione: ossia, la percentuale di voti "personali" riportati dai candidati, rispetto ai voti delle liste di sostegno) e sulle capacità di "attrazione" dei vari candidati (indice di attrazione: ossia, la percentuale di voti "personali" di ciascun candidato rispetto al totale dei voti "personali" e "divisi" espressi dagli elettori): attraverso queste misurazioni, confrontate per i diversi cicli elettorali, si potrà verificare se il fenomeno della personalizzazione sia stata legato ad una peculiare fase politica (le prime prove della nuova legge elettorale), se esso perduri nel tempo ed in che misura, se esso sia legato all'uno o all'altro schieramento politico, e a quali variabili sistemiche esso sia legato (in particolare, il numero delle liste collegate e il grado di strutturazione del sistema dei partiti). Al tema dei sindaci è legato anche l'analisi del fattore incumbency, ossia la valutazione della forza elettorale e politica dei sindaci uscenti e di quanto essa si rifletta negli esiti elettorali e negli indici di personalizzazione e attrazione dei candidati stessi. Viene infine presentato un quadro riassuntivo delle coalizioni vincenti nei diversi cicli elettorali in Toscana, dei casi di alternanza tra gli schieramenti, e della composizione delle coalizioni.

Nelle conclusioni proporremo un primo bilancio degli effetti prodotti dalla nuova normativa elettorale, con particolare riferimento all'evoluzione dei sistemi politici locali della Toscana e ai modi con cui il nuovo contesto istituzionale ha interagito con le trasformazioni del modello politico e sociale che, storicamente, ha caratterizzato questa regione. 


\section{La partecipazione elettorale}

Voto politico e voto amministrativo: $i$ divari nei livelli della partecipazione. - Nella storia politica italiana della seconda metà del Novecento, la Toscana si è sempre caratterizzata come una delle regioni con i più alti livelli di partecipazione elettorale: nelle elezioni politiche, la percentuale dei votanti in Toscana si è mantenuta costantemente più elevata di quella media nazionale e, anche negli anni in cui ha cominciato a manifestarsi in modi più vistosi il fenomeno dell'astensionismo, la Toscana (che pure è stata investita dalla stessa tendenza) ha comunque conservato intatto un divario positivo, rispetto al dato complessivo (FIG. 1). Tali altissimi livelli di partecipazione sono stati a lungo interpretati come una delle espressioni tipiche di una regione che, al pari di altre regioni del Centro e del Nord-Est del nostro paese, era caratterizzata dalla prevalenza di una delle due grandi "subculture politiche territoriali" che hanno dominato la scena politica del dopoguerra italiano. Secondo questa interpretazione, l'esistenza di una forte identità collettiva e di una cultura politica, socialmente maggioritaria, orientata al sostegno di una determinata forza politica, e articolata attraverso una fitta rete organizzativa e istituzionale, si traduceva in un alto, e costante, livello di mobilitazione politica e, quindi anche in una forte motivazione alla partecipazione elettorale. Il momento del voto si configurava, in questo schema, come uno dei canali privilegiati di espressione di un'appartenenza politicoideologica radicata nella storia e nella comunità locale. Si tratta di una chiave interpretativa che ha retto a lungo e che ha trovato numerosi e convincenti momenti di verifica nella storia politica ed elettorale dell'Italia repubblicana.

FIG. 1. - Percentuale dei votanti nelle elezioni per la Costituente e per la Camera dei deputati in Italia e in Toscana.

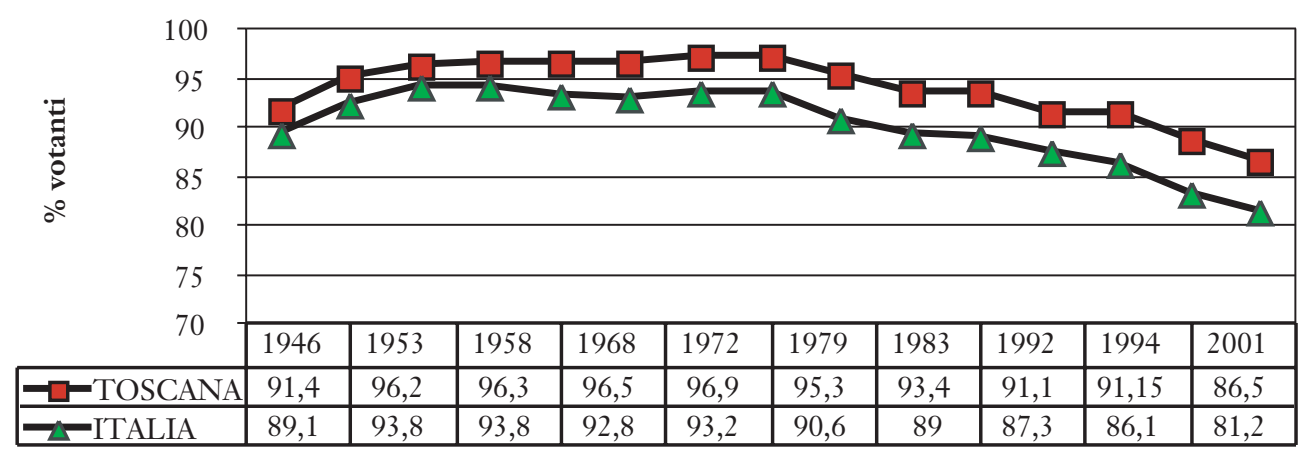

Tuttavia, a partire dagli anni Ottanta e, in modo sempre più evidente poi nel corso degli anni Novanta, i mutamenti nella cultura politica del nostro paese, la crisi del vecchio sistema politico e gli stessi processi di erosione e di trasforma- 
zione che investivano le grandi tradizioni politico-culturali del nostro paese, cominciano a riflettersi apertamente nelle forme e nei livelli della partecipazione elettorale: da un lato, attraverso una caduta generale della partecipazione; dall'altro, e soprattutto, per quanto qui ci interessa più direttamente, attraverso una crescente differenziazione nelle percentuali dei votanti ai diversi tipi di elezione (europee, politiche, regionali e amministrative: si vedano la FIG. 2 e la TAB. 1) ${ }^{7}$.

FIG. 2. - Toscana. Percentuale dei votanti alle elezioni politiche, regionali ed europee.

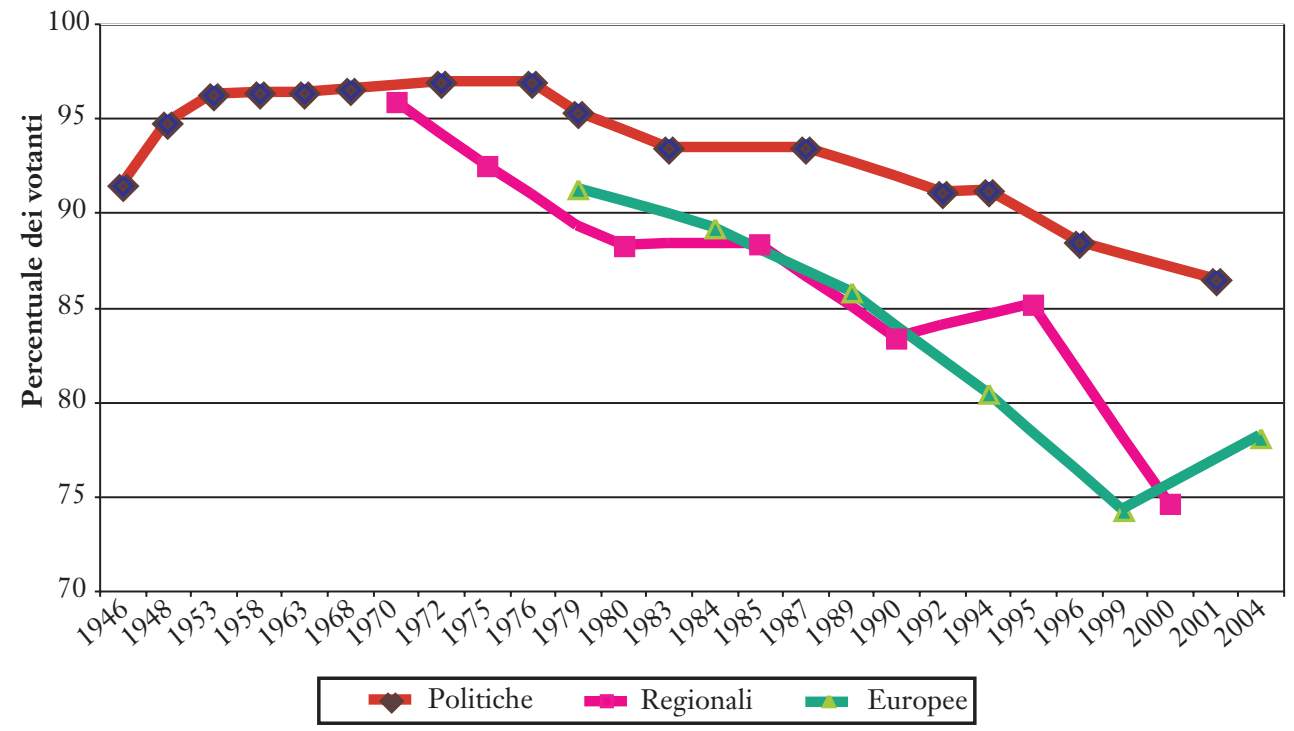

TAB. 1. - Toscana. Percentuale dei votanti alle elezioni politiche, regionali ed europee.

\begin{tabular}{lccccccccc}
\hline & 1946 & 1948 & 1953 & 1958 & 1963 & 1968 & 1970 & 1972 & 1975 \\
\hline $\begin{array}{l}\text { Politiche } \\
\text { Regionali } \\
\text { Europee }\end{array}$ & 91,4 & 94,7 & 96,2 & 96,3 & 96,3 & 96,5 & & 96,9 & \\
\hline & & & & & & & 95,9 & & 92,5 \\
\hline $\begin{array}{l}\text { Politiche } \\
\text { Regionali }\end{array}$ & 96,9 & 95,3 & & 93,4 & & & 93,4 & & \\
Europee & & & 88,2 & & & 88,3 & & & 83,4 \\
\hline & 1992 & 1994 & 1995 & 1996 & 1999 & 2000 & 2001 & 2004 & \\
\hline $\begin{array}{l}\text { Politiche } \\
\text { Regionali }\end{array}$ & 91,1 & 91,2 & & 88,5 & & & 86,5 & & \\
Europee & & & 85,1 & & & 74,6 & & & \\
\hline
\end{tabular}

7 Tra i primi lavori ad occuparsi di questi temi, si veda il volume curato da M. CACIAGLI e P. CORBETTA, Elezioni regionali e sistema politico nazionale, Bologna, Il Mulino 1987. 
La crescita dell'astensionismo, in una regione storicamente "rossa" come la Toscana, mostra l'erosione e la crisi di un vecchio caposaldo della tradizionale cultura politica di questa regione: il voto come dovere civico e come momento espressivo di una forte e diffusa identità politico-ideologica. Qui si possono anche scorgere i segni della compiuta maturazione di una nuova logica di formazione del consenso politico: la crisi del cemento ideologico e l'allentamento dei vecchi collanti politico-culturali produce, in quote crescenti di cittadini, un approccio alla politica e alla scelta di voto che potremmo definire condizionato e selettivo, legato alla capacità che l'offerta politica, in quel momento e nelle diverse scadenze elettorali, mostra nel rappresentare interessi e forze sociali e nel motivare adeguatamente passioni e idee. All'interno di questo approccio, anche la scelta del non voto diviene un'opzione possibile e sentita come "legittima": un'opzione temporanea e revocabile, al mutare della proposta politica offerta all'elettore e delle condizioni in cui si svolge una specifica competizione elettorale.

Un'opzione revocabile, si noti bene: nulla di più erroneo di un approccio "catastrofista", che presuma di cogliere qui i segni di un qualche irreversibile declino della partecipazione politica, o di un incolmabile distacco tra società, politica e istituzioni. Ed infatti, con il terzo e più recente ciclo elettorale e soprattutto con le elezioni del 2004, la partecipazione elettorale in Toscana torna significativamente a crescere (quasi quattro punti), segnalando probabilmente un diverso clima politico e un più elevato livello di competitività delle consultazioni elettorali.

L'affermarsi e il diffondersi di queste nuove logiche nei comportamenti elettorali nasce certamente sullo sfondo dei grandi mutamenti che hanno investito le democrazie occidentali e la cultura politica diffusa, ma è stato indubbiamente anche aiutato e favorito da specifici fattori istituzionali, tra i quali in primo luogo i nuovi sistemi elettorali di tipo maggioritario, con i processi di personalizzazione della politica che ad essi si sono accompagnati. In particolare, in Italia, con l'elezione diretta dei sindaci, il voto amministrativo ha acquisito una più autonoma dimensione: scegliere un sindaco appare oramai, agli occhi di una buona parte dei cittadini, una scelta ben distinta dal voto politico con cui si elegge un deputato (o anche un candidato premier, sebbene nelle forme istituzionali un po' spurie con cui fino ad oggi ciò è accaduto). Sarebbe certo sbagliato sostenere che le identità politiche degli elettori non contino più (sia pure in modo diverso dal passato: oggi pesa sempre di più il senso di appartenenza ad un'area politica, più che ad un singolo partito), ma certo sono molti i segni (e ne vedremo molti nel corso di questa ricerca) che inducono a pensare come, specie nella sfera delle elezioni amministrative, la valutazione della specifica posta in gioco sia entrata oramai nella logica profonda delle scelte degli elettori. Insomma, quando si tratta di scegliere un sindaco, il giudizio sulle capacità dei candidati o sul lavoro svolto da un'amministrazione uscente, ha acquisito un rilievo crescente nelle motivazioni del voto, tale anche da trascendere, almeno in parte e sicuramente per una fetta notevole degli elettori, le appartenenze di schieramento politico, e tale da indurre comunque comportamenti elettorali differenziati, tra cui appunto una più accentuata "volatilità", o una forte oscillazione, nella stessa affluenza alle urne. 
Tra le motivazioni che accompagnarono la riforma del 1993, quelle che nascevano dalla preoccupazione sul calo della partecipazione erano certamente tra le più diffuse e condivise: eppure, come ora vedremo, il mutato sistema elettorale ed istituzionale, per un decennio, non ha frenato una tendenza alla crescita dell'astensionismo. Questa minore partecipazione elettorale alle elezioni amministrative, in generale e rispetto alle elezioni politiche, tuttavia, deve essere letta attraverso una pluralità di chiavi interpretative, che rifuggano da generiche imputazioni di responsabilità alla "crisi" dei partiti o della politica. Appare sempre più evidente, infatti, come dietro le percentuali del non voto si nascondano e si sommino fenomeni diversi: nel caso specifico del voto amministrativo, da una parte, in alcuni settori dell'elettorato, (quelli più apatici, meno interessati alla politica, o marginali socialmente e culturalmente), una minore mobilitazione può essere indotta dalla stessa specificità di tale competizione, dalla minore pressione ed esposizione mediatica, o dalla percezione della sua minore politicizzazione, del suo essere relativamente "meno importante", rispetto al voto politico (il che può contribuire a spiegare il perdurante divario tra voto politico e voto amministrativo); dall'altra parte, una scelta astensionista può nascere anche dalla risposta selettiva di un altro segmento dell'elettorato (in questo caso, all'opposto, quello più attento alla politica e più segnato da una propria identità politica) che, giudicando insoddisfacente l'offerta elettorale dello schieramento a cui pure ci si sente più vicini, e/o per altro verso giudicando ininfluente o non decisiva la propria partecipazione (ad esempio, quando si percepisce come scontato l'esito del voto), sceglie di non votare, attribuendo a questa opzione il valore di una scelta politica pienamente legittima e, talvolta, di una protesta o di un "dispetto".

Voto amministrativo e astensionismo in Toscana. - La Toscana si ritrova pienamente dentro questo quadro interpretativo fin qui delineato. Tendenze, forme e livelli della partecipazione elettorale mostrano come anche in Toscana si manifesti pienamente quel fenomeno che alcuni studiosi hanno definito come «astensionismo intermittente»: accanto ad un astensionismo «strutturale» che si fonda su una duratura e, per così dire, ormai congenita estraneità e indifferenza alla politica, emerge anche una crescente quota di elettori che scelgono di volta se e quando recarsi alle urne, secondo una mutevole valutazione delle proposte politiche in campo e dell'importanza attribuita alla posta in gioco.

A tutto ciò va aggiunto un altro fattore, che anche in Toscana ha giocato un ruolo rilevante: ossia, l'indebolimento dei partiti e delle loro strutture organizzative territoriali, molto meno forti e ramificate che in passato e quindi non più in grado di coinvolgere, e "trascinare" alle urne, anche i settori dell'elettorato meno motivati o più distanti dalla politica. Una delle cause non secondarie della minore partecipazione elettorale va individuata cioè nello stesso processo di destrutturazione dei partiti. E se un tale processo, in Toscana, appare certamente meno forte e ad uno stadio meno avanzato, rispetto ad altre aree del paese, esso tuttavia si è manifestato pienamente anche in questa regione. 
Naturalmente, per le elezioni amministrative, il radicamento territoriale dei partiti continua ad essere un fattore decisivo, così come la loro capacità di mettere a disposizione delle comunità locali un personale politico esperto e sperimentato, capace di sollecitare attenzione e attirare consenso; e tuttavia hanno pesato certamente, sui livelli più bassi di partecipazione elettorale, un indebolimento della capacità di presa dei partiti sulla società locale e una rarefazione del loro insediamento organizzativo. E la stessa figura del sindaco, se appare come il vero, nuovo baricentro della rappresentanza politica locale (argomento su cui ci soffermeremo ampiamente nel corso di questo lavoro e poi nelle conclusioni) non sembra però di per sé capace, evidentemente, di rappresentare l'elemento catalizzatore di quella più ampia partecipazione, che solo una politica "diffusa", la presenza di reti organizzative ramificate, assicurava in passato e può assicurare ancor oggi.

Questi fenomeni, (dapprima, per circa un decennio, una caduta del tasso di partecipazione e, insieme, un crescente divario tra il voto politico e quello amministrativo; e poi, con le ultime elezioni, una ripresa della partecipazione e una riduzione di quel divario) possono essere colti molto bene analizzando i dati relativi ai 51 Comuni toscani con oltre 15 mila abitanti, che costituiscono l'oggetto specifico della nostra indagine (FIG. 3).

FIG. 3. - Percentuale dei votanti nei comuni toscani con oltre 15 mila abitanti.

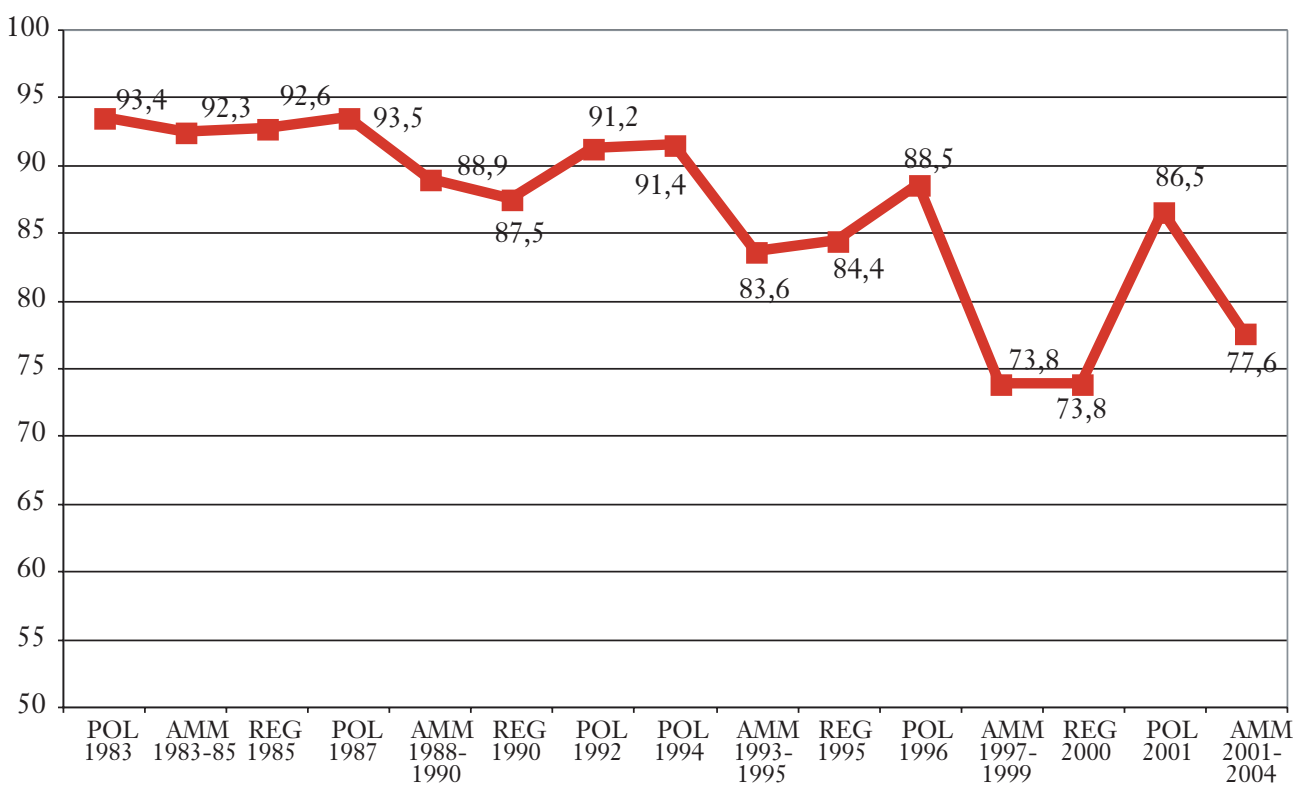

Come si può notare, ancora per quasi tutti gli anni Ottanta le percentuali dei votanti in Toscana, oltre che elevatissime, rimangono costanti, quale che sia il livello istituzionale a cui si riferiscono: il voto, dunque, quale che sia l'occasione elettorale, rimane ancora, pur in un decennio che comincia a far emergere i profondi mutamenti che stanno investendo i tradizionali pilastri di una cultura politica diffusa, il momento in cui esprimere comunque la propria identità e appartenenza politica. 
D'altra parte, il sistema proporzionale si fondava proprio su questo presupposto: un voto che non decideva direttamente su chi dovesse amministrare un comune, ma che era soprattutto espressione di un orientamento politico, un atto che costruiva una rappresentanza, ma che delegava però ai partiti, in una fase successiva e distinta, il compito di trovare gli accordi e le mediazioni per la definizione dell'assetto degli organi esecutivi. Soprattutto in una regione con un forte radicamento delle culture e delle tradizioni politiche, quale è stata la Toscana, ogni scadenza elettorale diventava un momento in cui era possibile (e necessario, vista l'importanza politica generale attribuita anche a piccoli spostamenti percentuali) manifestare la propria identità politica. Certo, sarebbe riduttivo pensare che, in passato, la specifica dimensione amministrativa non contasse, o che l'elettore non guardasse anche ai possibili effetti del proprio voto nella determinazione delle cariche elettive (i capilista, ad esempio, indicati o riconosciuti come possibili sindaci); ma è indubbio che pesassero, nello stesso "clima" elettorale del voto amministrativo, le grandi fratture politico-ideologiche che attraversavano il nostro paese.

Già con le elezioni amministrative che si svolsero tra il 1988 e il 1990, tuttavia, cresce notevolmente lo scarto con il voto politico: se nel 1983-1985, il divario era stato solo dell'1,1\%, nel ciclo elettorale 1987-1990 tale divario sale al 3,5\%, con una flessione piuttosto accentuata nei comuni con oltre 100 mila abitanti ($4,7 \%)$ e un po' inferiore nei comuni tra 15 e 30 mila abitanti $(-2,4 \%)$.

Sono i primi segni di una tendenza che assumerà negli anni seguenti un ritmo sempre più incalzante. Lo scenario politico di crisi della Prima Repubblica porta ad una ripresa della partecipazione alle politiche del 1992 e del 1994, ma già negli anni 1993-1995 il voto amministrativo (si noti bene: nonostante la novità della legge elettorale) vede accentuarsi la caduta dei tassi di partecipazione: nel complesso, rispetto al 1988-1990, il 5,3\% in meno, con una punta di $-8,0 \%$ nella città medie (tra 50 e 100 mila ab.). E cresce anche il divario rispetto alle politiche: se nel precedente ciclo elettorale, la differenza era stata di circa 4 punti e mezzo, le elezioni amministrative degli anni 1993-1995 segnano, rispetto alle politiche del 1994, uno scarto di quasi otto punti.

La successiva tornata di elezioni amministrative, negli anni 1997-1999, non inverte la tendenza: anzi, il divario rispetto al voto politico del 1996 cresce ulteriormente, giungendo a toccare quasi i 15 punti percentuali; mentre lo stesso confronto con le precedenti elezioni amministrative segna un'ulteriore flessione di circa 10 punti. Va poi rilevata una particolarità: nell'arco di tempo considerato, le elezioni regionali si sono svolte in Toscana nella stessa data dei turni amministrativi generali, che coinvolgevano gran parte dei comuni toscani $(1985,1990,1995)$ : e ovviamente, il livello di partecipazione era pressoché identico ${ }^{8}$. Nel 2000, per la prima volta le elezioni regionali si svolgono separatamente, ma il tasso di partecipazione continua

8 Le percentuali dei votanti alle Regionali, nei nostri dati, si differenziano leggermente dal contemporaneo ciclo elettorale amministrativo in quanto, come già ricordato, abbiamo considerato unitariamente, nei diversi cicli elettorali, i Comuni che votavano al di fuori della tornata amministrativa principale. 
ad allinearsi a quello delle precedenti elezioni amministrative, assestandosi al livello più basso mai registratosi nella storia elettorale della Toscana: a segnalare, probabilmente, come, nella percezione dell'opinione pubblica, l'istituzione regionale fosse ancora tendenzialmente assimilata ad altri livelli amministrativi, e non evocasse una mobilitazione elettorale tipica delle scadenze politiche. Ed infatti, nelle successive elezioni politiche del 2001, la partecipazione tornava a crescere di oltre 12 punti: tra il 73,8\% delle amministrative del 1997-1999 e delle regionali del 2000, e l'86,5\% delle politiche 2001, si misurava il più forte scarto storicamente mai registratosi in Toscana tra elezioni svoltesi a distanza così ravvicinata.

L'ultimo ciclo elettorale, 2000-2004 segna, come abbiamo detto, un'inversione di tendenza: nei 51 comuni considerati, la percentuale dei votanti alle amministrative torna a crescere, per la prima volta dopo oltre vent'anni, passando dal $73,8 \%$ al $77,6 \%$ e riducendosi nettamente anche lo scarto rispetto alle precedenti politiche del 2001. Non si può non mettere in relazione questo dato al particolare clima politico che ha caratterizzato questa prima parte del decennio, con la crescita della mobilitazione politica indotta dall'opposizione al governo Berlusconi e dalle numerose forme di partecipazione dal basso con cui questa si è espressa. Ed è possibile poi ipotizzare che in Toscana sia tornata ad alimentarsi, almeno in parte, anche quella peculiare forma di identità politica municipalistica, o anche quella forma di "orgoglio" regionalistico, in opposizione agli equilibri politici nazionali, che da sempre costituiscono un tratto costitutivo della cultura politica della sinistra in questa regione. In ogni caso, emerge una conferma di quanto "mobili" siano divenuti, specie in occasione di elezioni amministrative, le forme e i livelli della partecipazione elettorale: una mobilità che certamente rappresenta una netta soluzione di continuità, rispetto ad un'immagine classica della Toscana "rossa" come regione dal forte e compatto profilo sub-culturale.

Partecipazione e astensionismo aggiuntivo nei ballottaggi. - Per completare il quadro, occorre anche considerare anche la partecipazione elettorale nei ballottaggi (TAB. 2): come vedremo meglio in seguito, analizzando il fenomeno da un altro punto di vista, in Toscana il ricorso ai ballottaggi è stato piuttosto frequente nel primo ciclo elettorale (19 casi su 51 comuni), mentre si è nettamente ridotto nel secondo e nel terzo ciclo elettorale (solo 8 e 10 casi), a causa della maggiore ampiezza delle coalizioni e della maggiore polarizzazione della competizione.

TAB. 2. - Toscana. Percentuale dei votanti alle elezioni politiche, regionali ed europee.

\begin{tabular}{|c|c|c|c|c|c|c|c|}
\hline \multirow{2}{*}{$\begin{array}{c}\begin{array}{c}\text { Ciclo } \\
\text { elettorale }\end{array} \\
1993-1995\end{array}$} & \multirow{2}{*}{$\begin{array}{c}\begin{array}{c}\text { Totale } \\
\text { comuni }\end{array} \\
51\end{array}$} & \multirow{2}{*}{$\begin{array}{c}\text { Comuni con } \\
\text { elezione al } \\
1^{\circ} \text { turno }\end{array}$} & \multicolumn{2}{|c|}{ Comuni al ballottaggio } & \multirow{2}{*}{$\begin{array}{c}\begin{array}{c}\% \text { votanti } \\
1^{\circ} \text { turno }\end{array} \\
81,6\end{array}$} & \multirow{2}{*}{$\begin{array}{c}\begin{array}{c}\% \text { votanti } \\
\text { ballottaggio }\end{array} \\
67,2\end{array}$} & \multirow{2}{*}{$\begin{array}{c}\begin{array}{c}\text { Astensionismo } \\
\text { aggiuntivo } \\
(\%)\end{array} \\
14,4\end{array}$} \\
\hline & & & 19 & totale & & & \\
\hline & & & & media & 82,9 & 70,0 & 12,9 \\
\hline \multirow[t]{2}{*}{ 1997-1999 } & 51 & 43 & 8 & totale & 71,4 & 56,8 & 14,6 \\
\hline & & & & media & 73,7 & 58,6 & 15,1 \\
\hline \multirow[t]{2}{*}{ 2000-2004 } & 52 & 42 & 10 & totale & 75,1 & 57,8 & 17,3 \\
\hline & & & & media & 75,2 & 61.4 & 13,8 \\
\hline
\end{tabular}


In Toscana, le dimensioni dell'astensionismo aggiuntivo (ossia, la percentuale di elettori in più che non vanno a votare, rispetto agli astenuti del primo turno) appaiono molto simili, per quanto riguarda il primo ciclo elettorale, a quelle riscontrate sul piano nazionale, pari al $13 \%$ degli elettori ${ }^{9}$ : nei 19 ballottaggi degli anni 1993-1995 svoltisi in Toscana abbiamo una flessione media del 12,9\% dei votanti, rispetto al primo turno, che sale fino al 15,1\% negli 8 ballottaggi del 1997-1999.

Il terzo ciclo elettorale vede una media di astensionismo aggiuntivo che torna a scendere $(13,8 \%)$, con una maggiore divaricazione rispetto al dato totale relativo al complesso degli elettori (17,3\%), a causa della particolare incidenza del ballottaggio nel Comune di Firenze, dove la partecipazione è scesa notevolmente, dal $76 \%$ al $51,9 \%$.

Le dimensioni del fenomeno appaiono, nel complesso, del tutto fisiologiche, (a dispetto dei detrattori del doppio turno che, puntualmente, cercano di trovare qui qualche argomento a favore delle proprie tesi): è evidente infatti come, in occasione dei ballottaggi, le motivazioni che possono spingere alla partecipazione sono diverse e mutevoli e, in alcuni casi, decisamente più deboli. Per un verso, una parte degli elettori, quanti avevano scelto al primo turno candidati esclusi dal ballottaggio, trova evidentemente minori motivazioni per tornare alle urne, se non quelle legate alla possibilità di esprimere comunque una seconda scelta, o di scegliere il "meno peggio" tra i due candidati rimasti in corsa; ma poi, soprattutto, per altro verso, incide una variabile decisiva (che, ad esempio, ha contribuito non poco ad abbassare il numero dei votanti nel ballottaggio di una città come Firenze, nel 2004), ossia il diverso grado di apertura o chiusura della competizione: tanto più incerto appare l'esito del ballottaggio, tanto maggiori sono le sollecitazioni (per tutti gli elettori, compresi quelli degli "altri" candidati) che possono indurre a partecipare al ballottaggio. Perciò, appare opportuno aggiungere alla considerazione dell'astensionismo aggiuntivo anche la valutazione di altre due misure: la chiusura della competizione, appunto, e quella che è stata definita la «partecipazione inattesa», cioè la quota di elettori che hanno votato al ballottaggio e che supera la quota dei voti raccolti dai primi due candidati ${ }^{10}$. Dal com-

9 Cfr. G. BALDINI e G. LEGNANTE, Città al voto, cit., p.157.

${ }^{10}$ Ivi, pp.157-158: la «partecipazione inattesa» è data dalla «differenza fra la partecipazione al ballottaggio e la quota di voti ottenuta al primo turno dai due candidati ammessi al ballottaggio». Tale differenza va calcolata sul totale degli elettori. Ad esempio, tra i comuni toscani, si prenda il caso del ballottaggio con il più alto valore di tale indice, le elezioni di Pietrasanta nel 1993: al primo turno, su 21.239 aventi diritto, avevano votato 17.395 elettori (81,9\%); al ballottaggio, 15.101 (71,1\%); il $1^{\circ}$ candidato ottenne 4.674 voti, il $2^{\circ}$ candidato 3.027 voti, tutti gli altri candidati esclusi dal ballottaggio ben 7.902 voti. A questo punto, va calcolata la differenza tra il numero dei votanti al ballottaggio e la somma dei voti raccolti dai primi due candidati: nel nostro caso, 15.101-(4.674+3.027)=7.400 che corrispondono al 34,8\% degli elettori. Otteniamo così una misura della quota di elettori che, pur (teoricamente) "disinteressati", in quanto avevano scelto al primo turno candidati poi esclusi, hanno partecipato ugualmente al ballottaggio. Nel nostro caso si tratta di un valore molto elevato, che possiamo confrontare con quello dell'astensionismo aggiuntivo (10.8\%): se ne può trarre la conclusione che, a fronte di 10 elettori che si sono astenuti al ballottaggio, dopo aver votato al primo turno, ve ne sono stati ben 35 che, pur essendo "orfani" del proprio candidato, sono tornati alle urne. 
binarsi di queste diverse variabili, possono derivare situazioni diverse, che possono avere ripercussioni diverse sui livelli di mobilitazione degli elettori al secondo turno: da una parte, ballottaggi molto serrati e incerti o, al contrario, dall'esito piuttosto scontato; dall'altra, una quota più o meno elevata di elettori (tra quelli che avevano votato per gli "altri") che rientra in gioco al secondo turno, ridistribuendo il proprio voto tra i due candidati rimasti in campo. Per misurare il grado di "chiusura" della competizione occorre considerare non solo il distacco tra il primo e il secondo candidato, ma anche la loro distanza dalla soglia del 50\% (e quindi di converso, la quantità di "altri" potenziali voti a cui è possibile attingere $)^{11}$. Nelle tabelle seguenti presentiamo il quadro dei ballottaggi svoltisi in Toscana, distinguendo i casi di ballottaggio per ciclo elettorale (TAB. 3) e per classi di chiusura della competizione (TAB. 4).

TAB. 3. - Astensionismo aggiuntivo e partecipazione inattesa nei ballottaggi.

\begin{tabular}{ccccccc}
\hline $\begin{array}{c}\text { Ciclo } \\
\text { elettorale }\end{array}$ & $\begin{array}{c}\text { Numero } \\
\text { di casi }\end{array}$ & & $\begin{array}{c}\text { Astensionismo } \\
\text { al } 1^{\circ} \text { turno }\end{array}$ & $\begin{array}{c}\text { Astensionismo } \\
\text { aggiuntivo* }\end{array}$ & $\begin{array}{c}\text { Partecipazione } \\
\text { inattesa** }\end{array}$ & $\begin{array}{c}\text { Indice di } \\
\text { chiusura della } \\
\text { competizione }\end{array}$ \\
\hline $1993-1995$ & 19 & totale & 18,4 & 14,4 & 14,9 & 2,9 \\
$1997-1999$ & 8 & totale & 28,6 & 14,6 & 3,6 & 6,3 \\
$2000-2004$ & 10 & media & 26,3 & 15,1 & 6,1 & \\
& & totale & 24,9 & 17,3 & $-0,4$ & 5,2 \\
TOTALE & 37 & totale & 22,9 & 13,8 & 3,0 & 4,3 \\
& & media & 21,2 & 13,6 & 7,1 & \\
\hline
\end{tabular}

*Astensionismo aggiuntivo = differenza fra la quota di astenuti al ballottaggio e quella degli astenuti al primo turno.

** Partecipazione inattesa $=$ differenza fra la partecipazione al ballottaggio e la quota di voti ottenuta al primo turno dai due candidati ammessi al ballottaggio.

${ }^{11}$ L'indice di «chiusura della competizione» (IC), quindi, si costruisce calcolando «la differenza fra la distanza fra il primo e il secondo candidato e la distanza del primo classificato dal $50 \%$, calcolata in \% sui voti validi» (G. BALDINI e G. LEGNANTE, Città al voto, cit., p. 158). Ossia, secondo questa formula: $\mathrm{IC}=\left(\%\right.$ voti $1^{\circ}$ candidato $-\%$ voti $2^{\circ}$ candidato $)-\left(50 \%-\%\right.$ voti $1^{\circ}$ candidato $) . \mathrm{Si}$ possono così ottenere varie classi di chiusura della competizione: nella tabella 3 , la prima di esse ("meno - 10\%") indica una competizione molto aperta, e così via, fino all'estremo opposto ("più di $10 \%$ "), dove la competizione è invece molto chiusa. Il caso di ballottaggio più «aperto», tra quelli svoltisi in Toscana, è quello delle elezioni di Lucca nel 1994, con il $1^{\circ}$ candidato al 30,7\% e il secondo al 30,4 (IC = -19,0). Il ballottaggio più «chiuso», invece, quello di Pescia, nel 1997, con il $1^{\circ}$ candidato al 46,3\% e il secondo al 16,5\% (IC=26,1). Il ballottaggio di Firenze, nel 2004, ha visto un IC molto elevato, (pari a 18,6 ), con il sindaco uscente al $1^{\circ}$ turno al $49,2 \%$ e il suo principale contendente al $29,7 \%$. 
TAB. 4. - Astensionismo aggiuntivo e partecipazione inattesa nei ballottaggi, per classi di chiusura della competizione.

\begin{tabular}{lcccccc}
\hline Ballottaggi & $\begin{array}{c}\text { Indice di } \\
\text { chiusura della } \\
\text { competizione* }\end{array}$ & $\begin{array}{c}\text { Numero } \\
\text { di casi }\end{array}$ & & $\begin{array}{c}\text { Astensionismo } \\
\text { aggiuntivo al } \\
\text { primo turno }\end{array}$ & $\begin{array}{c}\text { Astensionismo } \\
\text { aggiuntivo al } \\
2^{\circ} \text { turno }\end{array}$ & $\begin{array}{c}\text { Partecipazione } \\
\text { inattesa }\end{array}$ \\
\hline $\begin{array}{l}\text { "molto } \\
\text { aperti" }\end{array}$ & Meno di -10\% & 5 & Media & 20,6 & 12,4 & 21,5 \\
"aperti" & Da -10\% a 0\% & 8 & Media & 20,2 & 10,5 & 12,4 \\
"chiusi" & Da 0\% a 10\% & 6 & Media & 24,8 & 15,8 & 10,0 \\
"molto & Più di + 10\% & 12 & Media & 20,8 & 15,1 & 8,2 \\
chiusi" & TOTALE & 37 & Media & 21.2 & 13,6 & 11,5 \\
\hline
\end{tabular}

* Chiusura della competizione = differenza fra la distanza fra il primo e il secondo classificato e la distanza del primo classificato dal $50 \%$, calcolata sui voti validi.

La partecipazione inattesa appare piuttosto alta nei ballottaggi del primo ciclo elettorale (quello, peraltro, con il maggior numero di casi), segnato da una minore concentrazione bipolare e da una più elevata "apertura" della competizione: mediamente, oltre il $18 \%$ di elettori, con un astensionismo aggiuntivo del $12.9 \%{ }^{12}$. Negli otto ballottaggi degli anni 1997-1999 questa percentuale si abbassa notevolmente, fino ad una media del $6,1 \%$ (ma un dato totale ancora più basso, il 3,6\%), a fronte di un astensionismo aggiuntivo più elevato $(15,1 \%)$. Infine, nei 10 ballottaggi del 2000-2004 il dato di partecipazione inattesa si abbassa notevolmente: con una media del $3,0 \%$ e con un valore negativo relativo al totale degli elettori $(-0,4 \%)$, dovuto al fatto che in quattro comuni su 10 (e tra questi Firenze) gli elettori che hanno votato al $2^{\circ}$ turno sono stati in numero inferiore anche rispetto a quelli che, al primo turno, avevano votato per gli stessi due candidati giunti al ballottaggio.

Considerando nel loro insieme i 37 casi di ballottaggio che si sono avuti in Toscana a partire dal 1993, appare nettamente una correlazione tra il livello di partecipazione elettorale e la "chiusura" o "apertura" della competizione.

Più aperta la competizione, ossia più incerto il suo esito, più elevato il grado di coinvolgimento degli elettori che, al primo turno, avevano votato per gli "altri" candidati: la percentuale di "partecipazione inattesa" passa da una media del $21,5 \%$ nei 5 ballottaggi molto aperti, al 12,4\% nei ballottaggi aperti, al 10,0\% nei casi di ballottaggi chiusi e all' $8,2 \%$ in quelli molto chiusi.

12 Nella tabella distinguiamo tra media e totale, per consentire una prima valutazione differenziata tra ciò che accade mediamente nei diversi casi e ciò che invece riguarda il comportamento degli elettori, nel loro complesso. Ovviamente, quando i due valori sono vicini se ne può dedurre una relativa omogeneità dei fenomeni in tutti i comuni, quale che sia la loro dimensione demografica. 
I voti non validi. - Infine, particolare attenzione merita anche un'altra modalità di voto, ossia il voto non valido, le schede bianche e le schede nulle. È opportuno distinguere tra i due tipi di voto non valido, in quanto le schede bianche rappresentano con nettezza un'espressione di rifiuto dell'offerta elettorale, mentre lo schede nulle possono contenere questa motivazione, ma possono anche essere il frutto di errori materiali nell'espressione del voto: un aspetto che occorre particolarmente considerare quando si tratta, come nel nostro caso, di valutare gli effetti di un nuovo sistema elettorale sulle forme e i livelli della partecipazione. Come mostra la FIG. 4, nella vicenda delle elezioni amministrative in Toscana uno spartiacque fondamentale si rivela il ciclo elettorale degli anni 1988-1990: come già per la percentuale dei votanti (che vede allora la prima brusca flessione dell'intera storia elettorale della regione, con una flessione del 3,4\%), anche il voto non valido registra allora un incremento significativo, passando le schede bianche (nei 51 Comuni che stiamo analizzando) dal 2,4\% al 2,8\% e le nulle dal 2,2\% al 3,3\%. Si tratta, con tutta evidenza, di un fenomeno da ricondurre al particolare clima politico e culturale di quel biennio a cavallo del fatidico Ottantanove e, in particolare, ai possibili fattori di disorientamento e di incertezza che, specie in una regione come la Toscana, potevano essere legati agli eventi di portata epocale che in quel momento maturavano.

FIG. 4. - Percentuale di schede bianche e nulle (sui votanti) alle elezioni comunali, nei comuni toscani con oltre 15 mila abitanti.

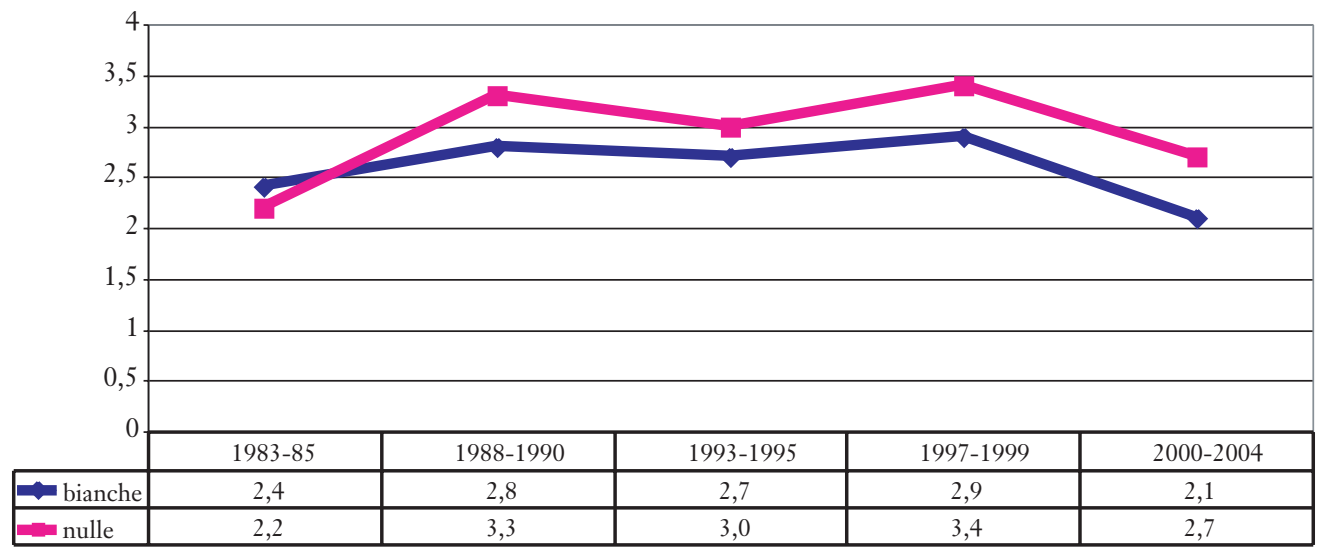

Meno rilevante appare invece un'interpretazione che rimanda alle difficoltà informative legate all'introduzione di un nuovo sistema elettorale: alla prima prova della nuova legge elettorale, nel biennio 1993-1995, decrescono leggermente sia la percentuale delle schede bianche $(-0,1 \%)$ sia quella delle schede nulle $(-0,3 \%)$; mentre, alla seconda prova, e dunque quando si poteva pensare che fosse più avanzato un processo di apprendimento delle nuove regole, crescono nuovamente sia le schede bianche $(+0,2 \%)$ che le schede nulle $(+0,4 \%)$. Infine, con il 
terzo ciclo elettorale, tra il 2000 e il 2004, emerge una nuova, e piuttosto netta, inversione di tendenza: le schede bianche, dal 2,9\% registrato nel ciclo 1997-1999 diminuiscono fino al 2,1\%, mentre le schede nulle passano dal 3,4\% al 2,7\%, con una flessione di notevole entità. Se consideriamo anche la ripresa della percentuale dei votanti, sopra ricordata $(+1,5 \%)$, otteniamo una significativa riduzione dell'area del non voto, di circa 3 punti: un segnale piuttosto netto di una nuova fase di ripresa della partecipazione politica ed elettorale.

\section{Nuove regole e nuove modalità di partecipazione}

Maggioritario e astensionismo: quale legame? - Quale dunque il bilancio che è possibile trarre da questo primo punto di osservazione? La nuova legge elettorale, l'elezione diretta del sindaco, quali effetti hanno prodotto sulle forme e i livelli della partecipazione elettorale?

Come dicevamo, nel dibattito che ha accompagnato la riforma del 1993, questo tema era molto presente: da più parti si sosteneva la tesi secondo cui le forme di elezione personalizzata (i "nuovi" sindaci, i collegi uninominali) avrebbero consentito un più diretto e ampio rapporto tra la politica e i cittadini. Inoltre, la polemica contro la cosiddetta partitocrazia si alimentava anche di questa convinzione: ossia, che la caduta della partecipazione elettorale, il crescente astensionismo, sarebbero stati frenati dall'attribuzione ai cittadini di un più diretto potere decisionale, creando le condizioni affinché essi potessero influire in prima persona sulla scelta degli uomini e delle donne che li avrebbero governati o amministrati. Come si è visto, ciò non è accaduto; ma non per questo, naturalmente, se ne può trarre la conclusione di un qualche fallimento delle nuove regole. Sono altre, con tutta evidenza, le ragioni di fondo che tendono oramai a rendere l'astensione una componente strutturale del comportamento elettorale: una componente peraltro assai variabile, tanto nelle dimensioni (come si è appena visto, anche nel caso toscano), quanto nelle motivazioni che ad essa risultano sottese. Ragioni profonde, che rimandano, sullo sfondo, al travaglio della crisi e della "transizione" italiana degli anni Novanta: certamente, nel vecchio sistema politico italiano, la logica della rappresentanza proporzionale e la presenza di forti e strutturati partiti di massa contribuiva a tenere alto il livello della mobilitazione politica e quello della partecipazione elettorale. Oggi, con un'imperfetta democrazia maggioritaria e, soprattutto, con partiti e coalizioni fragili, che riescono ad intessere solo legami deboli con la società e i cittadini, - e d'altra parte, una società civile, specie in una regione come la Toscana, ricca di un tessuto associativo autonomo e pluralistico -, il momento della partecipazione elettorale amministrativa (laddove si tratta di scegliere "solo" un sindaco, beninteso: perché, per le elezioni politiche, molte linee di frattura politica e ideologica continuano a pesare, e non marginalmente) si è parzialmente depotenziato politicamente: si stanno affievolendo cioè quei caratteri di permanente 
scelta di campo che, in altre epoche, accompagnava anche questi livelli di competizione elettorale. Come hanno mostrato le numerose analisi sulle elezioni della "transizione" italiana, l'elettorato italiano è stato caratterizzato, nel complesso e almeno fino alle elezioni del 2001, da una grande stabilità e una scarsa permeabilità reciproca tra i due blocchi politici, a fronte di una notevole mobilità nel voto ai partiti all'interno delle coalizioni: e l'esito delle elezioni politiche del 1994, del 1996 e del 2001, com'è noto, è stato soprattutto determinato dalla diversa configurazione dell'offerta politica, dall'assetto delle coalizioni e delle alleanze elettorali.

Una tale valutazione vale solo in parte per le elezioni amministrative: sarebbero necessarie ricerche specifiche, per valutare la maggiore o minore mobilità del voto tra gli schieramenti, in occasione di elezioni comunali, ma si può facilmente ipotizzare (anche alla luce dei numerosi casi in cui città chiaramente orientate nel voto politico hanno scelto sindaci di altro colore) che essa sia certamente qui più elevata. Tuttavia, proprio i dati sulla partecipazione elettorale, ci inducono a sottolineare come sia soprattutto quest'ultima variabile (ossia, di volta in volta, il grado diverso di mobilitazione dell'elettorato dei due maggiori schieramenti) ad incidere in maniera decisiva sullo stesso esito elettorale: se, in sede di elezioni politiche, il senso di appartenenza ad uno dei due poli è molto radicato e (fino ad oggi) raramente è accaduto che esso venisse superato con un'aperta e diretta "defezione" verso l'altro polo, in occasione di elezioni amministrative, invece, votare per un "altro" candidato sindaco, diverso da quello proposto dallo schieramento a cui ci si sente politicamente più vicini, è una scelta che viene percepita (da una parte almeno degli elettori) come possibile e "legittima", in quanto foriera di effetti politici comunque circoscritti ${ }^{13}$. Ma soprattutto, ci sembra di poter ipotizzare, è lo scegliere di non votare la soluzione a cui più frequentemente ricorre quella fetta di elettori che non si riconosce nella proposta dello schieramento politico a cui ci si sente abitualmente più vicini. Per riprendere le note categorie di Hirschmann, l'astensione può essere vista come una forma di voice: e non a caso, nel dibattito corrente, il non voto è spesso letto come "protesta", come espressione di un voto di dissenso (ma anche di una "punizione", di un voto "a dispetto") verso le scelte del proprio schieramento, che non si spinge tuttavia fino al passo estremo, ossia alla defezione verso l'altro campo politico.

\footnotetext{
${ }^{13}$ Anche per questo sono pienamente condivisibili tutte le valutazioni che, in occasione di elezioni amministrative, raccomandano molta prudenza nel trarre indicazioni politiche generali dagli esiti del voto. Da ultimo, si veda, sulle elezioni del 2004, e su questo tema, quanto scrive G. LEGNANTE sul «ruolo delle elezioni locali nel "ciclo" elettorale nazionale» in Il voto nelle città: ancora una sconfitta per il centrodestra, in Politica in Italia, edizione 2005, a cura di C. Guarnieri e J.L. Newell, Bologna, Il Mulino 2005, pp. 81-100.
} 
Questa dinamica dei comportamenti elettorali, nelle elezioni comunali, trova un terreno particolarmente favorevole in occasione dei ballottaggi: è qui che spesso si manifesta la scelta dell'astensione come espressione di un rifiuto della proposta politica che è stata sottoposta agli elettori ${ }^{14}$.

Nel valutare poi i fenomeni dell'astensionismo e del non voto, occorre anche considerare un altro argomento, sostenuto in modo ricorrente dagli avversari dei modelli maggioritari, secondo cui tali modelli, restringendo di fatto l'area e il ruolo della rappresentanza, scoraggerebbero una più ampia partecipazione: gli elettori che non si riconoscono nella logica bipolare, ad esempio, sarebbero indotti con maggiore frequenza all'astensione. Ebbene, come si ricordava sopra, se non si può escludere che un atteggiamento di "rifiuto" dell'offerta elettorale possa essere alla base di una certa quota di astensionismo differenziale e intermittente, $\mathrm{i}$ dati disponibili ci suggeriscono che non vi è alcuna correlazione tra ampiezza dell'offerta di rappresentanza politica e decremento dell'astensionismo: anzi, specie per i primi due cicli elettorali, si nota il fenomeno esattamente inverso. Se guardiamo alla percentuale di aumento dei non votanti, nelle elezioni amministrative, rispetto alle più vicine elezioni politiche, notiamo che, nei comuni in cui più alto è il numero delle liste concorrenti e dei candidati sindaci, maggiore è anche l'incremento dell'astensionismo (FIGG. 5a, 5b, 6a e 6b): ciò accade, in Toscana, in modo evidente sia per le elezioni comunali degli anni 1993-1995, rispetto al voto politico del 1994, sia per le elezioni degli anni 1997-1999, rispetto alle politiche del 1996. Una tendenza ben presente, peraltro, anche su scala nazionale, come notavano Baldini e Legnante: «gli elettori sembrano più disorientati dalla frammentazione della contesa elettorale che non dalla sua semplificazione» ${ }^{15}$. Meno lineare la correlazione per le elezioni comunali del 2000-2004, rispetto alle politiche del 2001 (FIGG. 7a e 7b), soprattutto per quanto riguarda i candidati, anche perché, come vedremo meglio tra poco, tende a diminuire, in quest'ultimo ciclo elettorale, l'offerta di candidati sindaci, mentre continua a crescere quella delle liste. E pur tuttavia, anche per quest'ultimo ciclo elettorale, se non possiamo dedurre che la causa dell'astensionismo sia di per sé la maggiore frammentazione

${ }^{14} \mathrm{Il}$ peso di un'astensione aggiuntiva differenziata nel determinare l'esito dei ballottaggi è fenomeno evidente e diffuso: uno dei casi più clamorosi è quello delle elezioni comunali di Bologna nel 1999, studiato nel volume di G. BALDINI, P.CORBETTA e S. VASSALlO, La sconfitta inattesa, Bologna, Il Mulino 2000 In particolare (pp. 28-35), come notano questi studiosi, l'astensionismo aggiuntivo, verificatosi in occasione del ballottaggio, risultava nettamente caratterizzato socialmente e politicamente, avendo riguardato le aree della città più popolari e di tradizionale insediamento del Pci-Pds. In generale, e guardando anche ai casi toscani, la crescita delle astensioni, al secondo turno, nei casi dei ballottaggi più "aperti", si può attribuire alla scarsa coesione tra gli elettori delle diverse forze politiche pure appartenenti allo stesso schieramento e/o alla debole capacità dei candidati a conservare tutto il potenziale bacino di consensi che si erano espressi al primo turno. In Toscana, quest'ultima appare una possibile spiegazione dei casi di ballottaggio in cui si rovesciano i rapporti di forza delle elezioni politiche (ad esempio, a Lucca nel 1994, a vantaggio del centrosinistra) o lo stesso esito del primo turno (come ad Arezzo, sia nel 1999 che nel 2004, a vantaggio del centrodestra).

${ }^{15}$ G. BALdini e G. LEgNANTE, Città al voto, cit., pp. 135-136. 
dell'offerta, possiamo però concludere che l'allargamento dell'offerta non ha certamente contribuito a ridurre, in termini relativi, lo scarto rispetto al voto politico.

FIG. 5a. - Numero delle liste concorrenti alle elezioni amministrative (1993-95) e incremento dell'astensionismo rispetto alle elezioni politiche del 1994. Comuni con oltre 15mila abitanti.

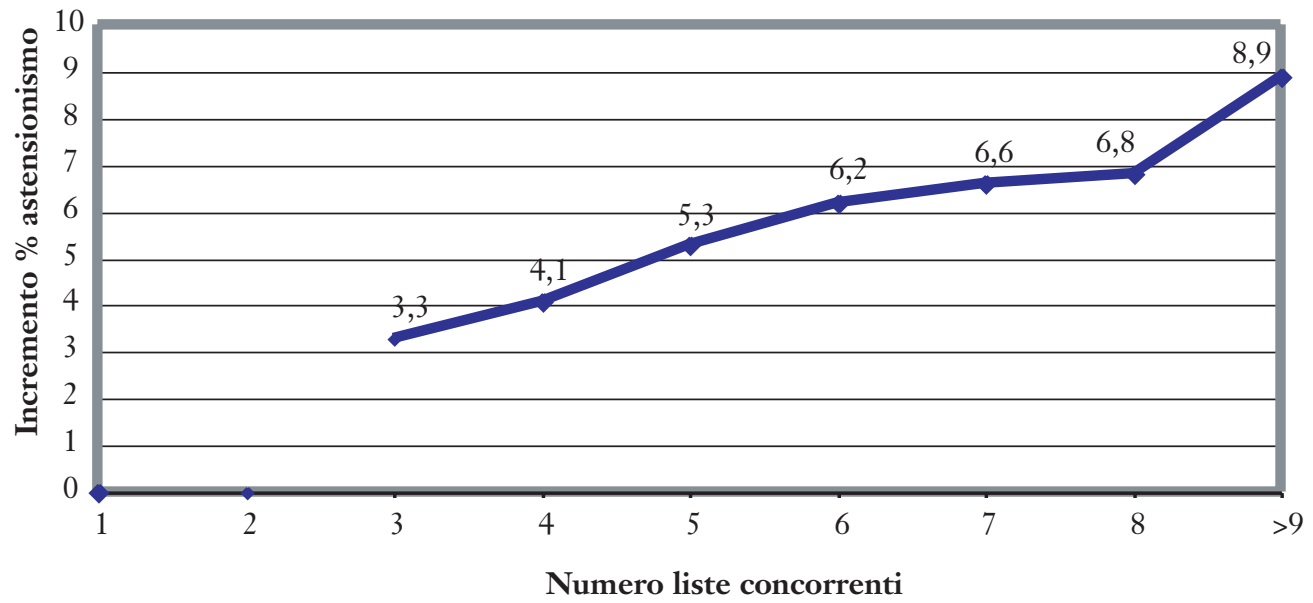

FIG. 5b. - Toscana. Numero dei candidati alle elezioni amministrative (1993-95) e incremento dell'astensionismo rispetto alle elezioni politiche del 1994. Comuni con oltre 15mila abitanti.

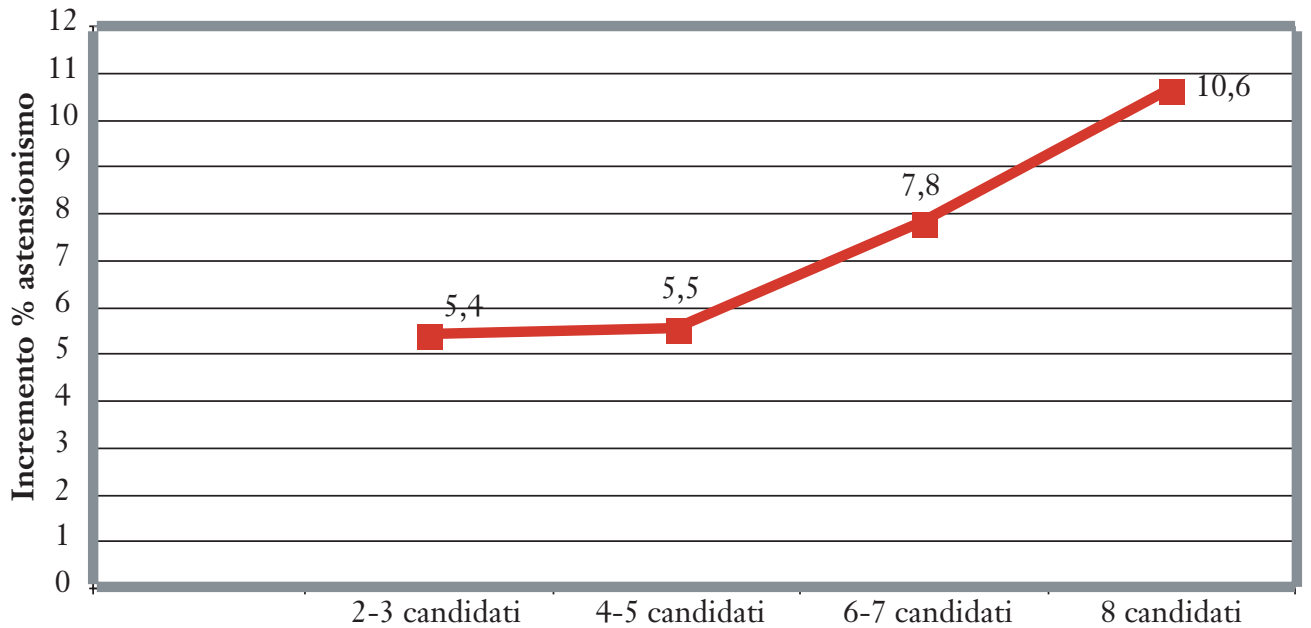


FIG. 6a. - Toscana. Numero delle liste concorrenti alle elezioni amministrative (1997-99) e incremento dell'astensionismo rispetto alle elezioni politiche del 1996. Comuni con oltre 15 mila abitanti.

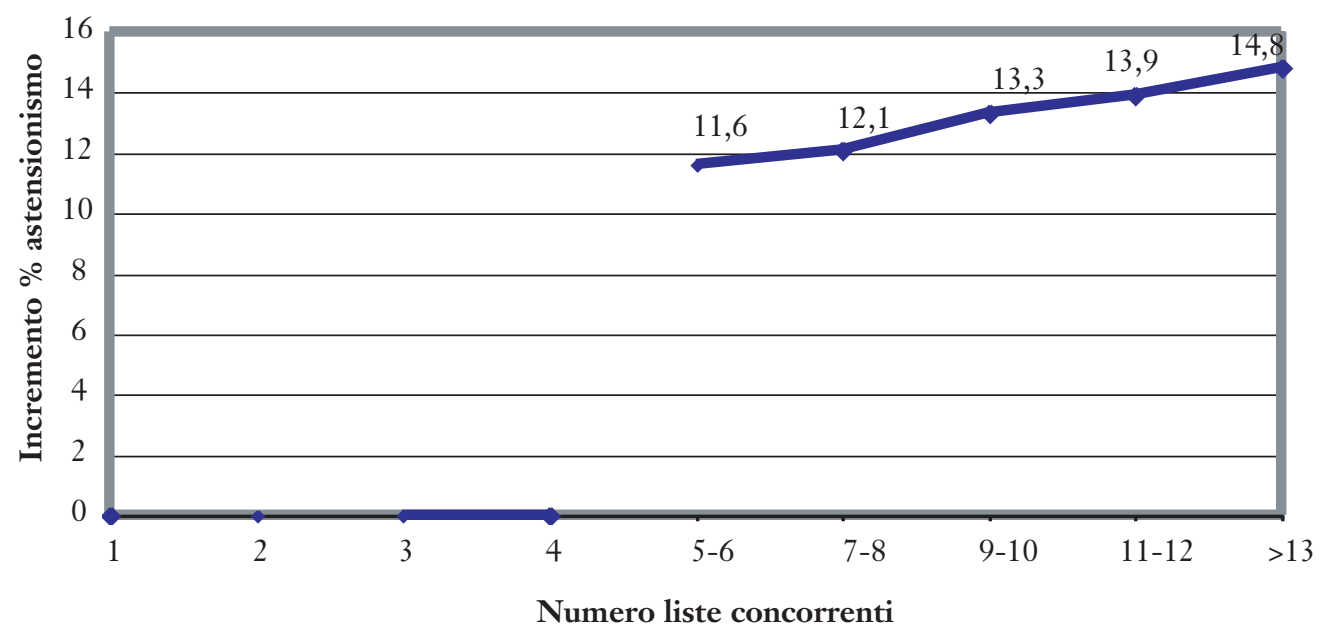

FIG. 6b. - Toscana. Numero dei candidati alle elezioni amministrative (1997-99) e incremento dell'astensionismo rispetto alle elezioni politiche del 1996. Comuni con oltre 15mila abitanti.

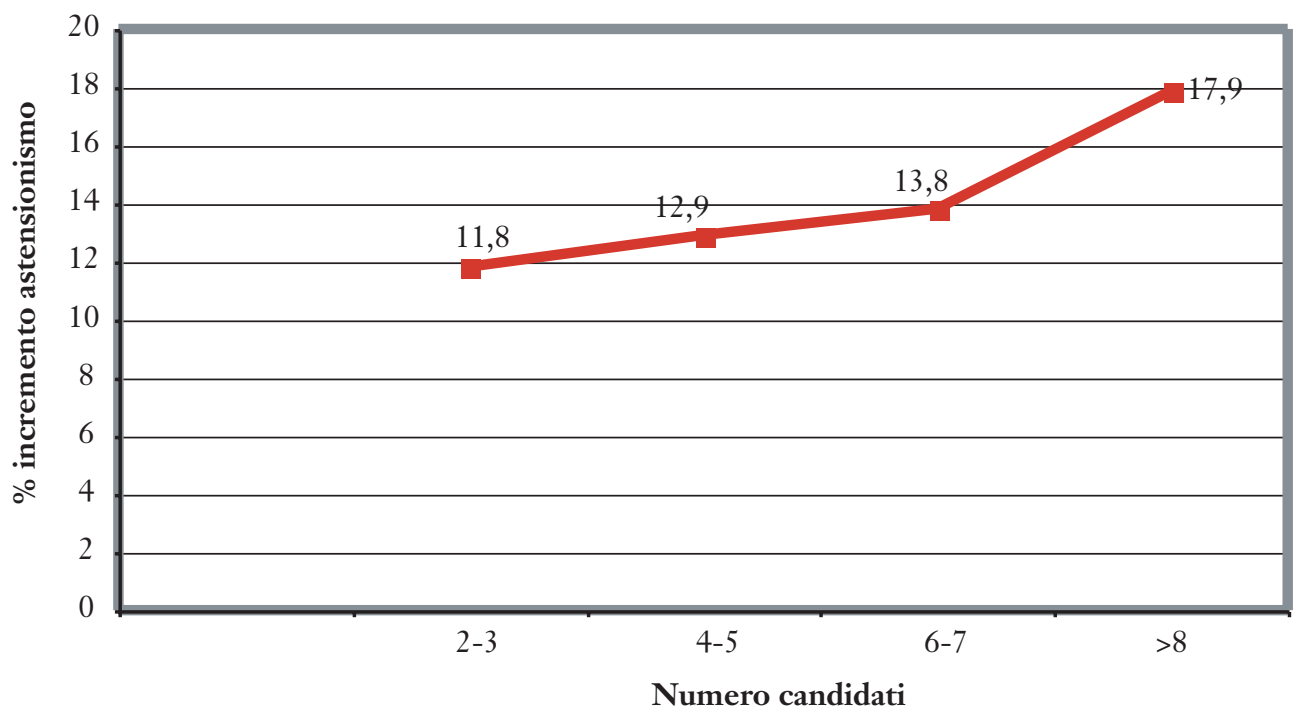


FIG. 7a. - Toscana. Numero delle liste concorrenti alle elezioni amministrative (2000-2004) e incremento dell'astensionismo rispetto alle elezioni politiche del 2001. Comuni con oltre 15 mila abitanti.

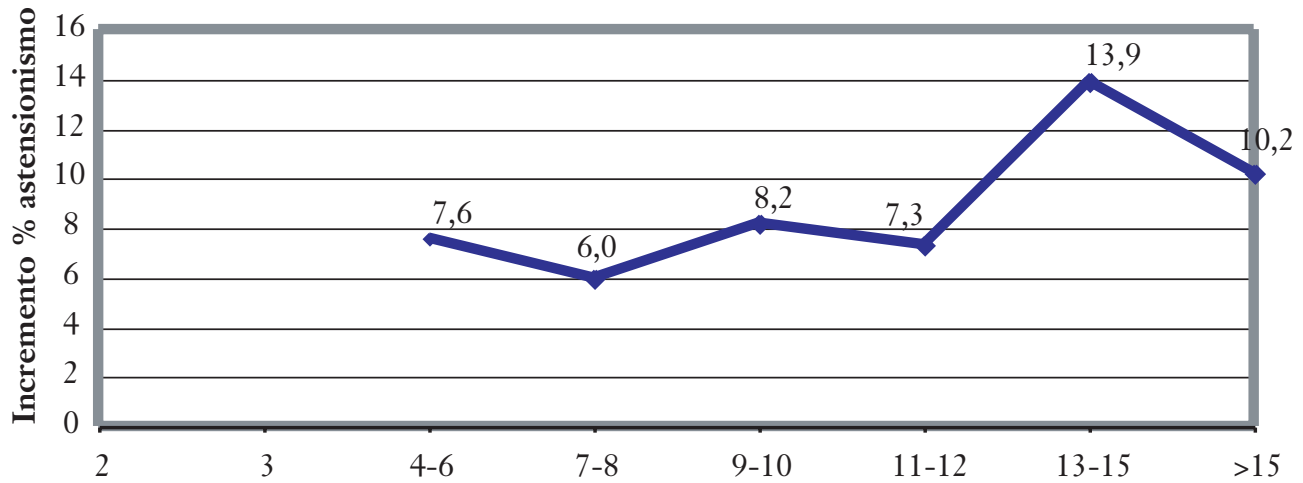

Numero liste concorrenti

FIG. 7b. - Toscana. Numero dei candidati alle elezioni amministrative (2000-2004) e incremento dell'astensionismo rispetto alle elezioni politiche del 2001. Comuni con oltre 15mila abitanti.

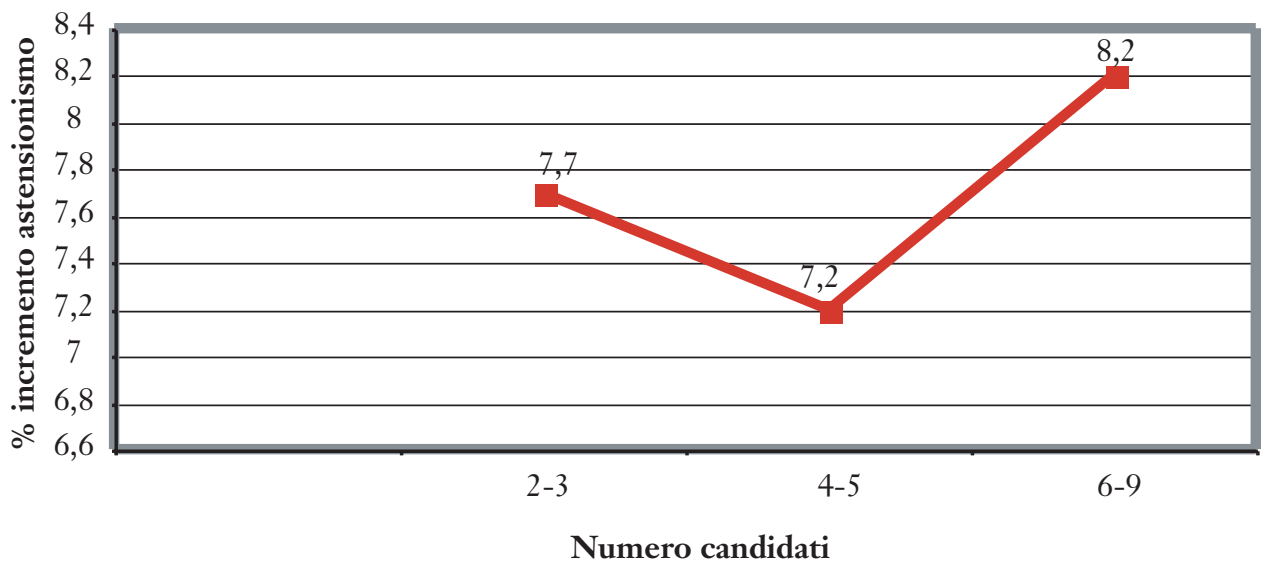

Una nuova modalità di partecipazione: il voto "personale" ai sindaci. - Come abbiamo ricordato, i processi di differenziazione nei livelli e nelle forme della partecipazione elettorale non investono solo elezioni diverse, in tempi diversi, ma si esprimono anche nell'ambito di una stessa elezione: in particolare, con le nuove regole delle elezioni amministrative, la possibilità di voto personalizzato e di voto diviso e, 
ovviamente, quella di un voto univoco $^{16}$, consente al singolo elettore di modulare come meglio crede la propria risposta alla proposta politica che si trova di fronte. Così, appare plausibile l'ipotesi che, nel complesso, questa possibile articolazione delle modalità di voto possa rappresentare un freno ad un'ulteriore incremento dell'astensionismo, offrendo agli elettori una serie di opzioni differenziate: possono votare solo per un candidato sindaco quegli elettori che non apprezzano l'offerta partitica (e che magari, solo in presenza di questa, si sarebbero astenuti), o possono anche "dividere" il voto quanti si trovano ad apprezzare una candidatura, ma non quella dei partiti che la sostengono; mentre possono votare per un partito quanti invece assegnano un ruolo privilegiato a questo canale di rappresentanza politica, limitandosi ad "accettare" la candidatura al sindaco collegata alla lista prescelta. Il voto ad una lista, a sua volta, può essere fortemente trainato dal voto di preferenza per l'elezione dei consiglieri comunali, come vedremo nel prossimo paragrafo.

Il fenomeno del voto personalizzato è stato molto discusso, negli anni scorsi, soprattutto quando è emerso nel dibattito politico nazionale il tema del «partito dei sindaci»: possiamo dire oggi, dopo tre tornate elettorali, che le dimensioni del fenomeno, (pure rilevanti, nella fase di prima applicazione della legge, specie in alcune grandi città) erano state sopravvalutate, e non risultano confermate, se misurate sull'intero universo dei comuni al voto. Quanti sono gli elettori che votano solo per i candidati sindaci? E, nel corso degli anni, crescono o diminuiscono? I dati relativi alla Toscana mostrano, nel complesso, una crescita del fenomeno, anche se l'ultimo ciclo elettorale sembra segnare un rallentamento del fenomeno (FIG. 8): nei 51 comuni considerati, gli elettori che hanno votato solo per i candidati sindaco erano il 5,6\% negli anni 1993-1995, sono saliti al 6,2\% nelle elezioni svoltesi tra il 1997 e il 1999, per poi assestarsi al 6,5\% nelle elezioni del 2000-2004. Da notare come la più alta percentuale (7,2\%) si sia sempre registrata nei comuni non capoluogo di media dimensione, quelli con un consiglio Comunale composto da 30 seggi, ossia con un numero di abitanti compreso tra i 30 e i 100 mila abitanti ${ }^{17}$.

16 Definiamo come «voto personalizzato» il voto espresso solo al candidato sindaco, mentre «voto diviso» è il voto espresso per un candidato e, nel contempo, ad una lista che sostiene un altro candidato. Il voto «univoco» è invece un voto coerente, per un candidato e per una delle liste che lo sostengono: materialmente, tuttavia, l'espressione di un voto univoco può avvenire in due modi, o con un doppio voto, o con un voto alla sola lista, che si trasferisce automaticamente al candidato collegato.

${ }^{17}$ In Toscana, peraltro, gli 11 comuni compresi in questa classe, hanno mediamente quasi 44 mila abitanti, con il più popoloso, Carrara, che conta 67 mila abitanti. 
La TAB. 5 permette anche di valutare i possibili scostamenti tra il dato relativo al totale degli elettori (a cui si riferisce la FIG. 8) e la media dei dati relativi ai singoli comuni appartenenti alle diverse classi demografiche: la limitata variabilità di questi due dati mostra come il fenomeno del voto personalizzato sia piuttosto omogeneo e coinvolga in misura analoga tutti i comuni.

FIG. 8. - Toscana. Voto personalizzato. Percentuale media di voti personali ai candidati sindaco sul totale degli elettori. Comuni con oltre 15 mila abitanti.

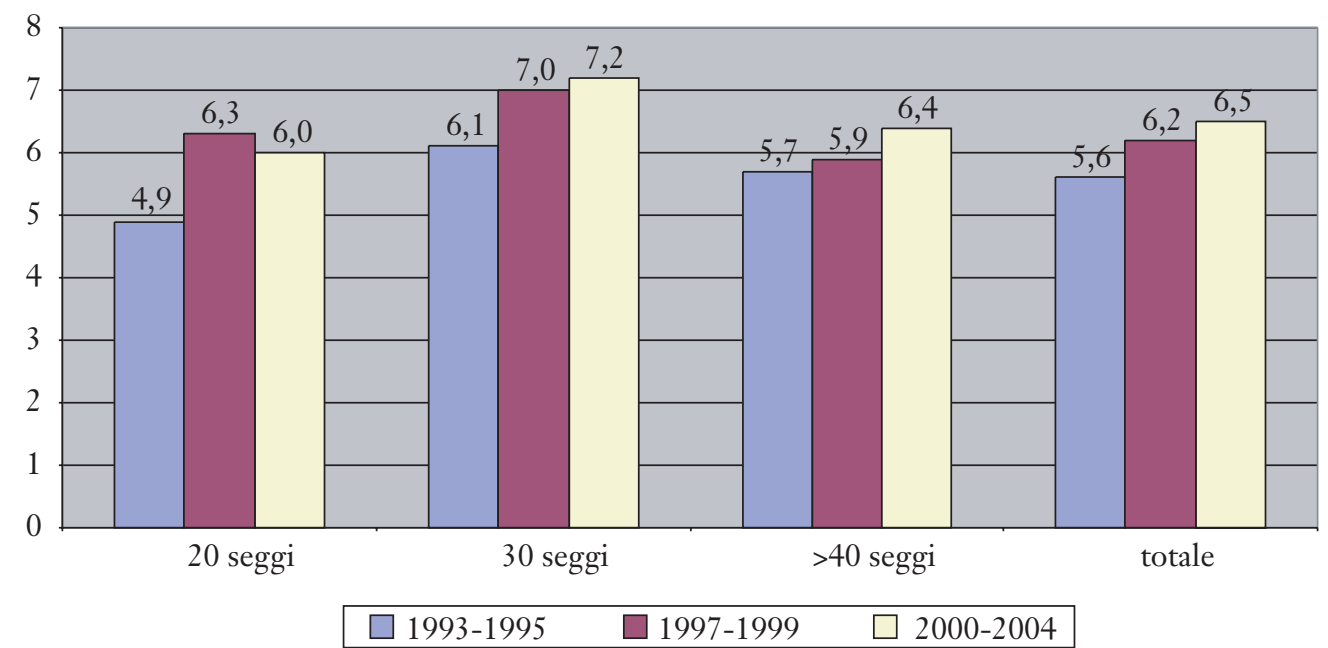

TAв. 5 - Toscana. Voto personalizzato. Percentuale di voti personali ai candidati sindaco sul totale degli elettori, per classi dimensionali dei consigli comunali. Comuni con oltre 15 mila abitanti.

\begin{tabular}{cccccccc}
\hline $\begin{array}{c}\text { Dimensione } \\
\text { Consiglio Comunale }\end{array}$ & & n. casi & $1993-1995$ & n. casi & 1997-1999 & n. casi & 2000-2004 \\
\hline 20 seggi & media & 30 & 4,8 & 30 & 6,2 & 30 & 6,1 \\
& totale & & 4,9 & & 6,3 & & 6,0 \\
\multirow{2}{*}{30 seggi } & media & 11 & 5,9 & 11 & 7,2 & 12 & 7,0 \\
& totale & & 6,1 & & 7,0 & & 7,2 \\
\multirow{2}{*}{40 seggi } & media & \multirow{2}{*}{10} & 6,0 & 10 & 6,6 & 10 & 7,1 \\
& totale & & 5,7 & & 5,9 & & 6,4 \\
\hline \multirow{2}{*}{ totale } & media & 51 & 5,3 & 51 & 6,5 & 52 & 6,5 \\
& totale & & 5,6 & & 6,2 & & 6,5 \\
\hline
\end{tabular}

È possibile anche misurare la percentuale di voti personalizzati ai candidati sindaci non più rispetto agli elettori, ma rispetto al totale dei voti validi, esclu- 
dendo dunque gli elettori assenti dalle urne e quelli che hanno espresso un voto bianco o nullo. Anche da questo versante emerge una tendenza alla stabilizzazione del fenomeno, dopo una prima fase di crescita. Infatti, se consideriamo i due primi cicli elettorali, la percentuale di voto personalizzato cresce significativamente, passando dal 7,1\% al 9,0\% (FIG. 9 e TAB. 6). Il terzo ciclo elettorale (2000-2004) segna invece un lieve regresso, soprattutto nei comuni medio-piccoli e una stasi nei comuni maggiori. Nel complesso, il dato complessivo si attesta oggi all' $8,8 \%{ }^{18}$.

Anche in questo caso, il confronto tra totale e media non rileva scostamenti significativi, a segnalare la relativa omogeneità del fenomeno.

FIG. 9. - Toscana. Voto personalizzato. Percentuale di voti personali ai candidati sindaco sul totale dei voti validi. Comuni con oltre 15 mila abitnati.

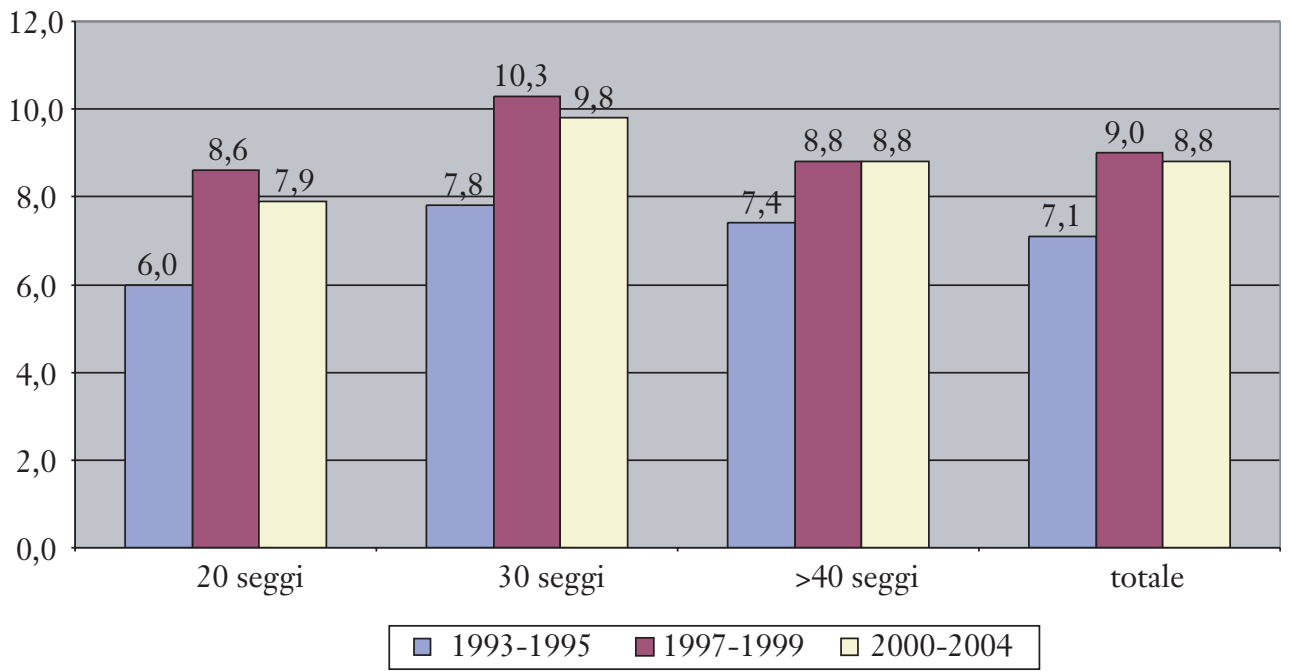

Nel complesso, dunque, il fenomeno del voto personalizzato ai candidati sindaci investe una quota di elettori pari a poco meno di un decimo degli elettori che esprimono un voto valido: un fenomeno di dimensioni relativamente circo-

18 Una diretta comparazione con i dati nazionali presentati da G. BALDINI e G. LEGNANTE, Città al voto, cit., pp. 142-144, è possibile solo per i comuni che avevano votato nel 1997-1998, e per i quali era quindi possibile un confronto con i dati del 1993-1994: Baldini e Legnante rilevavano una media di voto personalizzato del 7,2\% (sul totale degli elettori), con un incremento dell' $1,3 \%$ : rispetto ai primi due cicli elettorali dei comuni toscani qui considerati, dunque, il livello di voto personalizzato in Toscana è inferiore al dato medio nazionale $(5,6 \%$, rispetto al $7,2 \%)$ ed anche l'incremento successivo è leggermente inferiore $(+1.1 \%)$. Nel complesso, secondo i dati di Baldini e Legnante, il voto personalizzato sul totale degli elettori, negli anni 1997-1998, era del 6,4\%; negli stessi anni, sul totale dei voti validi, era dell' $8,6 \%$. 
scritte, dunque, ma che tuttavia si può rivelare molto importante dal punto di vista dell'esito stesso delle elezioni, in quanto alla diversa entità del voto personalizzato dei vari candidati è legato il "rendimento" dei candidati stessi, ovvero la loro capacità di "aggiungere" un contributo di consenso personale a quello proveniente dai partiti che li sostengono. Un fattore tanto più rilevante, ovviamente, quanto più aperta si presenta la competizione.

TAB. 6 - Toscana. Voto personalizzato. Percentuale di voti personali ai candidati sindaco sul totale dei voti validi, per classi dimensionali dei consigli comunali. Comuni con oltre 15 mila abitanti.

\begin{tabular}{ccccc}
\hline $\begin{array}{c}\text { Dimensione } \\
\text { Consiglio Comunale }\end{array}$ & 1993-1995 & 1997-1999 & 2000-2004 \\
\hline \multirow{2}{*}{20 seggi } & media & 6,0 & 8,5 & 7,9 \\
& totale & 6,0 & 8,6 & 7,9 \\
\multirow{2}{*}{30 seggi } & media & 7,7 & 10,5 & 9,7 \\
& totale & 7,8 & 10,3 & 9,8 \\
\multirow{3}{*}{$>40$ seggi } & media & 7,8 & 9,5 & 9,5 \\
& totale & 7,4 & 8,8 & 8,8 \\
\hline \multirow{2}{*}{ totale } & media & 6,7 & 9,1 & 8,6 \\
& totale & 7,1 & 9,0 & 8,8 \\
\hline
\end{tabular}

Il voto di preferenza: l'altra faccia della personalizzazione. - La possibilità di esprimere un voto di preferenza ai candidati alla carica di consigliere rappresenta l'altro, essenziale canale attraverso cui si esprime una dimensione personalizzata della partecipazione elettorale: un canale di cui non sempre si riesce ad apprezzare adeguatamente la portata, anche per la difficoltà a reperire una base di dati comparabili sufficientemente estesa, specie per le elezioni amministrative.

Le analisi disponibili confermano tuttavia come il voto di preferenza rappresenti tuttora, specie nelle regioni meridionali, il principale, se non proprio, l'unico vero canale di mediazione politica tra partiti ed elettori. Anche il voto di preferenza esprime una forma di personalizzazione della politica: l'altra e più antica forma di voto personale, si potrebbe dire, che continua a mantenere intatte, ed anzi per certi versi aggravandole, tutte quelle caratteristiche che, giustamente, ne hanno fatto uno dei più efficaci indicatori del livello di particolarismo di un sistema politico locale o regionale: tanto più deboli sono le identità collettive, tanto più elevato è l'uso del voto di preferenza; tanto più avanzato il processo di destrutturazione dei partiti come soggetti forti e unitari della rappresentanza politica, tanto più rilevante la 
diffusione del voto di preferenza come "supplenza" ad un deficit di rappresentatività dei partiti in quanto tali ${ }^{19}$.

Certo, all'interno di un sistema elettorale fondato sull'elezione diretta del sindaco, e quindi su una nuova e più moderna forma di personalizzazione della politica, le caratteristiche strutturali proprie del voto di preferenza assumono una diversa rilevanza e producono i propri effetti attraverso nuovi meccanismi e inedite interazioni all'interno del mutato contesto istituzionale: se, infatti, da una parte, l'elezione diretta del sindaco accentua la visibilità e la responsabilità politica del vertice dell'esecutivo, dall'altra parte, la frammentazione della rappresentanza consiliare, (prodotta da vari altri meccanismi, su cui ci soffermeremo ampiamente nel corso di questo lavoro) viene accentuata dalle modalità stesse di investitura dei singoli consiglieri, espressione di un (generalmente) piccolo "pacchetto" di consenso personale che cerca e deve poi trovare un proprio modo di manifestarsi e alimentarsi. Risulta dunque aggravata, anche per questa via, una contraddizione da più parti rilevata, a proposito del nuovo assetto istituzionale degli enti locali, ossia la contrapposizione (talvolta acuta e improduttiva, e comunque latente) tra il nuovo potere del sindaco e una rappresentanza consiliare frammentata e (spesso) protesa alla costante, quanto vana, ricerca di propri spazi politici o aree di influenza. Torneremo più avanti su questo tema: intanto, cerchiamo qui di valutare, per quanto possibile, le dimensioni che assume il voto di preferenza all'interno del nuovo sistema elettorale.

Per valutare compiutamente il comportamento degli elettori è opportuno distinguere tutte le possibili articolazioni delle scelte di voto: l'astensione, il voto bianco e nullo, il voto esclusivo ai candidati sindaci, il voto esclusivo ad una lista, il voto ad una lista con voto di preferenza. I dati disponibili sono relativi a 43 elezioni comunali (sulle 52 del nostro universo di riferimento) svoltesi in Toscana con il terzo ciclo elettorale (2000-2004). Sono due gli elementi di fondo che emergono da questa prima serie di dati (FIG. 10): se, da una parte, il voto in Toscana, (anche il voto amministrativo), rimane ancora, ed in larga misura, un voto mediato dalla

${ }^{19}$ Per un'analisi "storica" del voto di preferenza in Italia, si vedano P. SCARAMOZZINO, Un'analisi statistica del voto di preferenza in Italia, Milano, Giuffrè 1979; R. D’AMICO, «Voto di preferenza, movimento dell'elettorato e modelli di partito. L'andamento delle preferenze nelle elezioni politiche italiane del quindicennio 1968-1983», in Quaderni dell'Osservatorio elettorale, 18, 1987, pp. 89-147. Per un'analisi del voto di preferenza come indicatore (per quanto «ambiguo») della diffusione del «voto di scambio», si veda in particolare R. CARTOCCI, Elettori in Italia. Riflessioni sulle vicende elettorali degli anni Ottanta, Bologna, Il Mulino, 1990: Cartocci ricostruisce i termini di una lunga disputa interpretativa sui caratteri del voto di preferenza, ma ricorda anche come, sul finire degli anni Ottanta, si levassero già numerose voci, anche nella commissione Bozzi per le riforme istituzionali, tendenti a proporre l'abolizione del voto di preferenza. Dai dati sull'uso del voto di preferenza, Cartocci trae alcune considerazioni: «la distribuzione territoriale del tasso di preferenza è congruente con le conclusioni cui pervengono gli studi sulla natura e i meccanismi del clientelismo; nel dibattito in merito alla riforma del sistema elettorale, il voto di preferenza viene sistematicamente denunciato come un incentivo alla corruzione e alla degenerazione dei rapporti tra eletti ed elettori; la cronaca delle contestazioni dei risultati elettorali rafforza l'interpretazione del voto di preferenza in termini di voto di scambio» (p. 106). Cartocci presenta poi due cartogrammi, molto eloquenti, sulla diffusione del voto di preferenza in Italia per la DC e il PCI nelle elezioni politiche del 1979. 
scelta partitica, dall'altra parte, viene una conferma di quanto l'uso del voto di preferenza sia una modalità di voto fortemente legata alla specificità dei sistemi politici locali e alle tradizioni di cultura politica che in essi risultano prevalenti.

FIG. 10 - Toscana. Modalità di partecipazione al voto (2000-2004). Astensioni, voti non validi, voto esclusivo ai sindaci e alle liste, voto alle liste con preferenza (\%). Comuni con oltre 15 mila abitanti (43 casi).

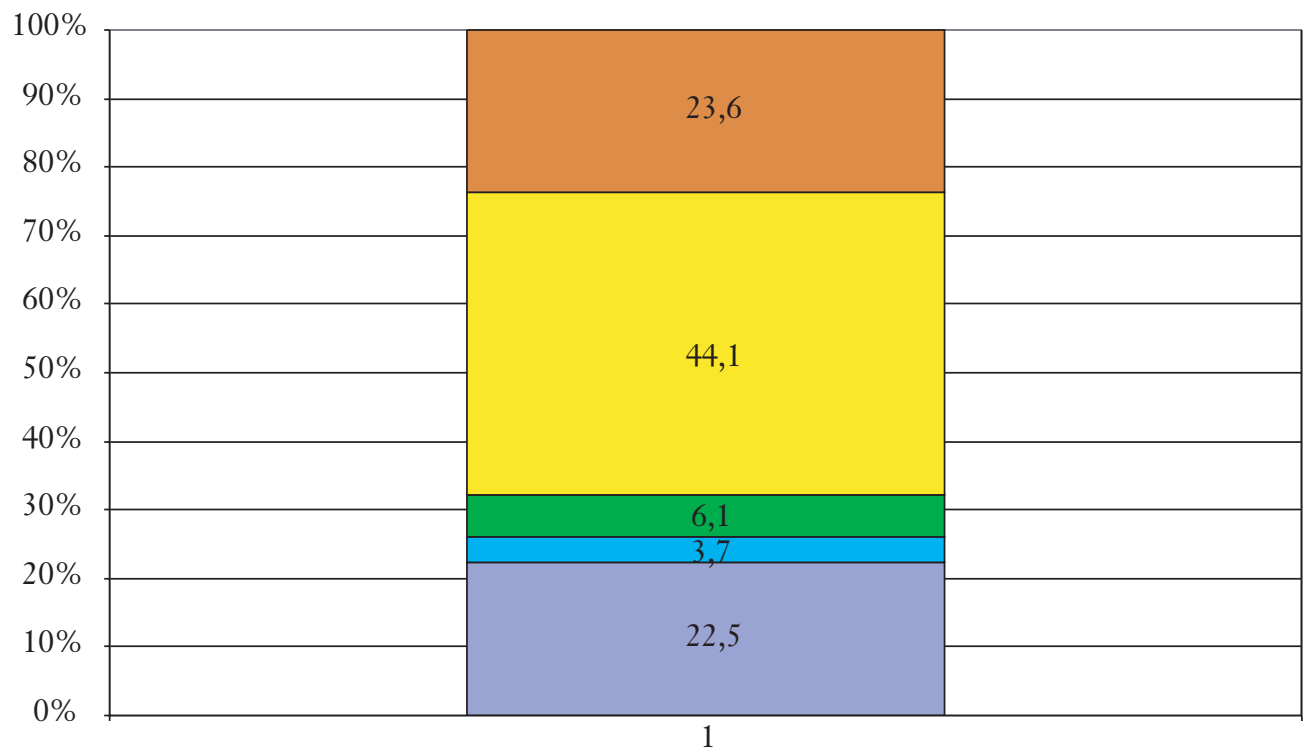

$\square$ astenuti $\square$ bianche e nulle $\quad \square$ voto solo sindaco $\quad \square$ voto solo lista $\quad \square$ voti di lista con pref

Nel complesso, su 100 elettori toscani, sono ben il 44\% quelli che esprimono un voto esclusivo alle liste di partito, a fronte del $24 \%$ che scelgono anche un candidato consigliere e del $6 \%$ che votano solo per i candidati sindaci: dati che confermano un profilo tradizionale della Toscana come regione in cui il voto di preferenza non sembra svolgere un ruolo particolarmente significativo nella mediazione tra partiti, istituzioni e società civile. L'unico possibile termine di confronto, per le elezioni amministrative svoltesi con il nuovo sistema elettorale, è costituito dalla ricerca condotta da Baldini e Legnante, dalla quale risulta, nei 48 capoluoghi per i quali sono stati disponibili i dati, una percentuale di voto esclusivo ai partiti pari al 38,3\% nelle regioni del Centro, del 34\% nelle regioni del Nord, del 13,6\% in Sicilia e di solo il 10,3\% nelle altre regioni del Sud ${ }^{20}$.

${ }^{20}$ G. BALDINI e G. LEGNANTE, Città al voto, cit., pp. 154-5. Il campione di capoluoghi utilizzato dai due autori comprende tre città toscane (Pistoia, Grosseto e Siena), e si riferisce alle elezioni del 1997 (Grosseto e Siena) e del 1998 (Pistoia). In queste città, gli elettori che hanno votato "solo" i partiti sono stati il 37,3\% (a Pistoia), il 40,1\% (a Grosseto) e il 39,3\% (a Siena). Gli elettori che hanno espresso una preferenza sono stati il $18,8 \%$ a Pistoia, il $34,1 \%$ a Grosseto e il $32,3 \%$ a Siena. Ringrazio Guido Legnante per aver gentilmente messo a disposizione i dati relativi alle tre citate città toscane. 
D'altra parte, come si accennava, anche all'interno della Toscana contano le specificità dei sistemi politici locali e delle diverse tradizioni di cultura politica (ТАB. 7): considerando l'uso del voto di preferenza nelle diverse province toscane, emerge chiaramente una linea di frattura che divide le aree in cui più marcata e profonda è stata l'influenza della "subcultura rossa" da quelle in cui altre erano e sono le culture politiche dominanti (Lucca e la Lucchesia) o comunque da quelle in cui i sistemi politici locali sono state sempre, storicamente, caratterizzati da un maggiore equilibrio nei rapporti di forza (Massa Carrara). Abbiamo così i comuni di quattro province "rosse" in cui oltre il 50\% degli elettori ha votato solo per una lista di partito (nell'ordine, Siena, Firenze, Livorno e Prato) mentre meno del 20\% hanno espresso un voto di lista con preferenza; e una provincia (Arezzo) in cui il $46 \%$ degli elettori ha votato solo le liste e il $25 \%$ ha espresso anche una preferenza; seguono poi i quattro comuni della provincia di Pisa, con una media provinciale del $38 \%$ che però risulta da dati molto divergenti del capoluogo $(31 \%)$ e degli altri tre comuni (tutti oltre il 45\%). I due comuni grossetani (il capoluogo e Follonica) vedono un rapporto più equilibrato tra le due tipologie di voto, così come nella media provinciale di Pistoia (per la quale, non essendo disponibili i dati del capoluogo e di Quarrata, incidono particolarmente i dati di Montecatini Terme, comune in cui prevale il centrodestra, e di Pescia, comune che ha visto varie alternanze nel corso del decennio). Infine, i due casi già ricordati di Lucca e Massa Carrara: qui sono decisamente più numerosi gli elettori che utilizzano il voto di preferenza, rispetto a quelli che votano solo una lista. Con una differenza significativa, tuttavia: nei comuni lucchesi (e qui incide il dato di Viareggio) è molto più elevato il voto esclusivo ai sindaci; mentre a Massa e Carrara quasi la metà degli elettori usa la preferenza (per i dati completi, comune per comune, si veda la TAB. 8).

TAB. 7 - Toscana. Modalità di partecipazione al voto (2000-2004). Astensioni, voti non validi, voto esclusivo ai sindaci e alle liste, voto alle liste con preferenza (\%). Province (43 casi).

\begin{tabular}{lccrccc}
\hline & n. casi & astenuti & $\begin{array}{c}\text { bianche } \\
\text { nulle }\end{array}$ & $\begin{array}{c}\text { voto solo } \\
\text { sindaco }\end{array}$ & $\begin{array}{c}\text { voto } \\
\text { solo lista }\end{array}$ & $\begin{array}{c}\text { voti di lista } \\
\text { con pref. }\end{array}$ \\
\hline Arezzo & 5 & 18,8 & 3,7 & 6,2 & 46,2 & 25,0 \\
Firenze & 14 & 21,5 & 3,7 & 5,1 & 51,2 & 18,4 \\
Grosseto & 2 & 13,7 & 3,5 & 12,9 & 37,3 & 32,7 \\
Livorno & 5 & 23,8 & 3,7 & 4,8 & 50,5 & 17,2 \\
Lucca & 4 & 29,8 & 3,3 & 11,2 & 24,0 & 31,7 \\
Massa Carrara & 2 & 23,8 & 4,1 & 6,3 & 18,9 & 46,8 \\
Pisa & 4 & 25,6 & 2,9 & 5,2 & 38,4 & 27,8 \\
Pistoia & 3 & 23,4 & 4,4 & 6,8 & 34,4 & 30,9 \\
Prato & 2 & 22,5 & 4,4 & 4,8 & 50,6 & 17,8 \\
Siena & 2 & 17,5 & 4,6 & 3,9 & 56,4 & 17,7 \\
\hline Toscana & 43 & 22,5 & 3,7 & 6,1 & 44,1 & 23,6 \\
\hline
\end{tabular}


TAB. 8 - Toscana. Modalità di partecipazione al voto (2000-2004). Astensioni, voti non validi, voto esclusivo ai sindaci e alle liste, voto alle liste con preferenza (\%). Comuni con oltre 15 mila abitanti (43 casi).

\begin{tabular}{|c|c|c|c|c|c|}
\hline & astenuti & bianche e nulle & $\begin{array}{c}\text { voto solo } \\
\text { sindaco }\end{array}$ & $\begin{array}{c}\text { voto } \\
\text { solo lista }\end{array}$ & $\begin{array}{l}\text { voti di lista } \\
\text { con pref. }\end{array}$ \\
\hline Arezzo & 19,7 & 2,9 & 5,0 & 46,2 & 26,2 \\
\hline Bagno a Ripoli & 18,3 & 2,8 & 5,5 & 58,1 & 15,2 \\
\hline Borgo San Lorenzo & 18,2 & 5,3 & 5,6 & 47,4 & 23,5 \\
\hline Calenzano & 17,4 & 3,0 & 4,2 & 56,0 & 19,4 \\
\hline Camaiore & 25,8 & 3,3 & 15,8 & 23,5 & 31,6 \\
\hline Campi Bisenzio & 21,2 & 3,8 & 5,4 & 46,6 & 22,9 \\
\hline Capannori & 26,5 & 4,5 & 9,3 & 23,8 & 36,0 \\
\hline Carrara & 26,3 & 3,5 & 8,5 & 21,3 & 40,4 \\
\hline Castelfiorentino & 17,1 & 4,0 & 3,9 & 60,4 & 14,6 \\
\hline Cecina & 21,3 & 3,8 & 6,3 & 48,8 & 19,8 \\
\hline Certaldo & 16,1 & 3,2 & 4,0 & 63,4 & 13,3 \\
\hline Colle di Val D'Elsa & 17,4 & 4,2 & 3,7 & 54,8 & 19,9 \\
\hline Collesalvetti & 19,2 & 3,8 & 3,9 & 49,6 & 23,5 \\
\hline Cortona & 22,5 & 4,4 & 2,9 & 40,4 & 29,8 \\
\hline Empoli & 18,2 & 3,8 & 5,9 & 55,4 & 16,8 \\
\hline Firenze & 24,0 & 3,7 & 5,6 & 49,5 & 17,2 \\
\hline Follonica & 19,9 & 3,5 & 7,6 & 38,3 & 30,7 \\
\hline Fucecchio & 21,8 & 4,1 & 4,0 & 53,8 & 16,3 \\
\hline Grosseto & 11,8 & 3,5 & 14,4 & 37,0 & 33,3 \\
\hline Lastra a Signa & 19,1 & 4,2 & 6,0 & 51,9 & 18,8 \\
\hline Livorno & 25,7 & 3,3 & 5,0 & 51,9 & 14,2 \\
\hline Massa & 21,4 & 4,6 & 4,3 & 16,5 & 53,1 \\
\hline Massarosa & 20,0 & 3,5 & 6,9 & 23,6 & 46,1 \\
\hline Monsummano Terme & 21,7 & 5,3 & 5,8 & 39,2 & 27,9 \\
\hline Montecatini-Terme & 24,1 & 2,9 & 8,6 & 29,2 & 35,3 \\
\hline Montemurlo & 19,8 & 4,6 & 4,3 & 44,3 & 27,0 \\
\hline Montevarchi & 11,7 & 4,1 & 10,1 & 57,0 & 17,2 \\
\hline Pescia & 24,4 & 5,1 & 6,1 & 35,0 & 29,4 \\
\hline Piombino & 20,6 & 4,8 & 3,4 & 49,4 & 21,8 \\
\hline Pisa & 30,9 & 2,0 & 6,5 & 31,0 & 29,6 \\
\hline Poggibonsi & 17,5 & 4,9 & 4,0 & 57,5 & 16,1 \\
\hline Pontassieve & 16,3 & 4,0 & 3,6 & 35,8 & 40,4 \\
\hline Pontedera & 20,5 & 4,7 & 4,7 & 45,2 & 24,9 \\
\hline Prato & 22,7 & 4,4 & 4,8 & 51,2 & 16,8 \\
\hline Rosignano Marittimo & 22,3 & 4,6 & 4,1 & 46,3 & 22,6 \\
\hline San Giovanni Valdarno & 19,1 & 5,4 & 5,3 & 49,7 & 20,5 \\
\hline San Giuliano Terme & 20,8 & 3,5 & 3,6 & 46,1 & 25,9 \\
\hline San Miniato & 17,8 & 3,9 & 3,3 & 48,6 & 26,5 \\
\hline Sansepolcro & 18,2 & 5,2 & 12,8 & 36,4 & 27,3 \\
\hline Scandicci & 19,3 & 3,5 & 3,4 & 55,1 & 18,7 \\
\hline Sesto Fiorentino & 18,5 & 3,3 & 4,0 & 57,8 & 16,4 \\
\hline Viareggio & 37,3 & 2,3 & 11,9 & 24,5 & 23,9 \\
\hline Signa & 19,4 & 6,0 & 5,0 & 42,8 & 26,8 \\
\hline TOTALE & 22,5 & 3,7 & 6,1 & 44,1 & 23,6 \\
\hline
\end{tabular}


Ma, l'uso del voto di preferenza, è cresciuto, con il passare degli anni? Purtroppo i dati disponibili non consentono ancora un confronto a largo raggio: sono solo 10 i comuni toscani per i quali, nel momento in cui scriviamo, è possibile una comparazione relativa agli ultimi due cicli elettorali. Pur con questi limiti, ciò che emerge è significativo (ТАB. 9): nel complesso, il tasso di preferenza (la percentuale di preferenze espresse sul totale dei voti alle liste) sale, in questi comuni, dal $32,6 \%$ al 37,3\%, confermandosi però le notevoli diversità nel comportamento elettorale delle diverse aree della regione. Mentre a Massa ben tre elettori su quattro usano il voto di preferenza, a Firenze sono solo uno su quattro; a Massa, pur partendo da un tasso del $70 \%$, si sale ancora di altri sei punti percentuali; a Firenze, si passa solo dal 23,1 al 25,8\% (eppure, ricordiamo, vi era stata una sovrabbondante offerta di liste...: ben 27). A Pisa, Grosseto e Viareggio poco meno della metà dei voti alle liste contengono anche un voto di preferenza; mentre il dato più basso, in assoluto, tra quelli disponibili, è quello del comune di Empoli, con un tasso del 23,2\%.

TAв. 9 - Toscana. Voti di lista e voti di preferenza. Confronto tra i cicli elettorali 19971999 e 2000-2004. Comuni con oltre 15 mila abitanti (10 casi).

\begin{tabular}{|c|c|c|c|c|c|c|}
\hline & \multicolumn{3}{|c|}{ 1997-1999 } & \multicolumn{3}{|c|}{$2000-2004$} \\
\hline & $\begin{array}{l}\text { totale voti } \\
\text { di lista }\end{array}$ & $\begin{array}{l}\text { totale } \\
\text { preferenze } \\
\text { espresse }\end{array}$ & $\begin{array}{c}\text { tasso di uso } \\
\text { preferenze } \\
\text { (\% su voti } \\
\text { validi alle liste) }\end{array}$ & $\begin{array}{l}\text { totale voti } \\
\text { di lista }\end{array}$ & $\begin{array}{l}\text { totale } \\
\text { preferenze } \\
\text { espresse }\end{array}$ & $\begin{array}{c}\text { tasso di uso } \\
\text { preferenze } \\
\text { (\% su voti } \\
\text { validi alle liste) }\end{array}$ \\
\hline Camaiore & 14.002 & 2.852 & 20,4 & 14.342 & 8.220 & 57,3 \\
\hline Campi Bisenzio & 18.716 & 5.322 & 28,4 & 21.624 & 7.134 & 33,0 \\
\hline Empoli & 25.333 & 4.901 & 19,3 & 26.612 & 6.182 & 23,2 \\
\hline Firenze & 194.178 & 44.944 & 23,1 & 204.797 & 52.917 & 25,8 \\
\hline Grosseto & 45.957 & 21.156 & 46,0 & 43.762 & 20.747 & 47,4 \\
\hline Massa & 38.998 & 27.341 & 70,1 & 41.239 & 31.455 & 76,3 \\
\hline Pisa & 48.488 & 20.447 & 42,2 & 47.377 & 23.139 & 48,8 \\
\hline $\begin{array}{l}\text { San Giuliano } \\
\text { Terme }\end{array}$ & 15.926 & 4.353 & 27,3 & 18.502 & 6.655 & 36,0 \\
\hline Scandicci & 27.405 & 5.376 & 19,6 & 30.730 & 7.786 & 25,3 \\
\hline Viareggio & 28.766 & 12.655 & 44,0 & 26.179 & 12.921 & 49,4 \\
\hline TOTALE (10 casi) & 457.769 & 149.347 & 32,6 & 475.164 & 177.156 & 37,3 \\
\hline
\end{tabular}

Infine, se vi è una specificità territoriale, è evidente come vi sia anche una stretta connessione tra l'uso del voto di preferenza e la forza elettorale relativa dei diversi partiti: i partiti che sono eredi della tradizione politica democristiana e socialista, in particolare, continuano ad essere caratterizzati da un elettorato sensibile al voto di preferenza come canale fondamentale di mediazione e di rapporto con il proprio partito. Sia all'interno del centrodestra (UDC) che all'interno del centrosinistra (Margherita e SDI), sono questi partiti a registrare il più elevato 
tasso di preferenze (TAB. 10). Partiti con un più marcato profilo identitario (AN e Lega, nel centrodestra; DS, Verdi, PRC e PDCI, nel centrosinistra) o con una più marcata presenza di elettorato di opinione, come la stessa Forza Italia, non superano un tasso di preferenza pari ad un terzo del proprio consenso complessivo e, per alcuni di essi, specie quelli che si richiamano alla tradizione comunista, sono anche inferiori al $20 \%$.

TAв. 10 - Toscana. Tasso di uso del voto di preferenza per partito o tipo di lista. Comuni con oltre 15 mila abitanti (43 casi).

\begin{tabular}{lrc}
\hline \multicolumn{1}{c}{ Partito } & n. casi & $\begin{array}{c}\text { Tasso di uso del voto } \\
\text { di preferenza }\end{array}$ \\
\hline Alleanza Nazionale & 32 & 33,2 \\
UDC & 22 & 47,5 \\
Forza Italia & 32 & 28,1 \\
An-Udc & 3 & 40,9 \\
Lega Nord Toscana & 13 & 24,6 \\
Unitarie CD & 9 & 30,3 \\
Locali CD & 12 & 42,7 \\
\hline Margherita & 38 & 55,9 \\
DS & 37 & 33,3 \\
PdCI & 40 & 18,7 \\
PRC & 43 & 19,2 \\
SDI & 27 & 66,0 \\
Verdi & 26 & 23,7 \\
IdV & 16 & 16,4 \\
Unitarie CS & 5 & 33,8 \\
Locali CS & 23 & 47,3 \\
\hline TOTALE & 480 & 34,9 \\
\hline
\end{tabular}

Riassumendo, si può dire che il voto di preferenza, anche nel mutato contesto istituzionale creato dalle nuove regole elettorali, costituisce un importante veicolo di mediazione tra partiti ed elettori, riproducendo tutte le classiche "distorsioni" che il voto di preferenza ha comportato: come ora vedremo meglio, le nuove regole incentivano la frammentazione dell'offerta elettorale, rendendo "conveniente" la proliferazione delle liste a sostegno dei candidati sindaco, e quindi, per questa via, incrementando anche il numero dei candidati consiglieri a caccia di preferenze. Questo meccanismo - che non spinge certo verso una maggiore coesione, forza e compattezza dei sistemi politici locali, non facilita una più elevata qualità delle politiche e non favorisce certo un ruolo autorevole del momento della rappresentanza, rispetto alla forza dell'esecutivo -, ha certamente contribuito, negli ultimi anni, (per quanto possiamo desumere dai dati disponibili) ad una relativa crescita del "tasso di uso" del voto di preferenza; tuttavia, dai dati toscani, possiamo anche dedurre come l'"abitudine" ad esprimere tale voto sia in 
larga misura tuttora legata ad alcune variabili di "lungo periodo": la specificità dei sistemi politici locali, le tradizioni di cultura politica dominanti, l'eredità di alcuni partiti della Prima Repubblica.

\section{Il mutamento nell'offerta elettorale: forme e luoghi della rappresentanza}

Sindaci e consigli comunali: un nuovo equilibrio politico ed istituzionale. - L'introduzione dell'elezione diretta del sindaco non costituisce l'unica innovazione istituzionale prodotta dalla legge 81: altrettanto importanti, per quanto spesso sottovalutate, sono state le nuove regole previste per l'elezione dei consigli comunali. In passato, com'è noto, l'assemblea rappresentativa comunale era l'arena decisiva della politica locale: era dai rapporti di forza che si creavano in consiglio che dipendeva l'elezione del sindaco e della giunta, (ma erano i partiti a determinare le sorti dei governi comunali, e non si potesse certo dire che i consigli comunali, in quanto tali, fossero un luogo in cui si assumessero le decisioni). Da qui, anche, i principali effetti di degenerazione che il precedente assetto istituzionale portava con sé, a cominciare dalla cronica instabilità delle giunte, legate al mutevole equilibrio tra i partiti, alla difficile mediazione tra gli interessi che si esprimevano nei consigli e alla diffusa "deresponsabilizzazione" che tutto ciò comportava, nella conduzione della politica e delle politiche locali. Il principio dell'elezione diretta e del premio di maggioranza assegnato al sindaco eletto, hanno cambiato radicalmente il baricentro della politica locale: il momento della rappresentanza viene subordinato a quello della decisione, il momento della delega ai partiti cede il passo a quello dell'investitura diretta degli esecutivi. La composizione del consiglio viene in primo luogo determinata dall'esito di un'altra competizione, quella tra i candidati alla carica di sindaco. L'intero meccanismo elettorale è dunque orientato primariamente alla definizione degli assetti del governo: da qui una profonda trasformazione del ruolo dei consigli comunali, da molti protagonisti vissuta come un sostanziale ridimensionamento della loro funzione e della loro "centralità" nella vita politica locale.

Non possiamo qui soffermarci sui termini di questo dibattito, che ha visto e vede impegnati le stesse assemblee elettive $\operatorname{locali}^{21}$ : in questo capitolo, piuttosto, cercheremo di vedere come e quanto è cambiata la rappresentanza nei consigli

${ }^{21}$ Si veda, ad esempio, il volume Democrazia e governo locale. Il ruolo delle assemblee elettive, a cura di A.Brasca e M. Morisi, Bologna, Il Mulino 2003, che raccoglie le relazioni e gli interventi di un convegno promosso dal consiglio comunale di Firenze, dal consiglio provinciale di Firenze e dal consiglio delle Autonomie Locali della Toscana, tenutosi a Palazzo Vecchio il 18 gennaio 2002. Sulle diffuse «lamentazioni» circa la crisi delle assemblee elettive, si veda in particolare il saggio di C. FUSARO, L'elezione diretta del capo dell'esecutivo e il ruolo delle assemblee rappresentative, (ivi, pp.147-183) e le conclusioni cui giunge: «Un'assemblea rappresentativa non è debole perché di fronte ha un esecutivo che non dipende da essa per la sua formazione. È forte ed ha un ruolo perché è in grado di assolvere alle funzioni sue proprie.... Nulla sarebbe invece più dannoso e soprattutto vano che inseguire il recupero di ciò che le assemblee hanno ampiamente dimostrato di non saper e poter fare» (p. 183). 
comunali della Toscana, a seguito delle nuove regole elettorali, e come questo mutamento si combini con la nuova figura dei sindaci.

Rappresentanza, frammentazione, stabilità. - Il tema della frammentazione della rappresentanza è cruciale da molti punti di vista, per tracciare un primo bilancio degli effetti prodotti dalla nuova legge elettorale sui sistemi politici locali: infatti, una delle ragioni che più spinsero alla riforma del 1993 fu la considerazione degli effetti di instabilità che la logica della rappresentanza proporzionale, unitamente alle dimensioni delle assemblee elettive, produceva sui governi locali. Assemblee ampie e frammentate erano il luogo, ed erano nello stesso tempo la cassa di risonanza, di estenuanti contrattazioni tra i partiti, sugli incarichi esecutivi e sulle posizioni di sottogoverno, che esponevano sindaci e giunte ad una costante minaccia di crisi. Ma soprattutto, era la qualità stessa delle policies locali a soffrirne: l'orizzonte temporale delle strategie e delle politiche si restringeva, sindaci e giunte erano indotti a privilegiare logiche di breve periodo e politiche particolaristiche, che potessero assicurare una riproduzione del consenso in chiave di scambio politico. Questa qui descritta, naturalmente, era una condizione generale degli enti locali del nostro paese, un'immagine diffusa e una "diagnosi" a cui intenderanno appunto porre un argine le proposte di modifica e di intervento sull'assetto istituzionale e sulle regole elettorali; ma, evidentemente, numerose erano le varianti regionali e locali: in particolare, per una regione come la Toscana, contavano anche altri fattori, che riducevano notevolmente la cronica instabilità che altrove in Italia si poteva registrare. E tra questi fattori, evidentemente, pesavano anche i peculiari rapporti di forza elettorali tra $\mathrm{i}$ partiti, il grado e la qualità stessa del consenso su cui si basavano gli equilibri di potere nei governi locali. Per la Toscana, a lungo, nel dopoguerra (e poi fino agli anni Ottanta, sia pure in misura decrescente), pesava anche un consenso di tipo ideologico, una "riserva" di consenso legata alla peculiare sub-cultura politica territoriale della regione, che garantiva continuità e stabilità alla gran parte degli enti locali e permetteva anche, laddove ce ne fossero le capacità politiche, di perseguire strategie e policies di medio-lungo periodo, volte alla produzione di beni e servizi collettivi, in una chiave universalistica e non particolaristica.

Alla diagnosi sullo stato critico dell'assetto istituzionale dei governi locali del nostro paese, il legislatore del 1993 rispose configurando nuove regole elettorali e forme istituzionali che garantissero, innanzi tutto, stabilità agli esecutivi e alla figura del sindaco, ma anche ne accentuassero la piena "responsabilizzazione" di fronte agli elettori: un sindaco eletto e legittimato direttamente dai cittadini, garantito da una maggioranza ampia in consiglio, in grado di "render conto" pienamente del proprio operato. E stabilità e responsabilità politica erano viste come la premessa perché si producessero anche buone politiche. Le nuove regole elettorali, dunque, dovevano agire in questo senso: e l'adozione di un sistema a doppio turno, con premio di maggioranza, d'altra parte, doveva tendere ad una strutturazione bipolare della competizione. Tuttavia, come spesso accade, obiettivi e preferenze degli attori in gioco condizionavano inevitabilmente l'esito dei processi di riforma elettorale e istituzionale: e così, accanto alla logica bipolare, il nuovo sistema elettorale accettava, e per 
così dire introiettava, il carattere frammentato del sistema dei partiti. Le nuove regole riflettevano, ma nello stesso tempo in tal modo contribuiranno a riprodurla e ad alimentarla, la peculiare natura del sistema politico italiano, nella travagliata transizione degli anni Novanta, ossia il suo essere caratterizzato da un «bipolarismo frammentato» ${ }^{22}$.

Sarebbe tuttavia un errore sostenere che il problema di una limitazione del livello di frammentazione della rappresentanza non fosse presente negli intenti riformatori della legge 81: si tratta di comprendere come esso sia stato affrontato e quali effetti siano poi stati concretamente prodotti dalle innovazioni introdotte.

Una prima novità riguardava la composizione stessa dei Consigli comunali, con una riduzione significativa del numero dei componenti le assemblee: una misura che, indubbiamente, era rivolta a ridurre la pletoricità di molti consessi cittadini, con le conseguenze politiche e istituzionali che da ciò spesso derivavano. A ciò si legava un cambiamento nella soglia demografica di applicazione delle diverse formule elettorali. Il Quadro 1 sintetizza alcuni aspetti della situazione pre-riforma:

QUADRO 1. - Formula elettorale e ampiezza delle assemblee prima del 1993.

\begin{tabular}{lll}
\hline & \multicolumn{2}{c}{ Soglia demografica di applicazione } \\
\cline { 2 - 3 } & $<5.000$ abitanti & $>5.000$ abitanti \\
\hline Formula elettorale & Maggioritaria Plurinominale & Proporzionale (divisore D’Hondt) \\
\hline Dimensioni assemblea & 15 seggi - Comuni $<3.000 \mathrm{ab}$. & 20 seggi - Comuni $<5.000 \mathrm{ab}$. \\
& 20 seggi - Comuni $>10.000 \mathrm{ab}$. & 30 seggi - Comuni $<30.000 \mathrm{ab}$. \\
& & 40 seggi - Comuni $<100.000 \mathrm{ab}$. e capoluoghi \\
& 50 seggi - Comuni $<250.000 \mathrm{ab}$. \\
& 60 seggi - Comuni $<500.000 \mathrm{ab}$. \\
& 80 seggi - Comuni $>500.000 \mathrm{ab}$. \\
\hline
\end{tabular}

Dopo il 1993 cambia molto, sia perché muta il tipo di sistema maggioritario adottato nei comuni minori e si innalza la soglia demografica che distingue i diversi sistemi elettorali, sia perché si procede ad una notevole riduzione dell'ampiezza dei consigli comunali (Quadro 2):

QUADRO 2. - Formula elettorale e ampiezza delle assemblee dopo il 1993.

\begin{tabular}{lll}
\hline & \multicolumn{2}{c}{ Soglia demografica di applicazione } \\
\cline { 2 - 3 } Formula elettorale & Mista $(2 / 3$ maggioritaria; & $>15.000$ abitanti \\
& $1 / 3$ proporzionale - div. D'Hondt) & Mista (maggioritaria $60 \% ;$ \\
Dimensioni assemblea & 12 seggi - Comuni $<3.000 \mathrm{ab}$. & 20 seggi Comuni $>15.000 \mathrm{ab}$. \\
& 16 seggi - Comuni $<10.000 \mathrm{ab}$. & 30 seggi Comuni $>30.000 \mathrm{ab}$. \\
& 20 seggi - Comuni $10.000-15.000 \mathrm{ab}$. & 40 seggi Comuni $>100.000 \mathrm{ab}$. e capoluoghi \\
& & 46 seggi Comuni $>250.000 \mathrm{ab}$. \\
& 50 seggi Comuni $>500.000 \mathrm{ab}$. \\
& 60 seggi Comuni $>1.000 .000 \mathrm{ab}$. \\
\hline
\end{tabular}

22 Così accadrà anche, per altro verso, con la riforma della legge elettorale regionale (la n. 43 del 1995), su cui si veda di R. D'ALIMONTE e A.CHIARAMONTE (a cura di), Il maggioritario regionale, Bologna, Il Mulino, 2000. 
Se confrontiamo i dati relativi ai 51 comuni toscani con oltre 15 mila abitanti, otteniamo questo quadro complessivo di riduzione del numero dei consiglieri (Quadro 3):

QUADRO 3. - N. dei consiglieri nei comuni toscani con oltre 15mila abitanti, prima e dopo la riforma del 1993.

\begin{tabular}{|c|c|c|c|c|c|}
\hline Soglia demografica & $\begin{array}{l}\text { Numero } \\
\text { comuni }\end{array}$ & $\begin{array}{c}\text { Ampiezza < } 93 \\
\text { (n. seggi) }\end{array}$ & $\begin{array}{c}\text { Ampiezza > } 93 \\
\text { (n. seggi) }\end{array}$ & $\begin{array}{c}\text { Totale seggi } \\
<93\end{array}$ & $\begin{array}{c}\text { Totale seggi } \\
>93\end{array}$ \\
\hline $250.000-500.000$ & 1 & 60 & 46 & 60 & 46 \\
\hline $100.000-250.000$ & 3 & 50 & 40 & 150 & 120 \\
\hline 30.000-100.000 e capoluoghi & 6 & 40 & 40 & 240 & 240 \\
\hline 30.000-100.00 non capoluoghi & 11 & 40 & 30 & 440 & 330 \\
\hline $15.000-30.000$ & 30 & 30 & 20 & 900 & 600 \\
\hline Totale & 51 & & & 1.790 & 1.336 \\
\hline
\end{tabular}

Come si vede, una drastica riduzione, che risparmia soltanto 6 comuni capoluogo (Arezzo, Pistoia, Lucca, Grosseto, Massa e Siena), che conservano un'ampiezza di 40 seggi: nel complesso, un quarto di consiglieri in meno.

Alle novità introdotte nella legge del 1993, vanno poi aggiunte le importanti modifiche apportate alla vigilia del turno elettorale del 1999 (legge n. 120), che intervengono su alcuni importanti aspetti del precedente testo legislativo ${ }^{23}$ : in particolare, per quel che qui più ci interessa, l'introduzione di una soglia del $3 \%$ per l'accesso alla ripartizione dei seggi in consiglio, ma solo per le liste "isolate", ossia liste che non siano collegate a coalizioni che abbiano superato la stessa soglia del 3\%. Una misura anti-frammentazione, dunque, contraddetta tuttavia, nella stessa legge, da una notevole riduzione del numero di firme necessarie per la presentazione di una lista ${ }^{24}$.

${ }^{23}$ La legge 120/99 introduceva, tra l'altro, l'accorpamento delle elezioni in un'unica tornata primaverile e modificava le regole sulla durata dei mandati amministrativi (portandola a 5 anni), chiarendo anche le regole sul numero dei mandati (specificando che «è consentito un terzo mandato consecutivo se uno dei due mandati precedenti ha avuto la durata inferiore a due anni, sei mesi e un giorno, per causa diversa dalle dimissioni volontarie»). Inoltre, veniva modificata la normativa sul premio di maggioranza, abbassando la soglia di voti (dal $50 \%$ al $40 \%$ ) delle liste collegate al sindaco eletto al primo turno, necessaria per ottenere il premio: in tal modo si evitava la possibilità (che in effetti si era verificata, in molti comuni) che un sindaco eletto al primo turno si rivelasse "troppo popolare" rispetto alle liste che lo sostenevano, con l'effetto paradossale di privarlo di una maggioranza in consiglio. Restava invariata la norma che prevede la mancata assegnazione del premio nel caso in cui una lista o coalizione avversaria superasse la soglia del $50 \%$. Per un dettagliato commento all'intero testo legislativo e alle sue successive modifiche, si veda S. VASSALLO, Sistema elettorale, cit., pp. 717-775.

${ }^{24} \mathrm{Si}$ tratta di un numero di firme obiettivamente modesto, che si può presumere incoraggi notevolmente l'intrapresa di un'"avventura" elettorale: basti ricordare ad esempio che, per una città tra 100 e 500 mila abitanti occorrono appena da 350 a 700 firme, e che per una cittadina tra i 20 e i 40 mila abitanti le firme richieste vanno soltanto da un minimo di 175 ad un massimo di 350 elettori. 
Il primo quesito a cui rispondere, a questo punto, è il seguente: questa riduzione della posta in gioco, ossia la minore disponibilità di seggi in palio, ha scoraggiato la presentazione di liste concorrenti? In altri termini, ha prodotto una qualche limitazione del grado di frammentazione dal versante dell'offerta di rappresentanza? Il secondo quesito, a cui risponderemo successivamente, riguarda invece il grado di frammentazione effettivo che è scaturito dal voto degli elettori, ossia se ed in che misura la risposta degli elettori abbia ridotto il livello di frammentazione della rappresentanza proposto dal lato dell'offerta elettorale.

Liste concorrenti e liste rappresentate: le misure della frammentazione. - Occorre dunque fare un passo indietro e ricostruire la situazione nelle due ultime elezioni amministrative svoltesi con le vecchie regole, negli anni 1983-1985 e 1988-1990 (FIG. 11; per i dati completi, si veda la TAB. 11): nel complesso, nel primo di questi due cicli elettorali, avevamo una media di 7,6 liste concorrenti, che cresce fino all'8,8 nel secondo ciclo. In entrambe le elezioni, poi, vi era un dato lineare e coerente, che vedeva la crescita di tale media al crescere della dimensione demografica dei comuni e dell'ampiezza delle assemblee: così, Firenze, unico consiglio con 60 membri, giungeva nel 1990 alla cifra di 16 liste presentate: le altre maggiori città toscane (Prato, Livorno e Pisa) ne avevano in media 10,3; i 17 comuni con un consiglio di 40 seggi, si fermavano a 9,9, mentre i comuni più piccoli ne registravano mediamente 7,8 .

FIG. 11 - Toscana. Media delle liste presentate, per ciclo elettorale e dimensione del consiglio comunale. Comuni con oltre 15mila abitanti (1983-1985 e 1988-1990).

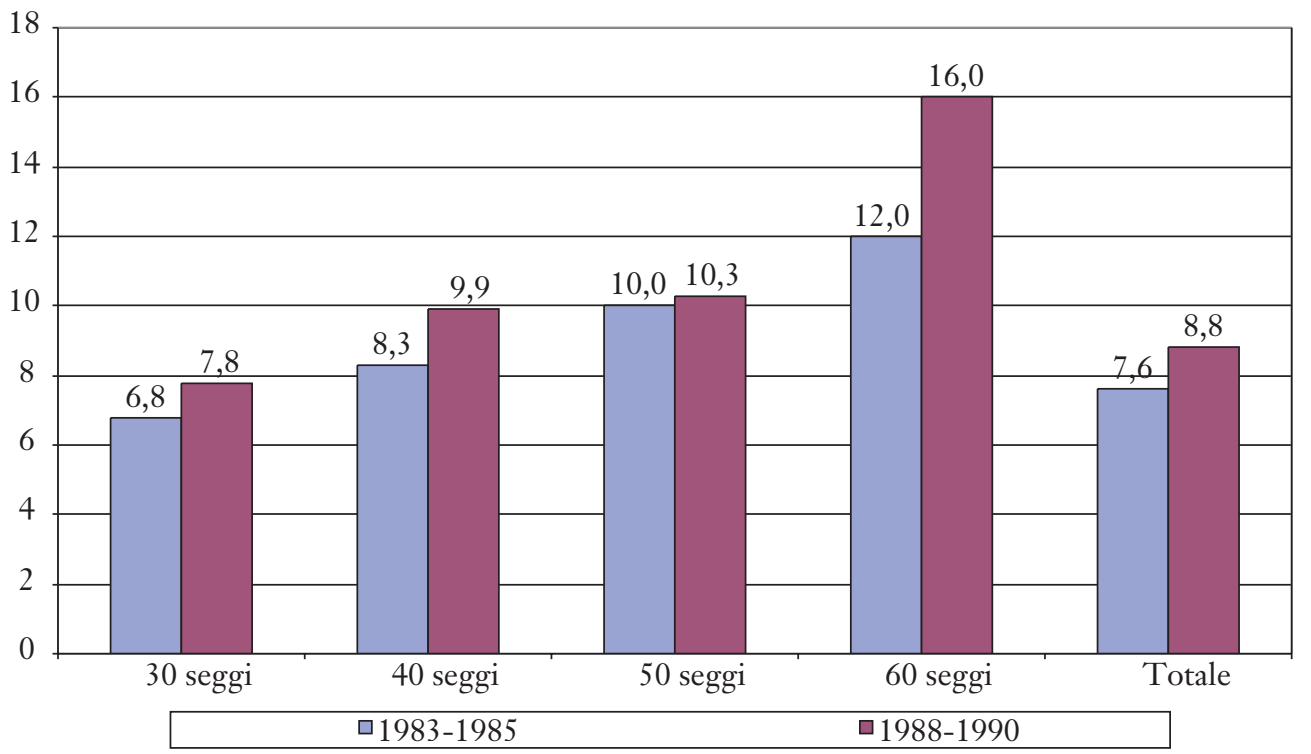


TAB. 11 - Toscana. Numero e media delle liste presentate, per ciclo elettorale e dimensione del consiglio comunale. Comuni con oltre 15 mila abitanti.

\begin{tabular}{llllll}
\cline { 2 - 6 } & \multicolumn{5}{c}{ Ciclo elettorale 1983-1985 } \\
\hline N. seggi Consiglio Comunale & 30 & 40 & 50 & 60 & TOTALE \\
Numero dei Comuni & 31 & 16 & 3 & 1 & 51 \\
Media liste presentate & 6,8 & 8,3 & 10,0 & 12,0 & 7,6 \\
Totale liste presentate & 211 & 133 & 30 & 12 & 386 \\
\hline
\end{tabular}

\begin{tabular}{llllll}
\cline { 2 - 6 } & \multicolumn{7}{l}{ Ciclo elettorale 1988-1990 } \\
\hline N. seggi Consiglio Comunale & 30 & 40 & 50 & 60 & TOTALE \\
Numero dei Comuni & 31 & 16 & 3 & 1 & 51 \\
Media liste presentate & 7,8 & 9,9 & 10,3 & 16,0 & 8,8 \\
Totale liste presentate & 243 & 158 & 31 & 16 & 448 \\
\hline
\end{tabular}

\begin{tabular}{|c|c|c|c|c|c|}
\hline \multirow[b]{2}{*}{ N. seggi Consiglio Comunale } & \multicolumn{5}{|c|}{ Ciclo elettorale 1993-1995 } \\
\hline & 20 & 30 & 40 & 46 & TOTALE \\
\hline Numero dei Comuni & 30 & 11 & 9 & 1 & 51 \\
\hline Media liste presentate & 5,7 & 7,6 & 10,7 & 15,0 & 7,2 \\
\hline \multirow[t]{2}{*}{ Totale liste presentate } & 171 & 84 & 96 & 15 & 366 \\
\hline & \multicolumn{5}{|c|}{ Ciclo elettorale 1997-1999 } \\
\hline N. seggi Consiglio Comunale & 20 & 30 & 40 & 46 & TOTALE \\
\hline Numero dei Comuni & 30 & 11 & 9 & 1 & 51 \\
\hline Media liste presentate & 7,7 & 10,1 & 12,3 & 24,0 & 9,4 \\
\hline \multirow[t]{2}{*}{ Totale liste presentate } & 231 & 111 & 111 & 24 & 477 \\
\hline & \multicolumn{5}{|c|}{ Ciclo elettorale 2000-2004* } \\
\hline N. seggi Consiglio Comunale & 20 & 30 & 40 & 46 & TOTALE \\
\hline Numero dei Comuni & 30 & 12 & 9 & 1 & 52 \\
\hline Media liste presentate & 9,0 & 12,4 & 15,3 & 27,0 & 11,2 \\
\hline Totale liste presentate & 269 & 149 & 138 & 27 & 583 \\
\hline
\end{tabular}

* Nel ciclo 2000-2004, il comune di Pescia ha votato due volte (2001 e 2004): inoltre, i Comuni di Impruneta e Fiesole sono esclusi, in quanto scesi sotto la soglia dei 15 mila ab., mentre sono inclusi Calenzano e Signa, che hanno superato tale soglia demografica. Il Comune di San Giuliano Terme supera i 30 mila ab., e quindi il suo Consiglio Comunale passa da 20 a 30 membri. 
Il primo impatto della nuova legge elettorale, negli anni 1993-1995 (si veda la FIG. 12), ha prodotto in effetti una certa riduzione dell'offerta, ma soprattutto nei comuni più piccoli: nei 30 comuni i cui consigli erano stati ridotti a 20 seggi, la media delle liste presentate si ferma a 5,7; nei comuni con 30 seggi si attestò a 7,6 liste; nei capoluoghi e nelle città maggiori rimase di fatto stabile $(10,7)$ e nella stessa Firenze (dove pure si riduceva notevolmente la posta in gioco, da 60 a 46 seggi), la riduzione risultò modestissima (da 16 a 15 liste).

FIG. 12 - Toscana. Media delle liste presentate, per ciclo elettorale e dimensione del consiglio comunale. Comuni con oltre 15mila abitanti (1993-1995, 1997-1999, 2000-2004).

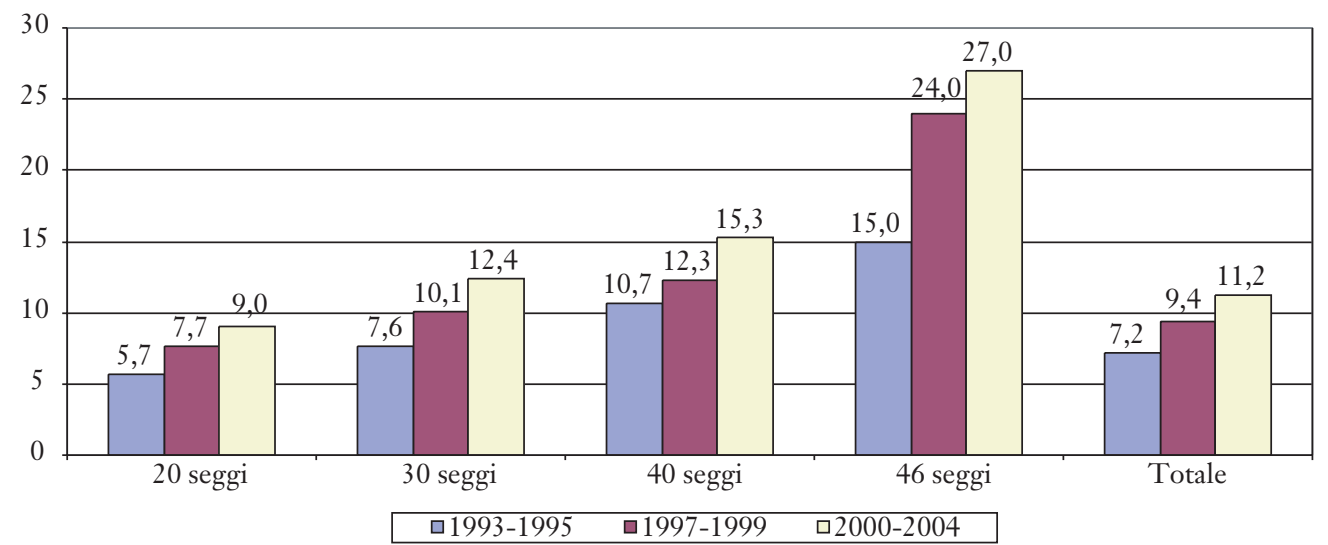

Il ciclo elettorale successivo, tra il 1997 e il 1999, vedeva poi una crescita diffusa e generalizzata delle liste concorrenti: mediamente, da 2 a 3 liste in più, in ciascuna classe demografica (e un ulteriore balzo a Firenze: da 15 a 24 liste). Con il terzo ciclo elettorale, la crescita dell'offerta assume ritmi ancora più incalzanti, giungendo ad una media di 15,3 liste nei comuni con 40 consiglieri; a 12,4 nei comuni medi e a 9,0 nei comuni più piccoli interessati.

Avevamo, dunque, nel complesso, una media di 8,8 liste, nelle ultime elezioni svoltesi con le vecchie regole: ci ritroviamo, alla fine, con una media di 11,2 liste nei comuni che hanno votato tra il 2000 e il 2004; e questo, ricordiamo, nonostante la notevole riduzione dei seggi in palio, pari ad un quarto del totale precedente. Si può dunque concludere che quest'ultima misura, certamente comprensibile e ragionevole, non sembra proprio che abbia prodotto effetti di contenimento della frammentazione in entrata, ossia non abbia sostanzialmente contribuito a limitare e a semplificare l'offerta.

Naturalmente, per analizzare compiutamente le ragioni e le dimensioni di questo fenomeno, occorre anche guardare alla frammentazione in uscita, ossia chiedersi quante delle liste concorrenti siano poi riuscite ad essere rappresentate in consiglio e quale sia stata la "soglia implicita" che ha consentito ad una lista l'accesso in consiglio. I dati mostrano un risultato a prima vista sorprendente, al 
quale occorre trovare una spiegazione (FIG. 13): nelle elezioni pre-riforma, le liste che riuscivano ad entrare nei Consigli comunali corrispondevano a circa i $2 / 3$ delle liste presentate; alla prima prova del nuovo sistema elettorale, come abbiamo appena ricordato, l'offerta di liste si riduceva (da 8,8 a 7,2), ma la percentuale delle liste che riuscivano ad essere rappresentate almeno da un consigliere saliva invece fino a toccare '' $^{\prime} 2 \%$. In valori assoluti, nonostante circa cento liste in meno presentate, il numero delle liste rappresentate restava di fatto invariato (da 314 a 306). Ad una pur modesta limitazione della frammentazione in entrata corrispondeva dunque una situazione immutata nella frammentazione dei Consigli comunali.

FIG. 13 - Toscana. Liste rappresentate nei consigli comunali. Percentuale sulle liste concorrenti, per ciclo elettorale. Comuni con oltre 15 mila abitanti.

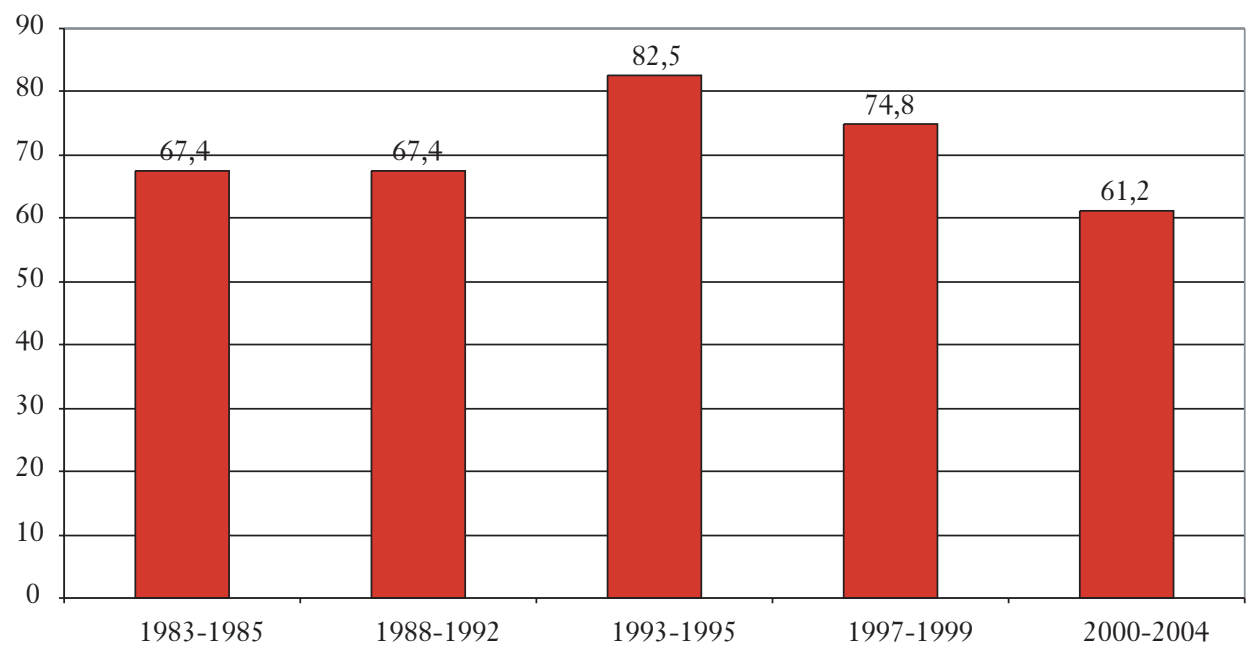

Nel successivo ciclo elettorale (1997-99), torna a crescere anche l'offerta, come se gli attori politici avessero colto nel dato precedente una sorta di incoraggiamento alla corsa elettorale, apparsa meno chiusa di quanto si potesse pensare: se la media delle liste concorrenti sale notevolmente (da 7,2 a 9,4: da 372 a 482 liste), le liste che passano sono ancora il $74,8 \%$, cioè rimane una percentuale di successo molto elevata, solo poco inferiore a quella precedente. I segni di una possibile svolta giungono con il terzo ciclo elettorale: se, come abbiamo visto, la frammentazione in entrata continua a crescere, quella in uscita segna un deciso e significativo arretramento e, per la prima volta, si giunge ad una percentuale di accesso inferiore a quella degli anni Ottanta, con il 61,2 \% delle liste presentate che riescono ad accedere ai consigli.

Come interpretare questi fenomeni e anche questa possibile inversione di tendenza? La risposta non può che essere cercata nelle caratteristiche stesse del nuovo sistema elettorale e, in particolare, in quello che possiamo definire il combinarsi e sovrapporsi delle diverse arene competitive. Come sappiamo, il voto alle liste, ai 
fini dell'elezione dei consiglieri, si intreccia a quello per i candidati sindaci e la possibilità di una pluralità di opzioni, nelle modalità di voto concesse all'elettore, incide sull'intera dinamica della competizione elettorale e sugli effetti che essa produce. In particolare, il meccanismo del voto trasferito automaticamente dalle liste ai candidati (quando l'elettore non esprime un voto ai candidati sindaco), è quello che va messo sotto osservazione, come indiziato principale della mancata riduzione della frammentazione nell'offerta elettorale. Una possibile interpretazione del fenomeno va ricondotta cioè agli incentivi impliciti che il meccanismo elettorale prevede e che inducono i protagonisti principali che agiscono sulla scena, ossia i candidati alla carica di sindaco, a puntare su una ricca e ampia costellazione di liste attorno alla propria candidatura, proprio per potersi meglio avvalere del meccanismo di trasferimento del voto. L'assenza di una soglia di sbarramento, per le liste dentro coalizioni ampie, contribuisce poi, per la sua parte, ad incentivare un'ampia offerta di liste a sostegno dei maggiori candidati alla carica di sindaco.

Questo fenomeno, come abbiamo visto, continua anche con il terzo e più recente ciclo elettorale, salvo arrestarsi decisamente la quota di liste che riescono a raccogliere i consensi sufficienti a conquistare almeno un seggio. Come spiegare questo cambiamento? Una possibile risposta va ricercata nella possibilità che cominci ad emergere una diversa e più selettiva risposta degli elettori e che la sovrabbondanza dell'offerta di liste (e di micro-liste) abbia cominciato a produrre una sorta di fenomeno di rigetto; i sindaci continuano ad incentivare o quanto meno ad accettare la presentazione di molte liste a sostegno, ma evidentemente si è toccata una soglia critica, oltre la quale l'"eccesso" di concorrenzialità (anche interna alle coalizioni) non può che produrre una maggiore selezione. Inoltre, e questo per la Toscana vale particolarmente, è certamente da mettere in conto, come altra possibile spiegazione, che il sistema dei partiti stia recuperando, rispetto al decennio scorso, un grado maggiore di solidità, scoraggiando spinte centrifughe o localistiche.

Nel complesso, dunque, siamo pienamente dentro quella logica di «bipolarismo frammentato» che caratterizza l'intero percorso riformatore delle leggi elettorali italiane, nella prima metà degli anni Novanta: una sorta di compromesso tra le esigenze di stabilità, di personalizzazione e di responsabilizzazione degli esecutivi, da una parte; e l'accettazione, il riconoscimento e la riproduzione della perdurante frammentazione del sistema dei partiti e delle coalizioni, dall'altra. Una logica non priva di conseguenze sull'intero assetto politico ed istituzionale dei governi locali, all'interno dei quali viene a crearsi una peculiare combinazione tra la nuova centralità e visibilità della figura del sindaco, legittimato dal voto popolare e particolarmente esposto quindi alla pluralità e varietà della domanda politica e delle aspettative provenienti dal basso (con pochi filtri selettivi, stante la debolezza e la crisi dei partiti), e una sfera della rappresentanza, nei consigli, comunque frammentata e spesso protesa alla vana (e talvolta distorta e impropria) ricerca di un ruolo perduto. 
Candidati e liste. - Nei Comuni toscani che stiamo analizzando l'evoluzione dell'offerta elettorale ha avuto un andamento differenziato: mentre il numero delle liste concorrenti cresce notevolmente, il numero dei candidati sindaco rimane sostanzialmente stabile (si vedano la FIG. 14 e, più avanti, la TAB. 14). È un dato coerente con le possibili interpretazioni, sopra ricordate, circa le ragioni che incentivano invece una frammentazione dal versante dell'offerta di rappresentanza delle liste, con la tendenza ad aggregare coalizioni quanto più ampie possibili, nel contesto della peculiare dinamica bipolare che caratterizza il nostro sistema politico.

FIG. 14 - Toscana. Media dei candidati e media delle liste presentate, per ciclo elettorale. Comuni con oltre 15mila abitanti.

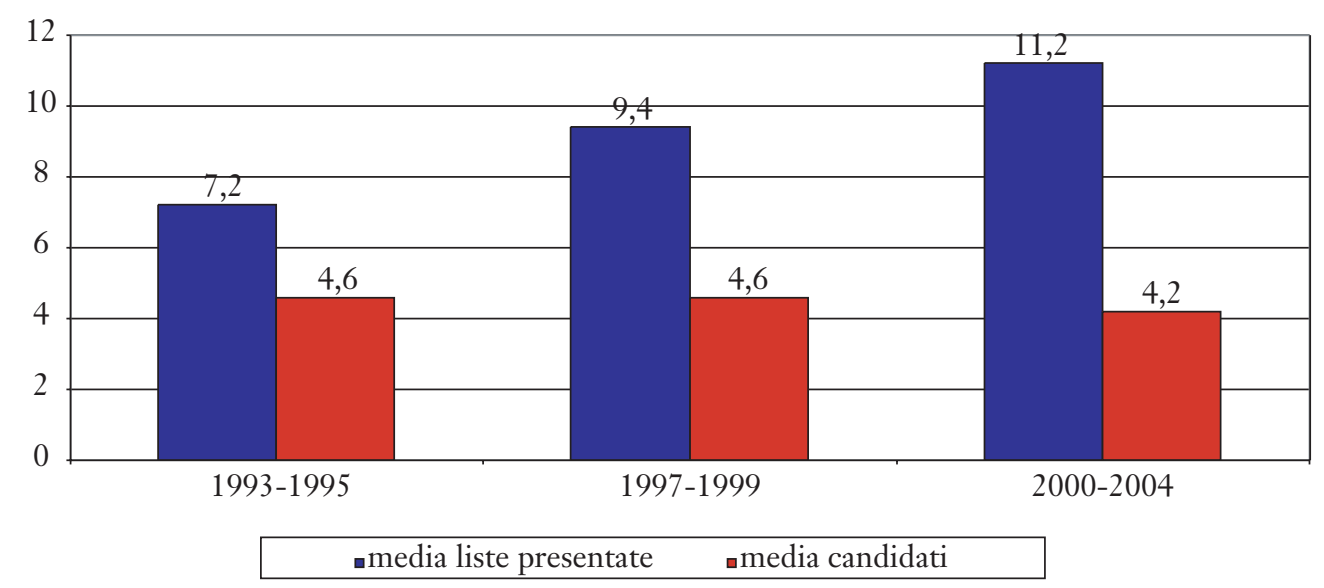

Sono le due coalizioni maggiori, ovviamente, a fornire il maggiore apporto alla proliferazione di liste: è ad esse che va attribuito lo scarto tra il numero (stabile) dei candidati sindaco e quello (crescente) delle liste di supporto. Se guardiamo infatti al rapporto tra candidati e liste, notiamo che, in generale (FIGG. 15 e 16), nel 1993-1995, oltre due terzi dei candidati aveva a proprio sostegno una sola lista; ma se guardiamo solo ai candidati eletti, erano solo il $21 \%$ i candidati eletti ad avere il supporto di una sola lista. Nella successiva tornata elettorale, la forbice aumenta: in generale, i candidati con una sola lista a sostegno sono ancora oltre il $60 \%$; ma, al contrario, i sindaci eletti con una sola lista sono stavolta appena il $4 \%$ del totale, mentre come ben si vede dalla FIG. 16, sono molto più frequenti i casi con almeno 4 liste collegate. Questo "slittamento", nella distribuzione della frequenza delle possibili combinazioni tra candidati vincenti e numero di liste collegate, riflette il profondo mutamento intervenuto nella seconda metà degli anni Novanta: se ancora con il primo ciclo elettorale, $2 / 3$ dei candidati avevano a sostegno da una sola lista ad un massimo di tre, e solo il restante terzo dei candidati eletti ne registrava da 4 a 8 , con il successivo ciclo elettorale, il quadro cambia radicalmente. Spariscono, o quasi, le liste unitarie di coalizione, cresce il numero di liste a sostegno dei candidati, i partiti sembrano puntare sempre più su una pro- 
pria autonoma presenza nelle coalizioni. Nel 1997-1999, due terzi dei candidati hanno tra le 4 e le 7 liste di sostegno, con una situazione esattamente rovesciata, rispetto a quattro anni prima. Con il terzo ciclo elettorale (2000-2004), la tendenza si fa ancora più vistosa: non ci sono più candidati vincenti con una sola lista a sostegno, mentre quasi due su tre dei "vincenti" hanno tra 5 e 7 liste.

FIG. 15 - Toscana. Percentuale di candidati, secondo il numero di liste collegate. Comuni con oltre 15 mila abitanti.

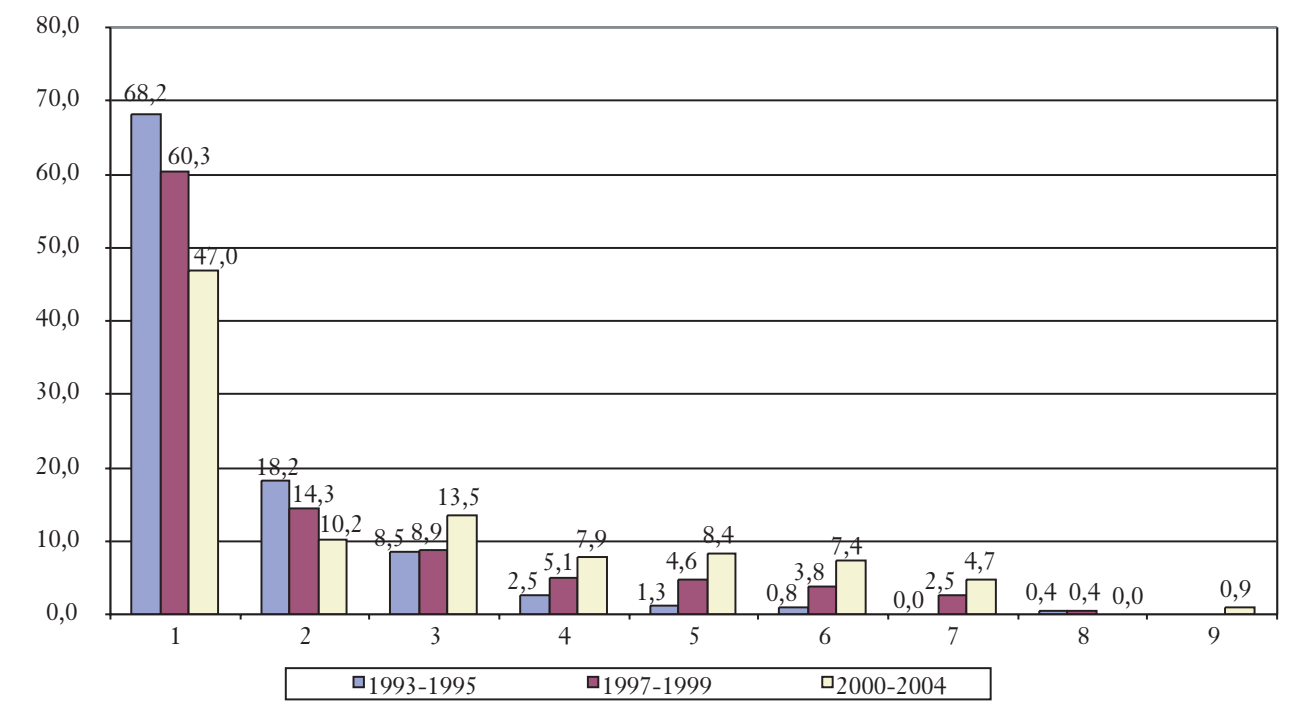

FIG. 16 - Toscana. Percentuale di candidati vincenti, secondo il numero di liste collegate. Comuni con oltre 15 mila abitanti.

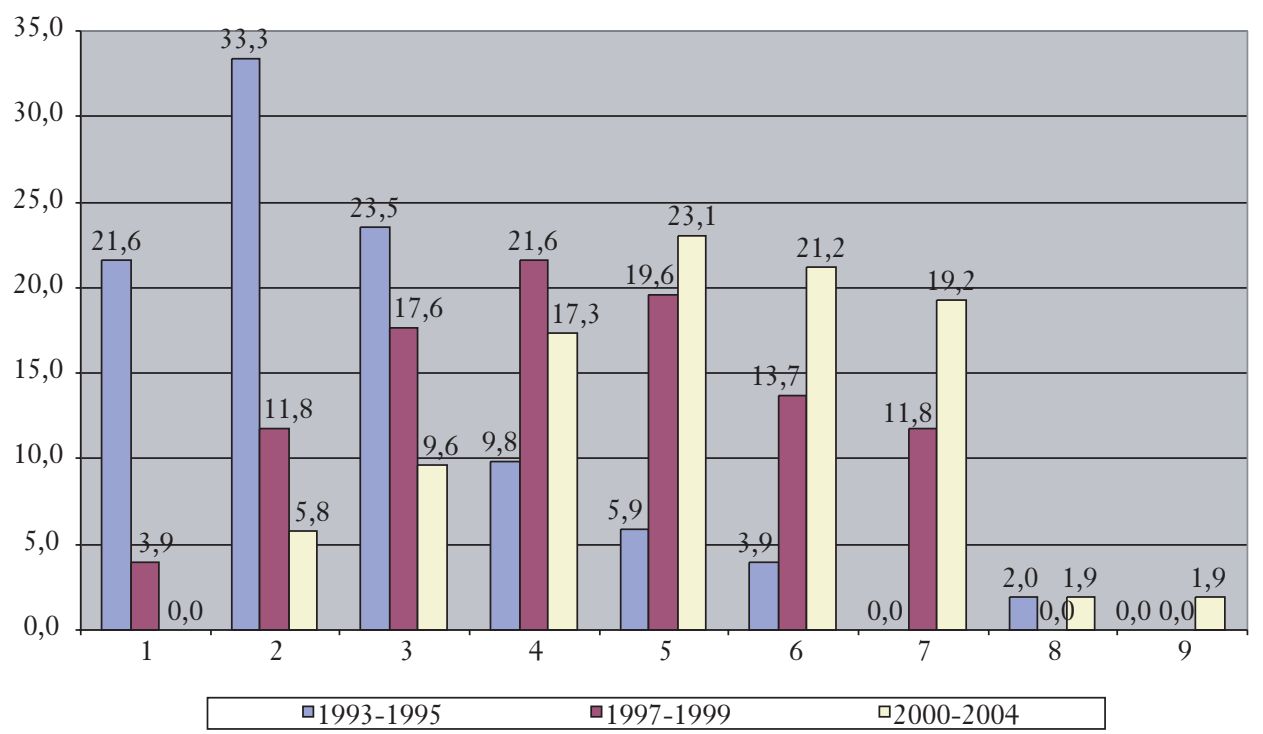


Riassumendo, se nel 1993-1995 la media delle liste a sostegno dei sindaci eletti era di 2,7, nel 1997-1999 questa media sale a 4,3, mentre nei comuni che hanno votato nel 2000-2004 sale ulteriormente sino a 5,2 (FIG. 17).

FIG. 17 - Toscana. Media delle liste a sostegno dei candidati vincenti, per classi dimensionali dei consigli comunali. Comuni con oltre 15 mila abitanti.

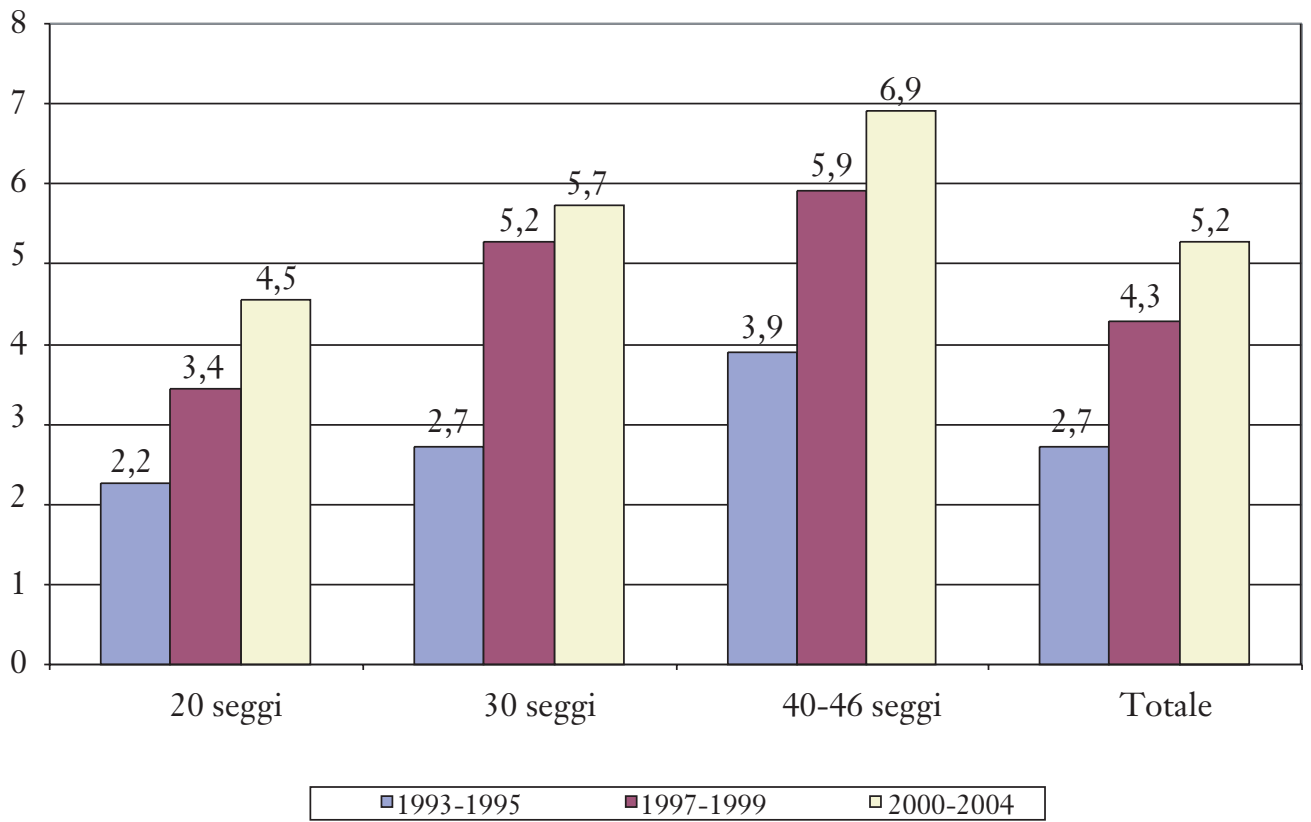

Possiamo concludere, dunque, che il meccanismo di assegnazione del premio di maggioranza, unito alla norma sulla soglia differenziata di sbarramento, ha prodotto un effetto di incremento della frammentazione che ha di fatto compensato gli effetti di riduzione indotti dalla minore ampiezza delle assemblee da eleggere.

Insieme a questo processo, se ne è sviluppato un altro, che conferma la crescente specificità del voto amministrativo e la peculiare accentuazione che il nuovo sistema elettorale ha impresso alle stesse forme dell'offerta politica locale. La specificità della competizione locale può essere colta grazie ad un'analisi della particolare tipologia delle liste presentate: possiamo chiederci, cioè, quale sia stata la natura e il profilo di queste liste ed in che misura i sistemi politici locali sembrano allinearsi al formato dello scenario politico nazionale. Abbiamo costruito, a tal fine, una classificazione delle liste secondo la loro natura nazionale o locale e 
secondo il loro essere interne o esterne alle due maggiori coalizioni, presenti in coalizione o isolate ${ }^{25}$, con la seguente griglia:

\begin{tabular}{ll}
\hline Tipo & Descrizione \\
\hline Liste A & Partiti nazionali, o simboli nazionali di coalizione \\
Liste B & Liste locali, con simbolo locale, non appartenenti ad alcuna coalizione \\
Liste C & Liste locali di coalizione tra partiti nazionali, con simbolo locale e riferimento "civico" \\
Liste D & $\begin{array}{l}\text { Liste locali, con simbolo locale e riferimento "civico", appartenenti ad una coalizione } \\
\text { nazionale }\end{array}$ \\
Liste E & Liste locali non coalizzate, ma senza denominazioni, simboli o riferimenti "civici" \\
\hline
\end{tabular}

Come emerge dalla FIG. 18, se prima della riforma, il contesto della competizione locale era in larghissima misura coincidente con quello dello scenario politico nazionale, dopo la riforma la natura delle liste si fa più variegata, rispondendo a logiche diverse. Negli anni Ottanta, fino al turno elettorale del 1990, il 95\% delle liste presentate erano liste di partito, con la denominazione e il simbolo nazionale del partito: le liste civiche locali erano residuali, mentre praticamente assenti erano le stesse liste di coalizione tra i partiti nazionali (e se ne comprende bene il motivo, data la natura proporzionale del sistema elettorale). Il momento acuto di crisi e di destrutturazione del sistema dei partiti e, nello stesso tempo, il primo impatto della riforma elettorale, si coglie bene nei dati relativi al primo ciclo elettorale svoltosi con le nuove regole: nel 1993-1995, le liste nazionali scendono decisamente, fino ai $2 / 3$ del totale, mentre un quinto sono liste locali di coalizione tra i partiti (un dato coerente con quello visto in precedenza, relativo al numero di candidati vincenti con una sola lista a sostegno); crescono, inoltre, le liste locali isolate $(6,5 \%)$ e fanno la loro comparsa, con il $5,4 \%$ (essendo prima escluse dalla logica stessa del sistema elettorale) anche le liste civiche o locali appartenenti alle coalizioni maggiori. Si tratta, in quest'ultimo caso, di un fenomeno che ha avuto una certa risonanza, soprattutto in relazione al voto di alcune grandi città, e che è stato ricondotto ad un'espressione di ulteriore personalizzazione della competizione (le «liste del sindaco»): in realtà, nella generalità dei casi, specie quando si tratta di città medio-piccole, non sembra molto rilevante la presenza di liste interpretabili come proiezione diretta e personale del candidato sindaco, costruite dal candidato sindaco o che ad esso si richiamano apertamente anche nella denominazione adottata; la presenza di liste civiche all'interno delle coalizioni maggiori può essere piuttosto vista come espressione di gruppi locali che cercano, in tal modo, di rappresentare particolari istanze o interessi della

25 Si tratta di una classificazione che, ovviamente, in qualche caso, ci ha costretto ad una qualche forzatura interpretativa: ad esempio, ed è stato il caso più frequente, una delle due coalizioni spesso si divide in due tronconi, ciascuno dei quali composto da più partiti di profilo nazionale. In questi casi, abbiamo comunque considerato in primo luogo la loro natura di liste "nazionali". 
società locale e, soprattutto, di contribuire in modo determinante all'elezione di un sindaco, conquistando così un proprio spazio contrattuale all'interno della coalizione. D'altra parte, come si ricordava sopra, è insito nella stessa logica del meccanismo elettorale una sorta di convenienza sistemica, da parte dei candidati sindaco, ad allargare la propria coalizione e a sfruttare quanto più possibile il meccanismo di "trasferimento" automatico del voto, nel caso in cui un elettore si limiti ad esprimere un voto di lista (tra l'altro, spesso, trascinato a sua volta dal voto di preferenza espresso ad un candidato consigliere).

FIG. 18 - Toscana. Tipologia delle liste presentate, per cicli elettorali (valori percentuali). Comuni con oltre 15 mila abitanti.

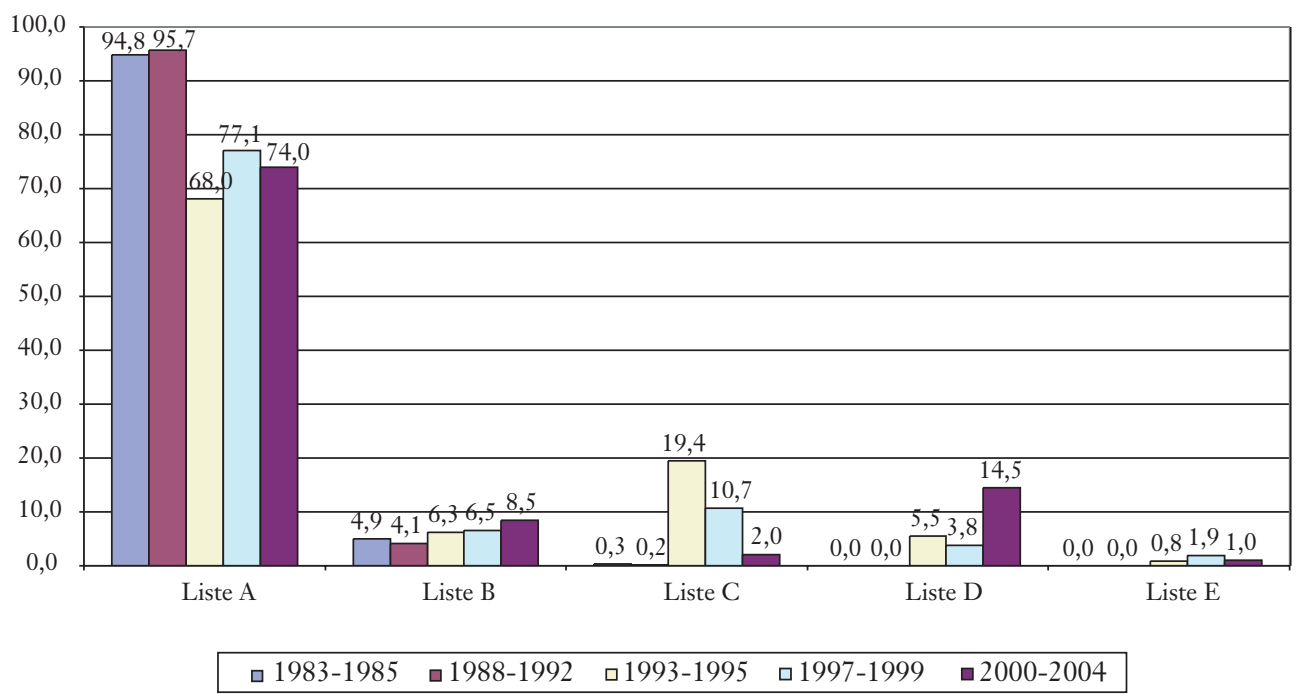

Liste A Partiti nazionali, o simboli nazionali di coalizione

Liste B Liste locali, con simbolo locale, non appartenenti ad alcuna coalizione

Liste C Liste locali di coalizione tra partiti nazionali, con simbolo locale e riferimento "civico"

Liste D Liste locali, con simbolo locale e riferimento "civico", appartenenti ad una coalizione nazionale

Liste E Liste locali non coalizzate, ma senza denominazioni, simboli o riferimenti "civici"

Le elezioni del 1997-99 e poi quelle del 2000-04, segnano in una certa misura il ritorno dei partiti, le cui liste risalgono intorno ai tre quarti del totale: ma probabilmente, non è corretto accreditare quella lettura (in chiave di "antipolitica") che pure, da più parti, negli anni scorsi, è stata avanzata, secondo la quale, passato il primo momento di entusiasmo e di partecipazione della società civile, anche le elezioni locali sono state nuovamente preda dell'invadenza dei partiti. In realtà, come mostra bene la FIG. 18 (si veda l'andamento delle liste C), non sono le liste della cosiddetta società civile ad eclissarsi: la maggior presenza di liste di partito nasce dalla ridotta presenza di liste unitarie di coalizione, praticamente scom- 
parse poi con il terzo ciclo elettorale. Una conferma, quindi, della natura delle coalizioni come cartelli elettorali, tra partiti che sembrano tenere molto alla propria identità e che non rinunciano ad esprimerla, specie quando lo stesso sistema elettorale prevede meccanismi tali da premiare la distinzione e la visibilità dei vari attori. Una logica che viene poi confermata dall'altro dato emergente dall'ultima tornata elettorale: il fenomeno delle liste locali interne alle coalizioni maggiori assume proporzioni ancora più vistose, fino a toccare il 14,5\% del totale delle liste presentate; ma appare quanto meno dubbio accreditare questo tipo di liste di una qualche peculiare valenza antipartitocratica, essendo in gran parte semmai riconducibili alle logiche contrattuali di alcuni specifici segmenti del ceto politico locale.

\section{Il formato dei sistemi politici locali}

Soglie legali e soglie di fatto. - Gli effetti delle nuove regole elettorali sulle forme e i livelli della rappresentanza possono essere analizzati anche da un altro punto di osservazione, quello della soglia implicita di accesso ai Consigli comunali, ossia la percentuale minima di voto che si è rivelata necessaria, mediamente, perché una lista riuscisse a conquistare almeno un seggio.

Guardiamo innanzi tutto alla situazione negli anni Ottanta (FIG. 19), osservando quale fosse allora la percentuale di voto più bassa che consentiva ad una lista la conquista di almeno un consigliere: tale soglia si collocava mediamente al 3,2\%, ma nei comuni maggiori tale soglia era ancora più bassa, bastando il 2,0-2,3\% per ottenere almeno un seggio; mentre nei comuni più piccoli tale soglia di fatto si innalzava al 3,6-3,7\%. Il metodo proporzionale, con il divisore D'Hondt, limitava in qualche modo un eccesso di frammentazione nei Consigli: effetto, peraltro, ottenuto anche grazie al formato standard del sistema dei partiti della Prima Repubblica, che si riproduceva su scala locale: i sei partiti dell'arco costituzionale, più il MSI, più i Verdi (che cominciano a presentarsi localmente con più frequenza nella seconda metà degli anni Ottanta); più, in qualche caso, una formazione di estrema sinistra.

Con il nuovo sistema elettorale, e poi la soglia legale introdotta nel 1999, la situazione cambia e impone anche una lettura differenziata, distinguendo tra la misurazione di una soglia relativa alle liste che facciano parte di una coalizione vincente, e che si avvalgono dell'eventuale premio di maggioranza e la soglia che invece si riferisce alla liste di minoranza ${ }^{26}$.

${ }^{26}$ Va ricordato, inoltre, come la legge preveda, in prima istanza, nel caso di elezione al primo turno di un sindaco, una verifica sulla distribuzione proporzionale dei seggi effettuata mediante metodo proporzionale (divisore D'Hondt): se le liste collegate al sindaco eletto raggiungono già il $60 \%$ dei seggi, non scatta alcun premio di maggioranza (un caso, peraltro, piuttosto frequente in Toscana). Nella nostra analisi, per evidenti ragioni di semplificazione, abbiamo comunque distinto tra la soglia di accesso al consiglio comunale delle liste collegate al sindaco eletto e quella delle altre liste. 
Fig. 19 - Toscana. Soglia media di accesso ai consigli comunali. Percentuale media di voto, per classi dimensionali dei consigli comunali (1983-1990). Comuni con oltre 15 mila abitanti.

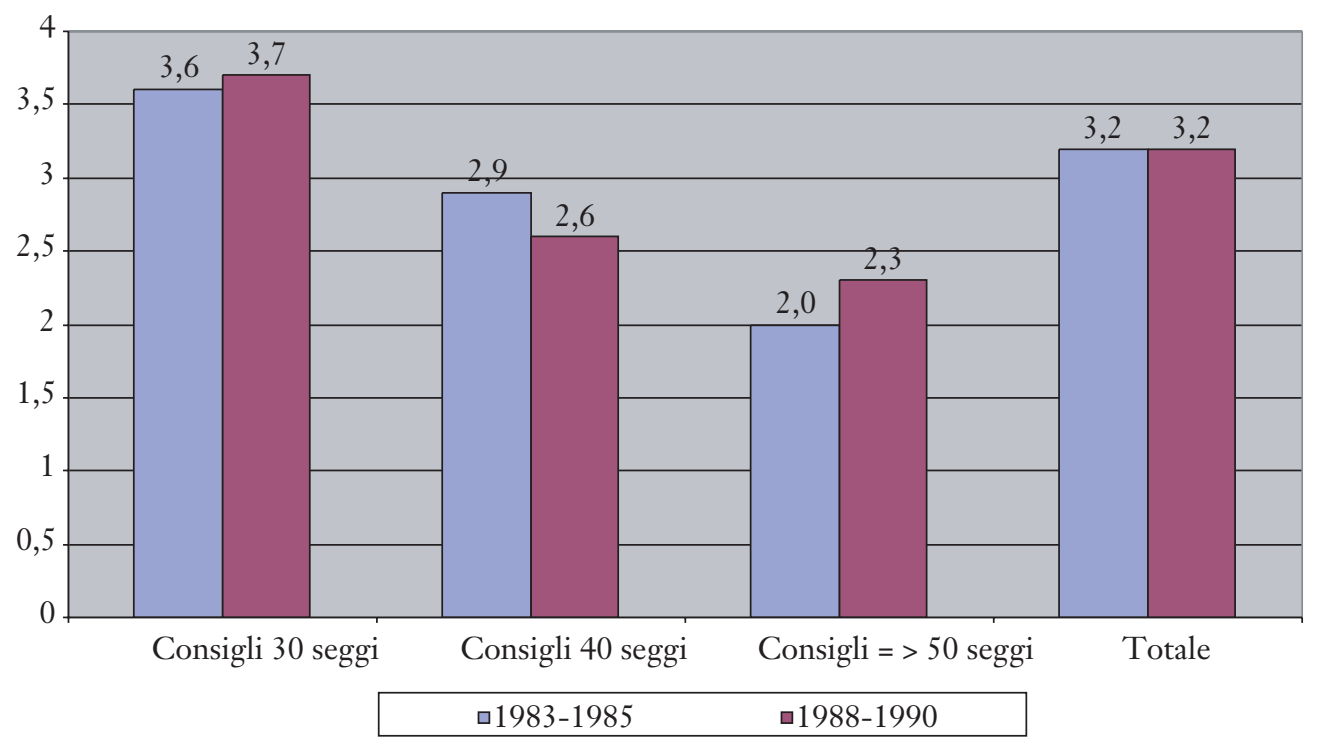

Tra il prima e il dopo riforma si nota in effetti un innalzamento della soglia di fatto, che però assume dimensioni significative soprattutto per le liste collegate ai candidanti perdenti (FIG. 20): nel complesso, rispetto ad una soglia media del $3,2 \%$, nelle elezioni pre-riforma, si passa successivamente, per le liste collegate al sindaco eletto ad una soglia del 3,9\% (1993-95) e del 4,3\% (1997-99 e 20002004), mentre per tutte le altre liste, collegate ai candidati perdenti, si passa ad una soglia decisamente più elevata $(5,6 \%$, nel primo ciclo elettorale; $4,7 \%$ nel secondo ciclo elettorale; $5,4 \%$ nel terzo ciclo elettorale).

L'altro dato che sembra caratterizzare gli effetti di questo sistema elettorale sulle soglie implicite di accesso è dato da una più accentuata rilevanza della variabile dimensionale dei Consigli (FIG. 21 e TAB. 12): per i comuni maggiori, e per le liste collegate al sindaco eletto, siamo sostanzialmente allo stesso livello della soglia pre-riforma; mentre solo per i comuni più piccoli, per i quali ha pesato di più la riduzione dell'ampiezza dell'assemblea, l'effetto di innalzamento della soglia appare più rilevante (ma anche in questo caso, evidentemente, soprattutto per le liste perdenti). Se confrontiamo ad esempio i comuni con 50 e più consiglieri del periodo pre-riforma, e i comuni con 40 e più consiglieri del periodo post-riforma, la soglia di accesso per le liste vincenti è praticamente uguale $(2,1-2,6 \%$, rispetto al $2,0-2,3 \%$ del periodo precedente); mentre è appena più elevata per le altre liste. 
Fig. 20 - Toscana. Soglia media di accesso ai consigli comunali. Percentuale di voto più bassa tra le liste che ottengono almeno un seggio. Comuni con oltre 15 mila abitanti.

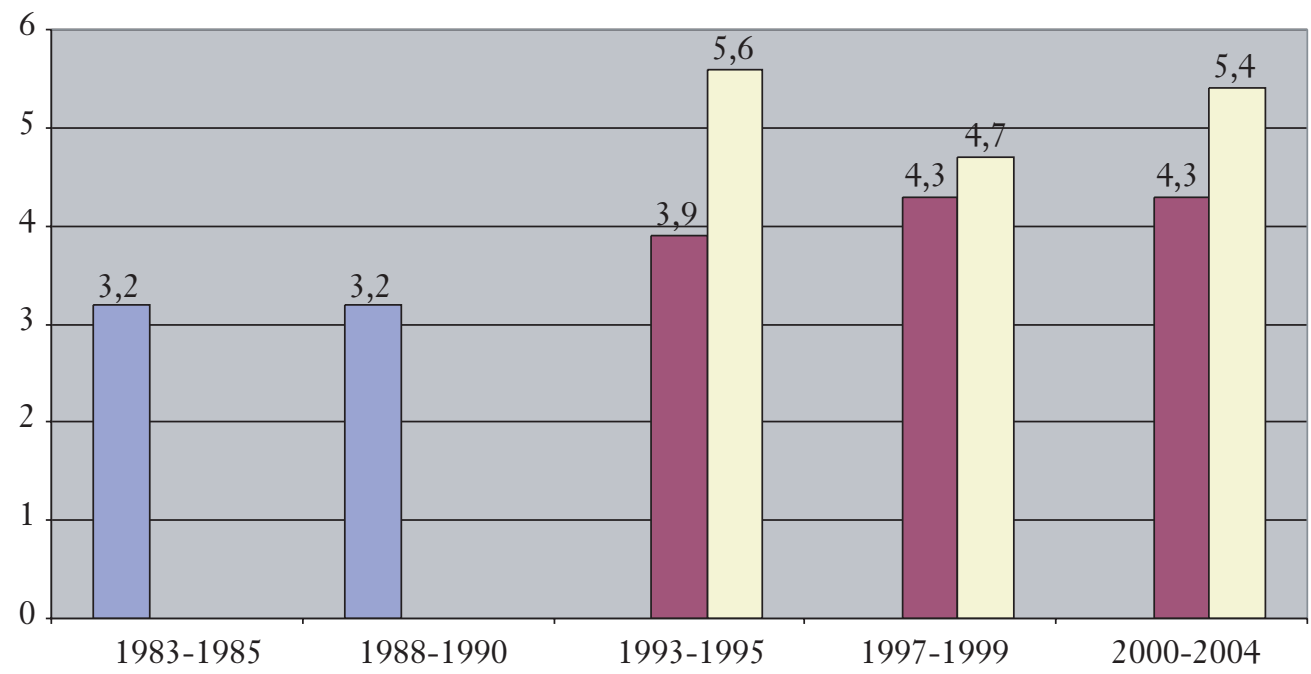

$\square$ Totale

$\square$ liste vincenti

$\square$ liste perdenti

FIG. 21 - Toscana. Soglia media di accesso ai consigli comunali, per classi dimensionali e per tipo di lista. Comuni con oltre 15 mila abitanti.

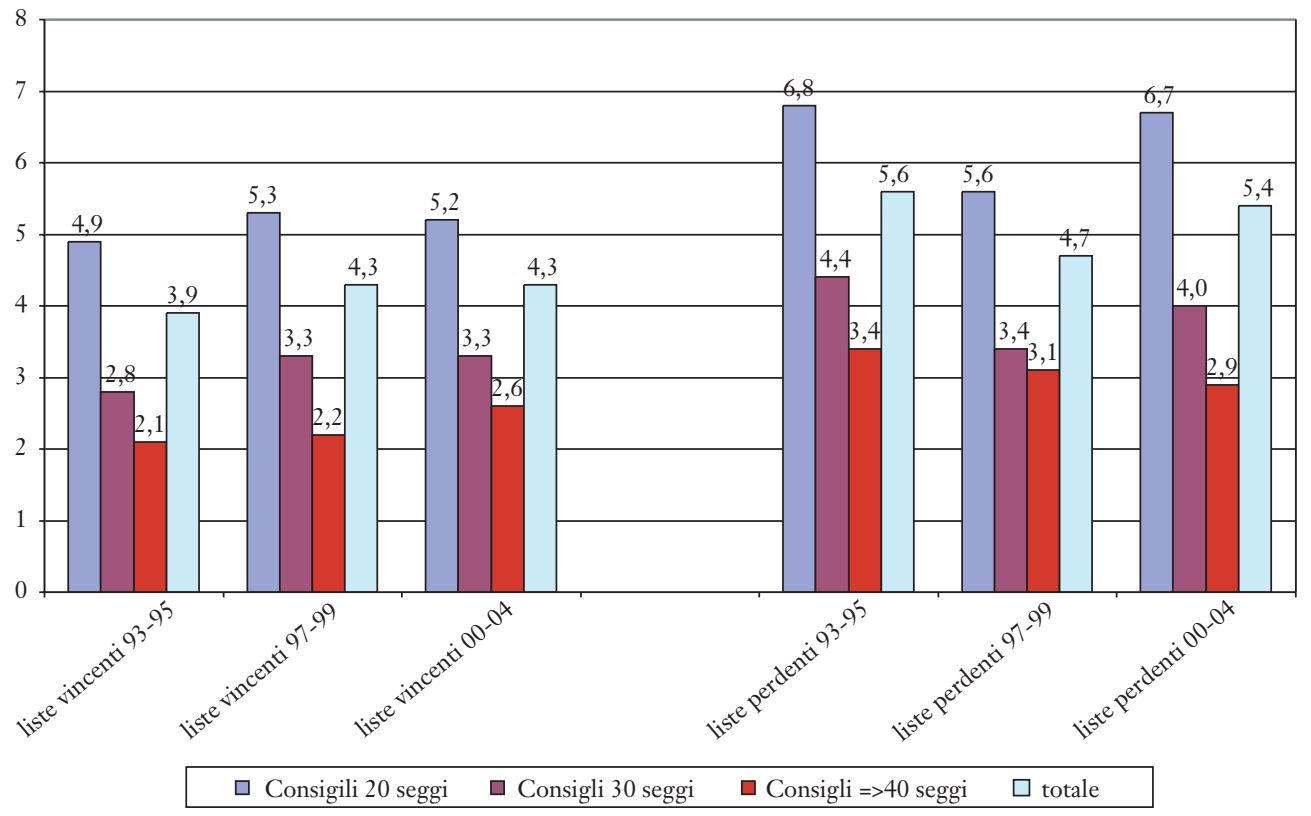


TAB. 12 - Toscana. Soglia media di accesso ai consigli comunali. Percentuale media di voto alle liste con la percentuale di voto più bassa, che riescono ad ottenere almeno un seggio.

\begin{tabular}{cccccccccc}
\hline Ampiezza & $1983-$ & $1988-$ & Ampiezza & \multicolumn{2}{c}{$1993-1995$} & \multicolumn{1}{c}{$1997-1999$} & \multicolumn{2}{c}{$2000-2004$} \\
\cline { 5 - 10 } $\begin{array}{c}\text { Consigli } \\
\text { (n. seggi) }\end{array}$ & 1985 & 1990 & $\begin{array}{c}\text { Consigli } \\
\text { (n. seggi) }\end{array}$ & A & B & A & B & A & B \\
\hline 30 & 3,6 & 3,7 & 20 & 4,9 & 6,8 & 5,3 & 5,6 & 5,2 & 6,7 \\
40 & 2,9 & 2,6 & 30 & 2,8 & 4,4 & 3,3 & 3,4 & 3,3 & 4,0 \\
$>50$ & 2,0 & 2,3 & $>40$ & 2,1 & 3,4 & 2,2 & 3,1 & 2,6 & 2,9 \\
\hline Totale & 3,2 & 3,2 & Totale & 3,9 & 5,6 & 4,3 & 4,7 & 4,3 & 5,4 \\
\hline
\end{tabular}

$A=$ liste collegate al sindaco eletto.

$B=$ liste collegate agli altri candidati sindaci.

Dimensioni e misure del bipolarismo. - Alla fine di questo paragrafo, possiamo tuttavia chiederci: i dati fin qui analizzati, soprattutto quelli relativi alle liste concorrenti e alle liste rappresentate, esprimono effettivamente un livello elevato di frammentazione dei sistemi politici locali? Ossia, è possibile distinguere tra i candidati e le liste che risultano effettivamente rilevanti, nello strutturare i sistemi politici locali, e i candidati e le liste che, per la loro forza elettorale, si rivelano marginali? È possibile misurare il grado effettivo di bipolarismo e il grado effettivo di frammentazione della rappresentanza elettorale (cioè dei voti espressi) e consiliare (cioè dei seggi ottenuti)?

A questa domanda si può rispondere analizzando dapprima l'indice di bipolarismo, ossia la somma delle percentuali ottenute dai primi due candidati e dalle prime due liste, nelle varie consultazioni. L'indice di bipolarismo relativo ai candidati non lascia molti dubbi interpretativi: se negli anni 1993-1995, tale indice si attestava mediamente al 78,5\% (con un dato leggermente inferiore nei comuni maggiori), quattro anni dopo esso cresceva fino a toccare l' $85,3 \%$ (si vedano la FIG. 22 e la TAB. 13) e salire ancora all' $87,2 \%$ nel ciclo elettorale più recente. Anche i sistemi politici locali della Toscana, dunque, tendono a strutturarsi secondo una logica bipolare che sembra accentuarsi nel corso del decennio. Possiamo definirlo, tuttavia, come un bipolarismo un po' monco, «a candidato dominante» (Sani): nel primo ciclo, infatti, la media dei voti riportata al primo turno dal candidato primo classificato è stata pari al 53,4\%, mentre quella del secondo si fermava al 25,1\%; nel secondo ciclo, tali valori salgono, rispettivamente, fino al $57,3 \%$ e al $28,1 \%$, con una maggiore polarizzazione, che però non attenua l'entità media dello scarto tra i primi due contendenti; fenomeno che si accentua con il ciclo elettorale 2000-2004, quando i primi ottengono mediamente ancora di più $(59,1 \%)$ e i secondi restano fermi al $28,1 \%$. Le dimensioni di tale scarto, peraltro, sono ancora maggiori nei centri più piccoli (si veda la TAB. 13).

Non si tratta, dunque, di un bipolarismo "perfetto", e non solo per questo netto squilibrio competitivo tra i primi due candidati: significativo appare comunque il fatto che agli altri candidati (e ricordiamo che la media di candidati in corsa è stata di 4,6) rimane pur sempre una quota di consensi elettorali intorno al 13- 
$15 \%$. Ai margini o ai confini delle due coalizioni maggiori, vi è una parte di elettori che tende a rifiutare la dimensione bipolare della competizione, ma anche, e innanzi tutto, una parte di ceto politico locale che probabilmente tenta di giocare una propria partita negli interstizi di questa competizione, (magari in chiave contrattuale, puntando sulla possibilità offerte in questo senso dal sistema a doppio turno). Va poi considerato che, in molti casi, gli altri candidati sono pur sempre riconducibili ad uno dei due maggiori schieramenti e che la loro corsa separata nasce spesso da tensioni e difficoltà nei rapporti infracoalizionali su scala locale.

FIG. 22 - Toscana. Indice di bipolarismo dei candidati, per classi dimensionali del consiglio comunale e per ciclo elettorale. 51 comuni con oltre 15 mila abitanti.

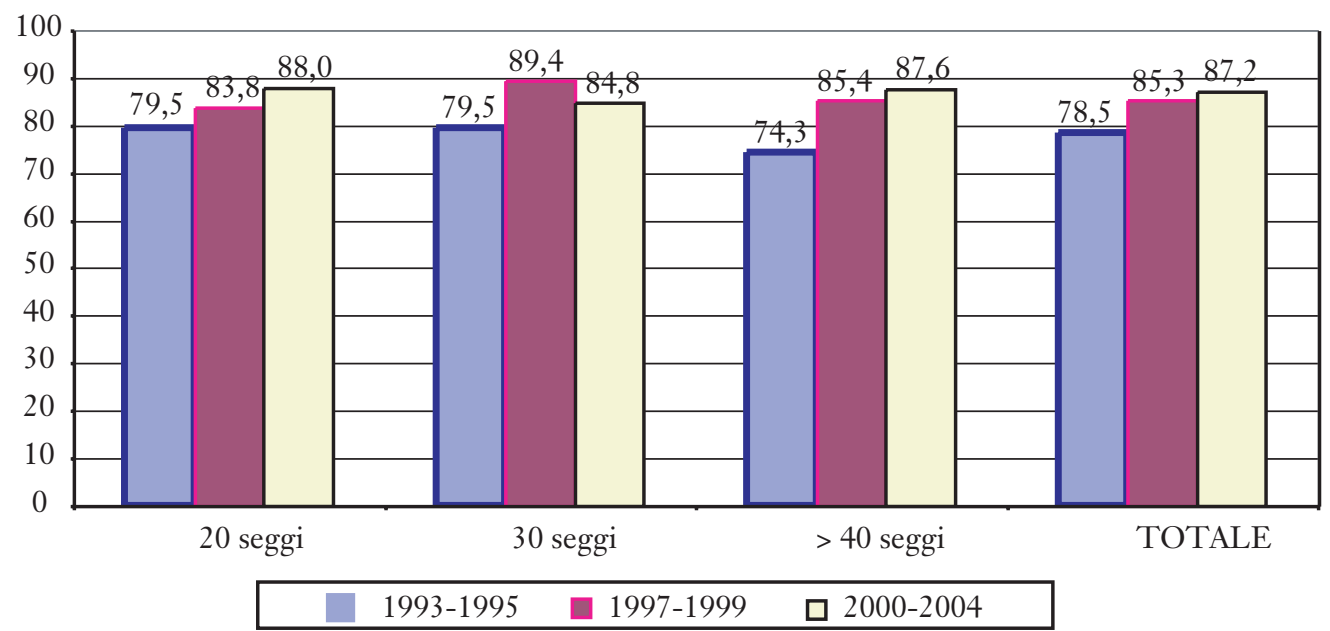

TAB. 13 - Toscana. Indice di bipolarismo. Comuni con oltre 15 mila abitanti.

\begin{tabular}{|c|c|c|c|c|c|}
\hline $\begin{array}{c}\text { Dimensione } \\
\text { del consiglio comunale } \\
\text { (n. seggi) }\end{array}$ & N. di comuni & Ciclo elettorale & $\begin{array}{l}\text { Voti primo } \\
\text { candidato } \\
\text { (\% media) }\end{array}$ & $\begin{array}{l}\text { Voti secondo } \\
\text { candidato } \\
\text { (\% media) }\end{array}$ & $\begin{array}{c}\text { Indice di } \\
\text { bipolarismo }\end{array}$ \\
\hline & & 1993-1995 & & & \\
\hline 20 & 31 & & 55,2 & 24,3 & 79,5 \\
\hline 30 & 10 & & 53,8 & 25,6 & 79,5 \\
\hline$>40$ & 10 & & 45,9 & 28,4 & 74,3 \\
\hline \multirow[t]{2}{*}{ Totale } & 51 & & 53,1 & 25,4 & 78,5 \\
\hline & & 1997-1999 & & & \\
\hline 20 & 30 & & 56,4 & 27,5 & 83,8 \\
\hline 30 & 10 & & 63,3 & 26,2 & 89,4 \\
\hline$>40$ & 10 & & 53,1 & 32,3 & 85,4 \\
\hline \multirow[t]{2}{*}{ Totale } & 51 & & 57,2 & 28,1 & 85,3 \\
\hline & & $2000-2004$ & & & \\
\hline 20 & 30 & & 60,5 & 27,5 & 88,0 \\
\hline 30 & 12 & & 58,1 & 26,7 & 84,8 \\
\hline 40 & 10 & & 55,9 & 31,7 & 87,6 \\
\hline Totale & 52 & & 59,1 & 28,1 & 87,2 \\
\hline
\end{tabular}


Di tutt'altra specie, invece, il panorama che ci si prospetta se guardiamo al voto di lista: qui, la crisi dei grandi partiti di massa e il quadro composito delle due coalizioni maggiori si riflette apertamente nella caduta dell'indice di bipolarismo (FIG. 23) che accompagna l'intero arco del periodo qui considerato: se a metà degli anni Ottanta, i due partiti maggiori assommavano mediamente circa i tre quarti dei voti, questa quota è progressivamente diminuita, sino a toccare, nelle elezioni del 2000-2004, un valore del 53,4\%. Va rilevato, peraltro, come la caduta in termini percentuali (confrontando il 1983-1985 con il 2000-2004), riguardi soprattutto il primo partito $(-14,1 \%)$ e in misura inferiore il secondo $(-7,2 \%)$ : un dato che non sorprende più di tanto, se consideriamo le vicende politiche che hanno vissuto il PCI e i partiti che ne hanno ereditato, in Toscana, la cospicua dote elettorale. In termini relativi, tuttavia, si mantengono le distanze, per così dire: con la seconda lista che ottiene sempre, all'incirca, la metà dei voti della prima ${ }^{27}$.

FIG. 23 - Toscana. Indice di bipolarismo. Percentuale media di voto alle prime due liste. 51 comuni con oltre 15 mila abitanti.

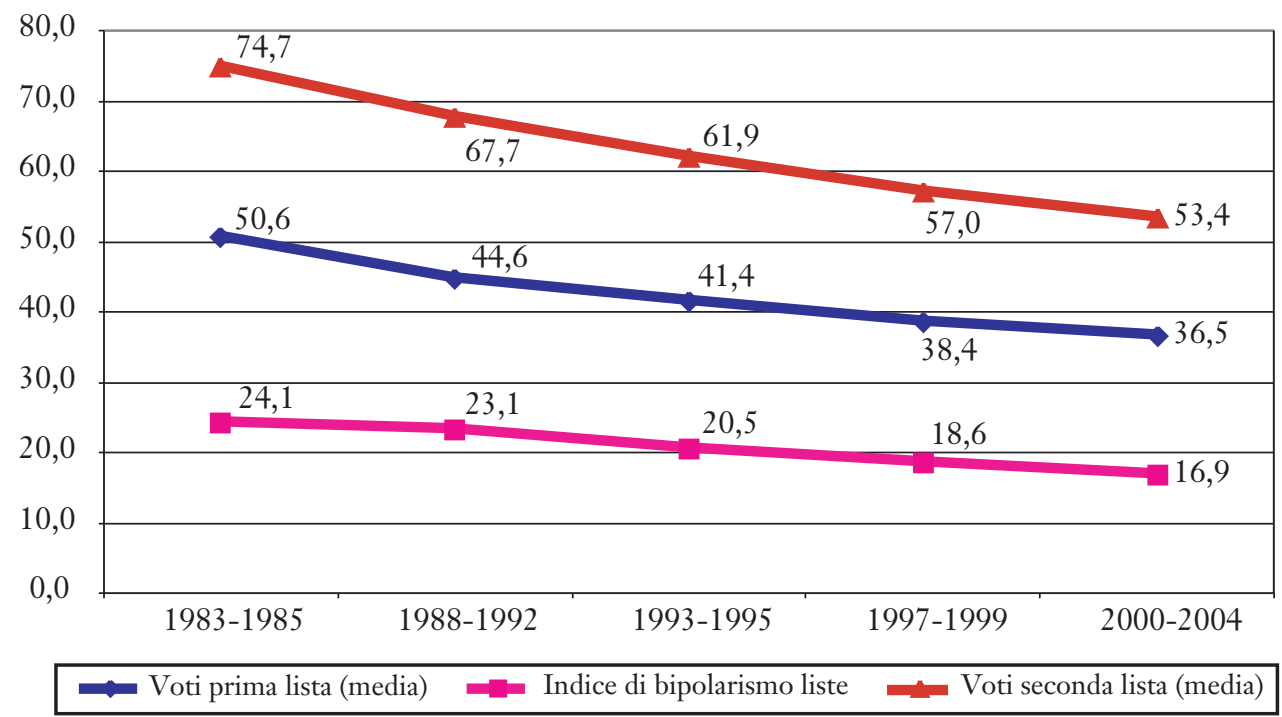

Siamo dunque lontani dai valori dei decenni precedenti: le prime due liste si collocano oggi intorno o poco sopra la metà dei voti: ad un'elevata concentrazione del voto ai primi due candidati si abbina una notevole frammentazione nel voto ai partiti. Resta tuttavia ancora da rispondere ad un altro interrogativo, già sopra richiamato: al di là del peso dei primi due candidati e delle prime due liste, quale è il formato dei sistemi politici locali, così come emerge dalla vicenda del voto ammi-

27 Precisiamo che l'indice di bipolarismo qui considerato riguarda la media delle percentuali riportate nei comuni oggetto della nostra indagine, senza tener conto del loro diverso peso demografico: e questo, perché ci interessa capire, in questa sede, la struttura della competizione nei comuni, e non tanto il comportamento degli elettori nel loro complesso. 
nistrativo degli ultimi vent'anni, prima e dopo la riforma del 1993? Quale, cioè, il peso effettivo e relativo delle varie forze in campo?

$\mathrm{Ci}$ può aiutare a cercare una risposta a questi interrogativi l'analisi di quella particolare misura che viene definita «numero effettivo» dei partiti (un indice che, per brevità, da qui in avanti, definiremo anche come indice Neff), secondo una formula oramai consolidata in letteratura, e che, nel nostro caso, può essere applicata, oltre che ai partiti elettorali (sulla base dei voti) e ai partiti consiliari (sulla base dei seggi), anche ai candidati ${ }^{28}$.

I tasselli dell'analisi fin qui condotta trovano conferma anche da questa misurazione (FIG. 24): mentre il numero effettivo dei partiti, nell'arco di vent'anni, è cresciuto in maniera costante e lineare, passando da un indice Neff elettorale che denotava un sistema sostanzialmente tripolare (3.0) ad un indice di 5,1 (ossia, 5 partiti rilevanti), e da un indice Neff consiliare di 2,7 ad un indice di 4,6; l'andamento dell'indice relativo ai candidati conferma invece una struttura della competizione progressivamente sempre più bipolare $(2,3$ nell'ultimo ciclo elettorale), con un residuo che non giunge a configurarsi come un'alternativa consistente. Inoltre, come emerge dai dati relativi anche alle diverse classi dimensionali dei comuni (per i dati completi, si veda la TAB. 14), si conferma una prevedibile correlazione tra la diversa ampiezza dei consigli e il grado di concentrazione e/o frammentazione del sistema dei partiti e delle coalizioni (quali si esprimono attraverso il numero di candidati).

Per valutare i mutamenti indotti dal nuovo sistema elettorale, abbiamo fin qui ripercorso tutti gli aspetti legati all'offerta elettorale e quelli legati alla concentrazione e/o alla frammentazione dei sistemi politici locali. Abbiamo cercato cioè di comprendere se, al di là del numero di liste concorrenti e/o rappresentate, i sistemi politici locali abbiano vissuto una peculiare evoluzione nel senso di un incremento del numero di partiti effettivamente rilevanti, tanto per ciò che riguarda il voto degli elettori (il voto è più disperso o più concentrato, rispetto al passato?), quanto per ciò che concerne le stesse assemblee elettive (la rappresentanza è più, o meno, frammentata?). Le risposte che abbiamo ottenuto vanno tutte nella direzione più volte indicata: quella di sistemi politici locali che certamente tendono a strutturarsi in forma bipolare (per quanto non senza residui e tensioni), attorno al ruolo dei candidati sindaci e alle coalizioni che li sostengono; ma che, nello stesso tempo, per gli stessi incentivi sistemici che le regole elettorali contengono (oltre che per i caratteri complessivi che il nostro sistema politico ha via via assunto, nel corso della transizione degli anni Novanta), tende a riprodurre un elevato grado di frammentazione della rappresentanza, attraverso l'incremento del numero di liste presentate agli elettori e attraverso un elevato numero di liste che riescono ad accedere ai consigli

${ }^{28}$ Si tratta della formula di Laakso-Taagepera, (originariamente proposta nel saggio«"Effective" Number of Parties: a Measure with Applications to West Europe», in Comparative Political Studies, , 1979, 1, pp. 3-27). Si vedano anche A. LijPHART, Electoral Systems and Party Sistems, Oxford, Oxford University Press, 1994, pp.67 e sgg.; e il volume di G. BALDINI e A. PAPPALARDO, Sistemi elettorali e partiti nelle democrazie contemporanee, Roma-Bari, Laterza, 2004 (sul «numero effettivo», pp. 12-14). 
comunali (nonostante la forte riduzione dei seggi previsti dalle nuove norme). A questo punto, si tratta di comprendere come si inserisca, in questo quadro, la nuova figura dei sindaci e come interpretare quello che sembra uno dei tratti dominanti della nuova forma del governo locale, la personalizzazione della competizione.

FIG. 24 - Toscana. Numero effettivo di partiti e candidati. Comuni con oltre 15 mila abitanti.

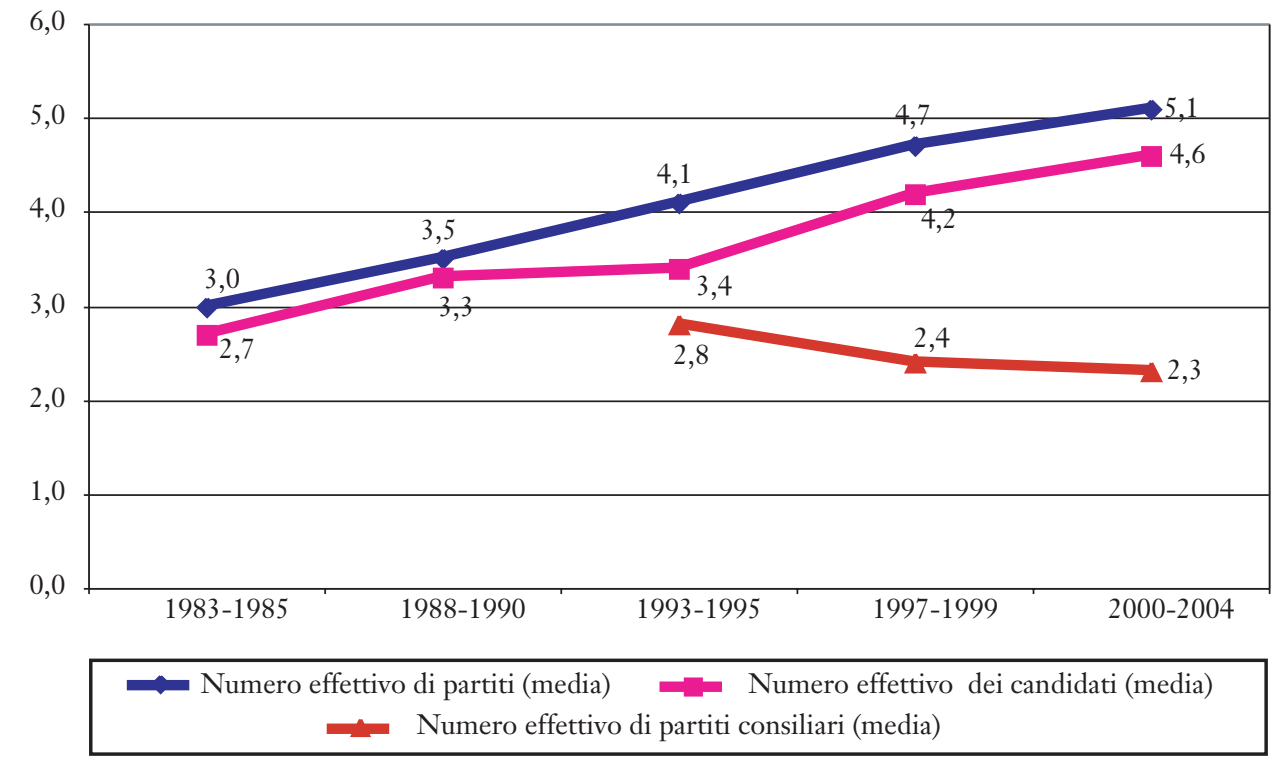

TAB. 14 - Toscana. Media dei candidati, media delle liste e numero effettivo di candidati e liste. Comuni con oltre 15 mila abitanti.

\begin{tabular}{cccccccc}
\hline $\begin{array}{c}\text { Dimensione del } \\
\text { Consiglio comu- } \\
\text { nale (n. seggi) }\end{array}$ & $\begin{array}{c}\text { N. di } \\
\text { comuni }\end{array}$ & $\begin{array}{c}\text { Ciclo } \\
\text { elettorale }\end{array}$ & $\begin{array}{c}\text { Candidati } \\
\text { sindaco } \\
\text { (media) }\end{array}$ & $\begin{array}{c}\text { Liste } \\
\text { (media) }\end{array}$ & $\begin{array}{c}\text { N. eff. } \\
\text { candidati }\end{array}$ & $\begin{array}{c}\text { N. effettivo } \\
\text { partiti (voti) }\end{array}$ & $\begin{array}{c}\text { N. effettivo } \\
\text { partiti } \\
\text { (seggi) }\end{array}$ \\
\hline 20 & 30 & $1993-1995$ & & & & & \\
30 & 11 & & 4,0 & 5,7 & 2,7 & 3,7 & 3,0 \\
$>40$ & 10 & & 4,8 & 7,6 & 2,6 & 4,3 & 3,7 \\
Totale & 51 & & 6,5 & 11,1 & 3,2 & 5,4 & 4,1 \\
& & 4,6 & 7,2 & 2,8 & 4,1 & 3,4 \\
20 & 30 & & & & & & \\
30 & 11 & & 4,1 & 7,7 & 2,4 & 4,2 & 3,6 \\
$>40$ & 10 & & 6,0 & 10,1 & 2,1 & 5,2 & 4,8 \\
Totale & 51 & & 4,6 & 9,4 & 2,5 & 5,9 & 5,6 \\
& & & & & & 4,4 & 4,2 \\
20 & 30 & & 3,5 & 9,1 & 2,2 & 4,6 & 4,1 \\
30 & 12 & & 4,1 & 12,2 & 2,3 & 5,7 & 5,0 \\
$>40$ & 10 & & 6,4 & 16,5 & 2,4 & 6,1 & 5,8 \\
\hline Totale & 52 & & 4,2 & 11,2 & 2,3 & 5,1 & 4,6 \\
\hline
\end{tabular}




\section{Sindaci e personalizzazione}

Le misure del «valore aggiunto» dei candidati. - Nel primo paragrafo, dedicato alla partecipazione elettorale, ci siamo già soffermati sul fenomeno del voto personalizzato, osservato soprattutto in relazione alle diverse modalità di voto che la nuova legge elettorale consente e quindi guardando in particolare al rapporto tra $\mathrm{i}$ voti espressi ai soli candidati sindaco e il totale degli elettori (o il totale di voti validi). Secondo questa ottica, il voto personalizzato appare come una nuova modalità di partecipazione, un'opportunità ulteriore di scelta offerta agli elettori. Attraverso la possibilità di un voto esclusivo ai candidati sindaci, inoltre, la sfera della competizione tra i candidati (e tra le coalizioni) acquista una maggiore autonomia, si svincola dal voto di partito e dalla competizione tra i partiti.

Si tratta ora di misurare e valutare il fenomeno del voto personalizzato da un altro punto di vista, ossia guardare a come esso incida sulla dinamica e sulla logica della competizione, quale sia il ruolo dei candidati sindaci e della loro diversa figura (per coalizione e per posizione, come sindaci uscenti o come sfidanti, ecc.) e quali siano le variabili esplicative che incidono sulle dimensioni e la qualità del fenomeno.

Vi sono stati, nel corso degli anni, diverse ipotesi di misurazione del voto personalizzato; ma, al di là dei diversi indici utilizzabili, la sostanza del problema non muta: si tratta di calcolare quello che comunemente viene oramai definito il «valore aggiunto» dei candidati alla carica di sindaco, ossia l'entità del voto esclusivamente espresso ai candidati sindaco, rispetto ai voti validi espressi alle liste collegate. Tale differenza si può esprimere semplicemente in termini percentuali, o attraverso numeri indice (facendo base 100 il totale dei voti di lista), o ancora attraverso un particolare indice che sottrae il valore 1 al rapporto tra i voti del candidato e i voti delle liste che lo sostengono ${ }^{29}$. Nelle pagine seguenti, analizzeremo tale fenomeno dapprima per l'insieme dei candidati, poi sulla base della loro posizione nella competizione, poi sulla base della loro appartenenza alle diverse coalizioni e, infine, ci soffermeremo su un particolare tipo di candidati, i sindaci uscenti, ricandidati e (in Toscana, quasi sempre) rieletti. Utilizzeremo, intanto, la versione più semplice dell'indice di personalizzazione (d'ora in poi abbreviando in IP), quella cioè calcolata sulla base della differenza percentuale.

29 Questa versione dell'indice di personalizzazione è stata proposta da G. BALDINI e G. LEGNANTE, Città al voto, cit., pp. 211-212, e permette di fissare una scala in cui al valore 0 corrisponde il caso di una perfetta identità tra voti al candidato e voti alle liste che lo sostengono e al valore 1 il caso di un candidato che raccolga esattamente il doppio del voto alle liste. Vi è dunque un valore minimo fisso di -1 , e un valore superiore che invece, in linea teorica, come scrivono i due autori, «si approssima all'infinito positivo quanto maggiori sono i voti del candidato e quanto minori quelli delle liste che lo appoggiano». Questa versione dell'indice di personalizzazione, come vedremo in seguito, permette di essere meglio comparata ad un altro indice, quello di attrazione, che si calcola sulla stessa scala numerica. 
Abbiamo visto in precedenza le dimensioni generali del fenomeno (FIG. $9 \mathrm{e}$ ТАВ. 6): negli ultimi due cicli elettorali, circa il $9 \%$ dei voti validi sono espressi ai soli candidati. Per valutarne meglio i caratteri, tuttavia, non basta considerare il livello complessivo di personalizzazione: occorre guardare anche in che misura i diversi candidati concorrano a produrre un tale effetto. E i candidati, a loro volta, possono essere distinti per la posizione che occupano nella competizione e per lo schieramento politico cui appartengono ${ }^{30}$.

Misure e variabili di rendimento: voto personale e posizione del candidato. - Cominciamo ad osservare la posizione dei candidati, ossia in che misura le dimensioni del voto personalizzato investano i diversi candidati secondo il loro piazzamento nella competizione (al primo turno). I dati mostrano un risultato che, sulle prime, può risultare sorprendente (FIG. 25 e TAB. 15): non sembrano sussistere, infatti, variazioni significative di rendimento tra i candidati che si collocano al primo posto, quelli che si piazzano al secondo posto e anche tutti gli altri successivi candidati. Anzi, in alcuni casi, e per alcuni cicli elettorali, il rendimento del secondo e del terzo candidato e quello degli altri, appare superiore a quello dei candidati che si piazzano al primo posto (e che, in gran parte, risulteranno poi eletti: tranne i pochi casi in cui, al ballottaggio, si produce un ribaltamento delle posizioni ${ }^{31}$ ). Questi dati ci invitano alla cautela interpretativa e sollecitano un interrogativo di fondo: è giusto misurare il successo di un candidato solo mediante il cosiddetto valore aggiunto? E quali sono i fattori che determinano veramente l'indice di personalizzazione di un candidato e l'entità dello scarto tra voti personali e voti di lista?

\footnotetext{
${ }^{30}$ Non sono ancora disponibili ricerche e studi relativi all'insieme dei comuni italiani chiamati al voto per la terza volta tra il 2000 e il 2004. Sul fenomeno del voto personale ai candidati sindaci, si veda la nota curata per l'Istituto Cattaneo da P. CORBETTA e D. TUORTO, La personalizzazione del voto per il sindaco (comunicato del 15 giugno 2004), relativa però solo ai 30 comuni capoluoghi di provincia impegnati nelle elezioni del 2004. Gli autori rilevano nel complesso un voto personalizzato pari al 7,6\% dei voti validi. In particolare, nei 13 capoluoghi della "zona rossa", tale percentuale è pari al 7,5\% (10,6\% nei capoluoghi del Nord; 6,3\% in quelli del Sud).

${ }^{31}$ In Toscana, i casi in cui un candidato, giunto secondo al primo turno, risulta poi vincente al ballottaggio, sono pochi: in particolare, solo 3 su 19 nel 1993-1995 (Camaiore, Massarosa e Montecatini Terme: in tutti i tre casi è il candidato del centrosinistra a vincere il ballottaggio); un solo caso (su 8) nel 1997-1999 (Arezzo: qui è il candidato del centrodestra vincente al ballottaggio a ribaltare le posizioni). Con l'ultimo ciclo elettorale 2000-2004, la frequenza dei ribaltamenti è maggiore, con ben 5 casi su un totale di 10 ballottaggi: a Camaiore, Pescia (2001) e ancora ad Arezzo, a vantaggio del candidato del centrodestra, e a Carrara e Capannori, a vantaggio del candidato del centrosinistra.
} 
FIG. 25 - Toscana. Indice di personalizzazione, per posizione dei candidati. Comuni con oltre 15 mila abitanti.

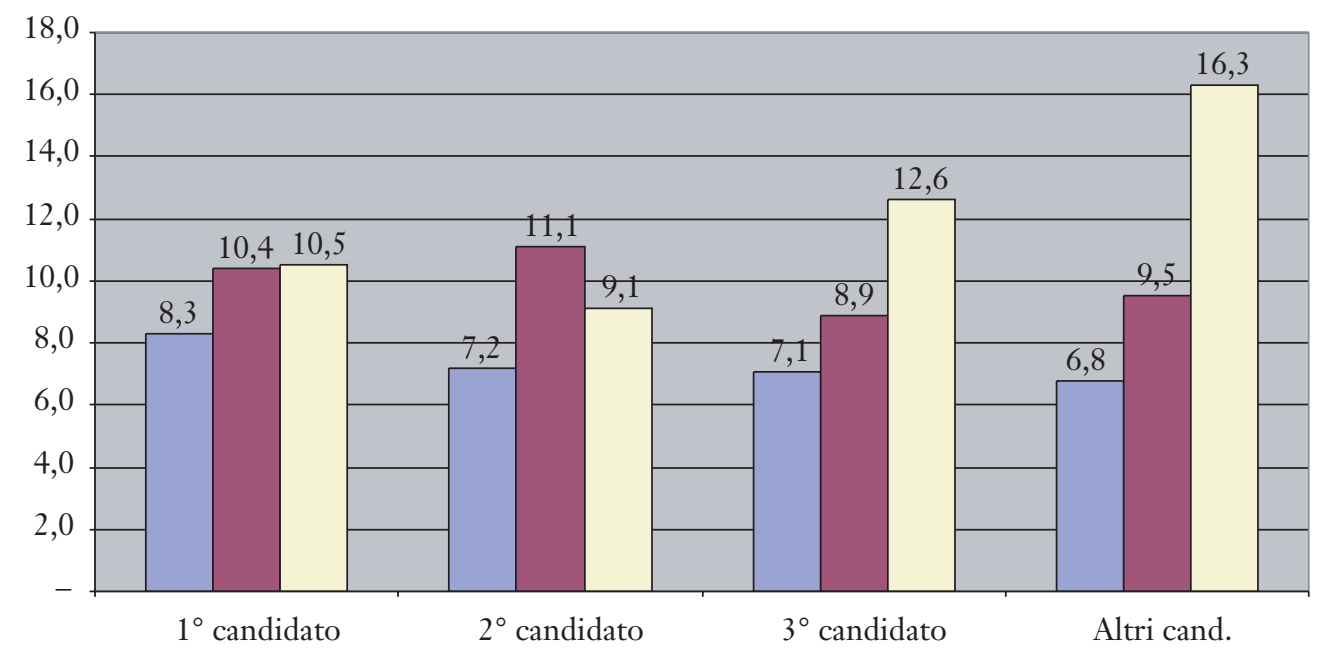

$$
\square \text { 1993-95 } \square \text { 1997-99 } \square \text { 2000-04 }
$$

TAB. 15 - Toscana. Indici di personalizzazione, per posizione dei candidati. Differenza percentuale media tra voti personalizzati ai candidati e voti di lista.

\begin{tabular}{lrrrrrrrr}
\hline \multirow{2}{*}{$\begin{array}{c}\text { Ampiezza } \\
\text { Consigli }\end{array}$} & \multicolumn{1}{c}{$1^{\circ}$ candidato } & \multicolumn{2}{c}{$2^{\circ}$ candidato } & \multicolumn{2}{c}{$3^{\circ}$ candidato } & \multicolumn{2}{c}{ Altri candidati } \\
\cline { 2 - 9 } & Media & Totale & \multicolumn{1}{c}{ Media } & Totale & Media & Totale & Media & Totale \\
\hline \multirow{2}{*}{ 20 seggi } & 7,7 & 6,8 & 4,7 & 5,0 & 6,4 & 6,7 & 7,4 & 6,7 \\
30 seggi & 10,4 & 8,8 & 8,9 & 9,2 & 6,8 & 8,4 & 4,5 & 4,6 \\
$>$ 40 seggi & 7,9 & 7,1 & 11,9 & 9,8 & 9,3 & 8,6 & 7,8 & 5,8 \\
Totale & 8,3 & 7,4 & 7,0 & 8,5 & 7,1 & 8,1 & 6,8 & 5,8 \\
\hline \multicolumn{7}{c}{$1997-1999(51$ comuni) } \\
\hline 20 seggi & 9,9 & 9,6 & 9,7 & 9,9 & 7,8 & 7,7 & 7,6 & 7,0 \\
30 seggi & 12,5 & 10,6 & 14,3 & 14,4 & 9,3 & 10,4 & 10,4 & 9,7 \\
$>40$ seggi & 9,9 & 8,7 & 11,9 & 11,0 & 11,8 & 9,9 & 10,9 & 9,5 \\
Totale & 10,4 & 9,4 & 11,1 & 11,3 & 8,9 & 9,3 & 9,5 & 8,9 \\
\hline \multicolumn{7}{c}{$2000-2004(52$ comuni) } \\
\hline 20 seggi & 9,7 & 8,7 & 8,6 & 8,4 & 10,6 & 8,4 & 10,1 & 7,8 \\
30 seggi & 12,6 & 10,3 & 8,8 & 9,7 & 21,2 & 20,1 & 8,6 & 7,1 \\
$>40$ seggi & 10,3 & 8,2 & 10,8 & 9,0 & 8,4 & 13,4 & 22,4 & 22,7 \\
Totale & 10,5 & 8,8 & 9,1 & 9,0 & 12,6 & 13,5 & 16,3 & 16,1 \\
\hline
\end{tabular}


In effetti, a ben guardare, non sono poche le insidie metodologiche che si nascondono dietro questa misura, a cui oramai si ricorre frequentemente, anche nei commenti politici e giornalistici post-elettorali ${ }^{32}$. Non è solo la capacità attrattiva personale o la popolarità di un candidato, ad entrare in gioco, ma una serie di altre variabili, per molti versi decisive: in primo luogo, il numero delle liste a sostegno dei candidati. Abbiamo già visto in precedenza (vedi sopra le FIGG. 1516-17) quanto fosse stato rilevante, nel determinare un progressivo incremento delle liste concorrenti e la frammentazione dell'offerta, il ruolo dei candidati espressi dalle maggiori coalizioni: possiamo ora approfondire questo elemento guardando in particolare alla media delle liste presentate a sostegno dei candidati giunti rispettivamente primi e secondi (FIG. 26): le liste a sostegno del candidato primo sono state mediamente 2,6 nel 1993-1995, 4,3 nel 1997-1999 e 5,2 nel 2000-2004; quelle a sostegno del candidato secondo, rispettivamente 1,6, e poi 2,4 e 3,2. Perché questa variabile è decisiva? Per il semplice motivo che entra qui in gioco il meccanismo, più volte ricordato, del "trasferimento" automatico del voto dalle liste ai candidati: è chiaro che, quante più liste concorrono a sostenere un candidato, tanto maggiore è la possibilità che una quota di elettori voti anche per una lista, (magari in funzione del voto di preferenza per scegliere un consigliere). Possiamo dire dunque che il rendimento di un candidato, se misurato solo attraverso la quota di voti personali rispetto al totale dei voti delle liste a sostegno, rischia di rivelarsi una misura fallace della qualità della candidatura stessa: possono intervenire, infatti, vari ordini di fattori che deprimono l'indice di personalizzazione, ossia:

a) in generale, il grado di strutturazione dei partiti: un sistema politico locale che veda partiti forti e radicati nel territorio, in grado di produrre una buona mobilitazione nella campagna elettorale o in grado di presentare candidati di buona qualità alla carica di consigliere, tende ad attirare e sollecitare una maggiore quantità di voti anche sulle liste, quale che sia il grado di attrazione e di popolarità del candidato sindaco. Può anche accadere così che un candidato forte e popolare non veda riflessa questa sua forza d'attrazione in uno scarto elevato tra voti personali e voti di lista, in quanto anche i partiti mostrano un'elevata capacità di mobilitazione e di raccolta di consenso sulle proprie liste e sui propri candidati alla carica di consigliere. Oppure, al contrario, può darsi che un candidato relativamente meno forte registri uno scarto positivo molto elevato, proprio per la debolezza delle liste che lo sostengono;

b) in particolare, il numero delle liste a sostegno di una candidatura può giocare un ruolo determinante nell'abbassare l'indice di personalizzazione: la presenza di

\footnotetext{
${ }^{32}$ Nei commenti post-elettorali, spesso, si incorre in un altro, ancor più grave, errore di "lettura" dei risultati, laddove si confrontano semplicemente le percentuali di voto al candidato e la somma delle percentuali del voto alle liste: si tratta, evidentemente, di percentuali calcolate su valori assoluti diversi e quindi incomparabili. In molti casi, un candidato che ottiene una percentuale inferiore a quella delle proprie liste, registra comunque un rendimento positivo, in quanto il numero dei voti ottenuti è più alto di quello delle liste.
} 
un numero elevato di liste, allargando l'offerta elettorale, aumenta la probabilità che venga espresso anche un voto di lista, con ciò stesso riducendo lo scarto tra voti personali e voti di lista;

c) di converso, candidati minori (o, caso piuttosto frequente, candidati che sono espressione di uno o più partiti riconducibili ad uno dei due maggiori schieramenti, che "sono in rotta" con il resto della coalizione), alla ricerca di maggiore visibilità, e che corrono da soli al fine di misurare il livello del proprio consenso personale, possono con maggiore probabilità registrare scarti positivi rispetto ai voti delle liste di sostegno.

FIG. 26 - Toscana. Media delle liste a sostegno del sindaco eletto e del $2^{\circ}$ candidato. Comuni con oltre 15 mila abitanti.

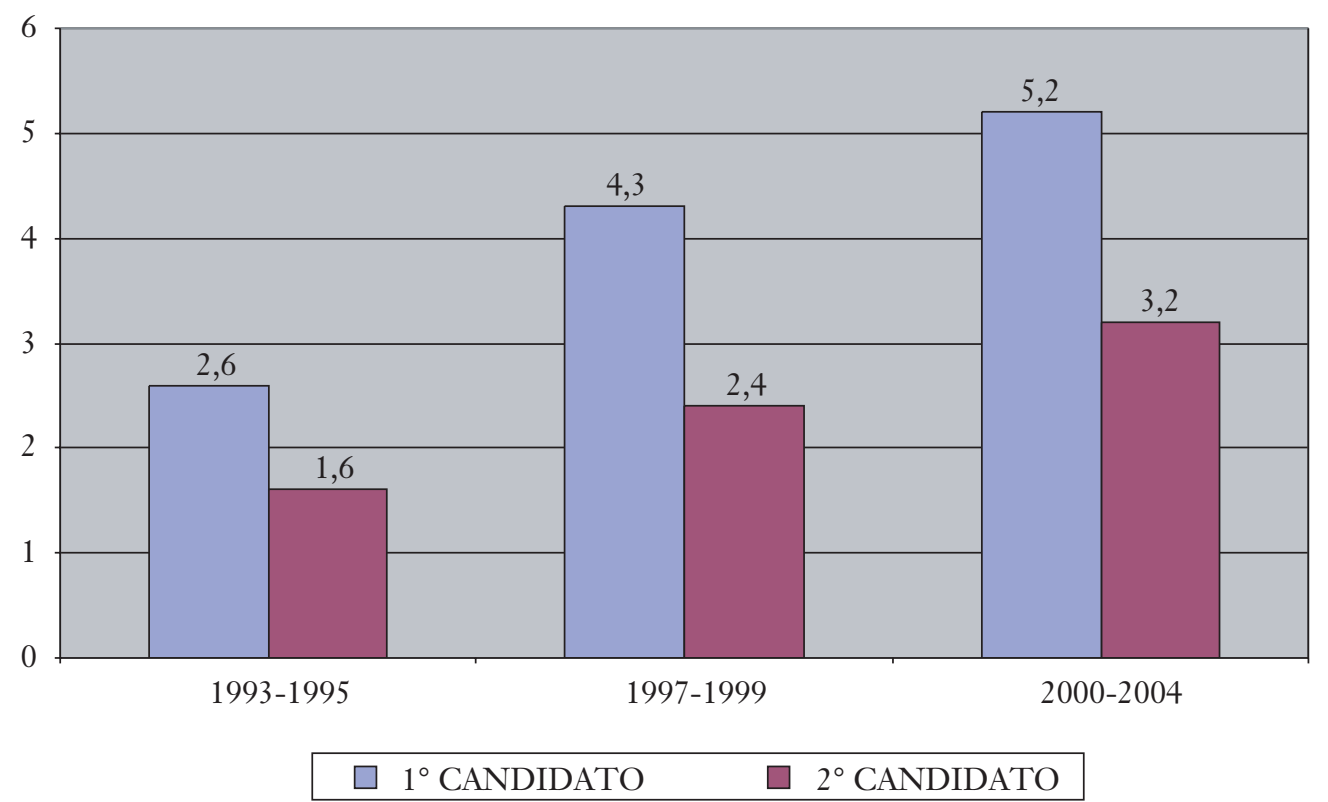

Misure e variabili di rendimento: voto personale e coalizioni di sostegno. - Pur con queste avvertenze critiche, l'indice di personalizzazione rimane una misura della dinamica competitiva a cui è impossibile rinunciare: la cautela interpretativa, a cui devono indurre le considerazioni appena svolte, spinge piuttosto ad affiancare la lettura di questo indice ad altre possibili misure del rendimento dei candidati e ad un'analisi differenziata della figura stessa dei candidati.

È possibile infatti analizzare l'indice di personalizzazione dei candidati anche sulla base della loro appartenenza ad uno schieramento e al tipo di coalizione che li sostiene (FIG. 27 e TAB. 16). 
FIG. 27 - Toscana. Indice di personalizzazione dei candidati, per schieramento politico. Differenza percentuale media tra voti personalizzati ai candidati e voti di lista. Comuni con oltre 15 mila abitanti.

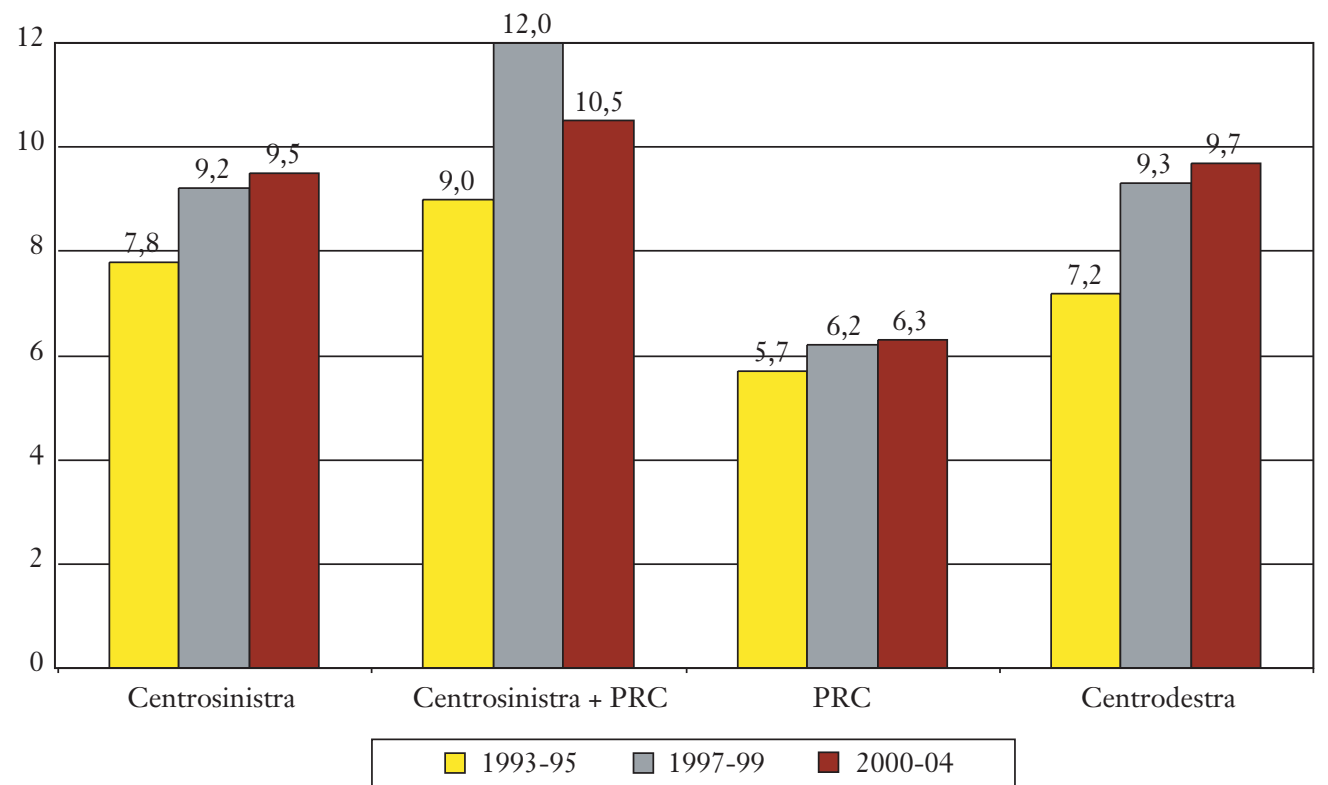

TAB. 16 - Toscana. Indice di personalizzazione dei candidati, per schieramento politico. Differenza percentuale media tra voti personalizzati ai candidati e voti di lista. Comuni con oltre 15 mila abitanti.

\begin{tabular}{lccc}
\hline 51 Comuni & \multicolumn{3}{c}{$1993-1995$} \\
\hline & n. candidati & Media & Totale \\
\cline { 2 - 4 } Centrosinistra & 28 & 7,8 & 5,9 \\
Centrosinistra + PRC & 21 & 9,0 & 9,0 \\
PRC e Prc+altri & 30 & 5,7 & 6,7 \\
Centrodestra & 46 & 7,2 & 8,8 \\
\hline & & & \\
\hline 51 Comuni & n. candidati & Media & Totale \\
\hline & 36 & 9,2 & 8,5 \\
Centrosinistra & 13 & 12,0 & 11,2 \\
Centrosinistra + PRC & 37 & 6,2 & 6,0 \\
PRC e Prc+altri & 51 & 9,3 & 11,2 \\
Centrodestra & & & \\
\hline & & $2000-2004$ & Motale \\
\hline 52 Comuni & n. candidati & 9,5 & 7,8 \\
\hline & 31 & 10,5 & 8,7 \\
Centrosinistra & 23 & 6,3 & 11,3 \\
Centrosinistra + PRC & 29 & 9,7 & 10,2 \\
PRC e Prc+altri & 51 & & \\
Centrodestra & &
\end{tabular}


Dall'analisi dei dati, oltre alla conferma di una crescita, tra i due cicli elettorali, del voto personale a tutti i candidati, emerge come tale crescita, per le due coalizioni maggiori, abbia riguardato in particolare i candidati del centrodestra, che da una media dell'IP del 7,4\% passano, nel ciclo elettorale successivo, ad una media del 10,9\% (+2,5\%, superiore a quella dei candidati sostenuti dal centrosinistra e da Rifondazione, $+2,3 \%$, o dal solo centrosinistra, $+2,1 \%$ ). L'altro dato interessante da rilevare riguarda il diverso rendimento dei candidati sostenuti dal solo centrosinistra e quello dei candidati sostenuti anche da Rifondazione Comunista: questi ultimi toccano, nelle elezioni del 1997-1999, un IP dell' $11,3 \%$, a fronte del 9,5\% dei primi. Da una parte, quindi, sembra messa in dubbio un'immagine piuttosto diffusa, secondo cui il centrodestra soffrirebbe, a livello locale, di una certa carenza di candidati forti e credibili: non si direbbe, stando a questi indici. E d'altra parte, come vedremo in seguito, gli stessi esiti delle consultazioni (specie a partire dal 1997-1999) mostrano la capacità del centrodestra di proporre candidature che si sono rivelate vincenti. E tuttavia, forse valgono qui proprio quelle avvertenze critiche sopra richiamate: è possibile cioè che incida anche il livello di strutturazione e di radicamento dei partiti, la capacità di mobilitazione che essi sono in grado di esprimere e la stessa capacità di attivazione dei candidati consiglieri. Quindi, per il centrodestra, si può ipotizzare la presentazione, in genere, di buoni candidati, il cui rendimento positivo può risultare però accentuato dalla relativa debolezza dei partiti che li sostengono e dal minor numero di liste che compongono la coalizione. Dall'altra parte, per lo schieramento di centrosinistra, può valere il fenomeno opposto: buoni candidati (o anche ottimi, se guardiamo al fatto che, poi, nella maggior parte dei casi, alla fin fine, sono loro a vincere), ma il cui rendimento può risultare relativamente più basso in quanto hanno alle proprie spalle, per un verso, una coalizione più ampia e variegata, con numerose liste: e, per un altro verso, partiti sicuramente più strutturati, (in particolare, ovviamente, i DS, ma anche gli eredi del voto democristiano), con un tradizionale insediamento organizzativo nel territorio che, per quanto logorato, garantisce comunque un più elevato grado di visibilità e una maggiore capacità di raccolta di consenso.

In questo quadro, merita particolare attenzione il dato relativo ai candidati del centrosinistra che hanno alle spalle l'intera coalizione e, in più, anche il sostegno di Rifondazione Comunista: in questi casi, il rendimento personale dei candidati appare particolarmente elevato. Una possibile spiegazione rimanda forse alla figura di questi candidati: proprio perché espressione di una coalizione ampia, è possibile che essi tendano a caratterizzarsi in maniera più autonoma, a distaccare cioè maggiormente il loro profilo politico, rispetto all'insieme dei partiti che li appoggiano, e quindi ad accentuare $i$ tratti di personalizzazione della competizione. E che il rendimento di un candidato sia strettamente legato al tipo e al grado di consenso dei partiti collegati, viene dimostrato a contrario anche dal dato relativo ai candidati sindaco che sono espressi dalla sola Rifondazione Comunista, quando questo partito partecipa isolatamente alla gara eletto- 
rale: in questo caso, l'IP è piuttosto basso, a segnalare come qui prevalga il voto alla lista di partito e un tipo di consenso che è innanzi tutto espressivo di un'identità politico-culturale. Valgono qui anche le dinamiche coalizionali: quando Rifondazione corre da sola, con un proprio candidato sindaco, ciò accade evidentemente per marcare una distinzione dal centrosinistra (quando i rapporti locali tra i partiti, ad esempio, hanno portato ad una rottura di precedenti alleanze) ed è del tutto conseguente che, in questi casi, sia soprattutto il voto di lista ad esprimere gli orientamenti di questa fetta dell'elettorato, con una minore attenzione prestata alla figura del candidato sindaco (a cui si attribuiscono scarse possibilità di successo) ${ }^{33}$.

L'alto indice di personalizzazione dei candidati altri, o anche quello dei candidati riconducibili ad una particolare componente delle coalizioni di centrodestra o di centrosinistra (quando concorrono autonomamente) ci confermano, ancora una volta, la necessità di una particolare cautela interpretativa: si tratta di candidati che, come sappiamo dalle analisi svolte in precedenza, riescono a catturare una fetta modesta di consensi, quella quota cioè di elettori che non si riconoscono nei candidati delle due maggiori coalizioni: eppure, come vediamo, sono candidati che ottengono un rendimento elevato, rispetto ai consensi che conseguono le liste di supporto. Anche in questo caso, una possibile spiegazione va ricercata nella logica della competizione e nel rapporto candidati-liste: i candidati terzi sono candidati che ritengono di dover partecipare alla competizione pensando di poter incamerare un consenso personale, appunto, mentre minore rilievo assume la lista di sostegno (molto spesso una sola). In particolare, possiamo ipotizzare che si tratti a) di candidati riconducibili comunque ad uno dei due schieramenti, che rompono con la propria coalizione, cercando di verificare (ed eventualmente, contrattare) il proprio livello personale di consensi ${ }^{34}$; o $b$ ) candidati di liste locali non coalizzate, che godono di una relativa notorietà in ambito locale, espressione di peculiari issues locali (sono stati abbastanza frequenti i casi, in Toscana, di liste civiche costruite attorno a comitati locali, e ai loro leader, su particolari temi: ad esempio, la dismissione di un ospedale o la localizzazione di un inceneritore dei rifiuti).

$33 \mathrm{Vi}$ è un solo caso in cui un candidato di Rifondazione riesce a giungere al ballottaggio, a Borgo San Lorenzo (1995). In altri tre casi, il candidato di Rifondazione giunge secondo, ma il candidato del centrosinistra vince già al primo turno (Certaldo 1999, Castelfiorentino 1995 e ancora Borgo San Lorenzo nel 2004).

34 Abbastanza frequente il caso di candidati (in taluni casi, ex-sindaci) che, non riuscendo ad ottenere l'investitura da parte della propria coalizione, cercano una sorta di "rivalsa", proponendo una propria autonoma candidatura. 
Il rendimento di un candidato: l'indice di attrazione. - Abbiamo visto dunque come le misure di rendimento dei candidato, se effettuate solo attraverso lo scarto tra voti personali e voti di lista, presentino alcuni rischi interpretativi, che è possibile evitare considerando altre variabili esplicative: il numero delle liste a sostegno e il grado di strutturazione dei partiti.

Al fine di ottenere un'immagine più realistica della dinamica competitiva, e delle stesse performances dei vari candidati, appare perciò opportuno combinare l'IP (calcolato secondo l'altra formula sopra indicata) ${ }^{35}$ con un altro indice, che viene definito di attrazione (IA), attraverso cui si misura la capacità di un candidato di attrarre una quota più o meno ampia del voto degli elettori che esprimono un voto personalizzato o un voto diviso ${ }^{36}$. Combinando i due indici, abbiamo così un duplice confronto: il rendimento di ciascun candidato, da una parte, viene comparato a quello delle liste che lo sostengono e, dall'altra parte, a quello degli altri candidati alla carica di sindaco.

L'indice di attrazione, così concepito, riflette più direttamente il livello dei consensi di un candidato e delle proprie liste: candidati dal rendimento positivo, ma dai consensi modesti, possono essere così opportunamente distinti dai candidati che, magari hanno un rendimento personale più basso, ma a partire da livelli decisamente più alti di consenso.

Inoltre, l'indice di attrazione permette anche di considerare, nella misura del possibile, le dimensioni del voto diviso, calcolando il numero minimo di elettori che hanno espresso un tale tipo di voto (il solo misurabile, peraltro, sulla base dei dati aggregati, che non possono cogliere eventuali flussi incrociati tra

35 Si veda, sopra, la nota 30.

36 Per una definizione dell'indice di attrazione, si veda G. BALDINI e G. LEGNANTE, Città al voto, cit., pp. 210-218: l'IA è «dato dal rapporto fra: la differenza fra i voti ottenuti da ciascun candidato sindaco e la somma di quelli ottenuti dalle liste che lo appoggiano, al numeratore, e la somma dei voti personalizzati e dei voti divisi espressi in un comune, al denominatore. In questo modo si ottiene un indice tanto più elevato quanto maggiore è lo scarto nei voti fra il candidato sindaco e le liste che lo appoggiano e quanto minore è la quota di voto personalizzato "disponibile" nel comune (calcolabile, naturalmente, solo ex-post)» (p. 212). E importante rilevare che lo scarto tra voti personali e voti di lista, per ciascun candidato, va rapportato non al totale aggregato dei voti personalizzati, ma alla somma di questi ultimi e dei voti divisi: vanno considerati cioè anche i casi di rendimento "negativo", in quanto tra i voti personalizzati "disponibili", in questo caso, rientrano anche i voti di quegli elettori che, pur votando una lista, hanno votato per un altro candidato. Riportiamo un esempio: nel comune di Campi Bisenzio, elezioni 1995, la differenza tra il totale dei voti ai candidati sindaco e il totale dei voti alle liste è stata di 778 voti; tuttavia, 4 dei 6 candidati hanno riportato meno voti delle proprie liste; l'indice di attrazione va dunque calcolato rapportando, per ciascun candidato, la differenza tra voti personali e voti di lista alla somma dei voti personali e dei voti divisi, ossia nel nostro esempio a 949 (somma della differenza positiva di due candidati: + 907 e +42 ; gli altri quattro candidati assommavano un saldo negativo di 171 voti; per cui $949-171=778$ voti, che era appunto la differenza aggregata tra voti ai sindaci e voti alle liste). In questo caso, il candidato che ha conseguito 907 voti personalizzati (su 949) ottiene un indice di attrazione elevatissimo, pari al $95 \%$ circa dei voti personalizzati "disponibili”. 
voti divisi e voti personalizzati): in Toscana, comunque, il fenomeno appare piuttosto marginale ${ }^{37}$.

La considerazione congiunta sia della personalizzazione che dell'attrazione permette una visione più realistica della dinamica competitiva che si è creata in un comune, distinguendo diversi modelli di competizione: da quella fortemente personalizzata e monopolizzata (in cui un solo candidato abbina valori elevati sia per l'IP che per l'IA), a quella più equilibrata (in cui i vari candidati mostrano valori simili di personalizzazione, ma valori diversi di attrazione: in questo caso, pesa il diverso livello di consenso dei candidati e dei partiti); dalle competizioni in cui è basso (o addirittura negativo) l'IP di molti candidati ed elevato l'IA di uno solo tra di essi; e così via ${ }^{38}$.

Se collochiamo su un piano i due indici, con l'indice di personalizzazione sull'asse delle ordinate e quello di attrazione sull'asse delle ascisse, otteniamo una rappresentazione efficace dei modelli di competizione che si sono configurati nelle elezioni amministrative che stiamo analizzando (FIGG. 28-29-30 e TAB. 17) ${ }^{39}$.

Nel 1993-1995 (vedi la FIG. 28), emerge chiaramente una struttura competitiva in cui ciò che soprattutto incide è la diversa capacità di attrazione dei candidati, mentre meno netto appare il divario tra gli indici di personalizzazione dei vari candidati: vale a dire, non è il diverso valore aggiunto personale dei candidati a fare la differenza, ma la loro diversa forza politica ed elettorale, che si riflette abbastanza omogeneamente e proporzionalmente nella loro diversa capacità di catturare una quota dei voti personali e divisi disponibili. Si tratta dunque di una lettura che non sottovaluta la dimensione personalizzata della competizione, ma ne circoscrive il senso e ne contestualizza la portata.

37 Il fenomeno del voto diviso (altrove in Italia piuttosto rilevante, specie in Sicilia), in Toscana non sembra molto diffuso: i candidati che riportano meno voti delle proprie liste, nell'arco dei tre cicli elettorali, sono stati solo 36 su 687 , cioè il 5,2\% del totale dei candidati. Inoltre, ben 26 casi su 36 sono concentrati nel solo primo ciclo elettorale, e, per la maggior parte di essi, si tratta di uno scarto negativo di pochissime unità. È significativo anche notare come, nella maggior parte dei casi (10) si tratti proprio di candidati espressi da Rifondazione Comunista. Completano il quadro: 5 candidati di area DC/PPI; 8 candidati espressi da singoli partiti dell'area di centrodestra; 7 candidati di liste locali, 2 del PSI, e un candidato ciascuno per la lista Pannella, il PRI, lo SDI e i Verdi. Nel complesso, i voti divisi "visibili" (i soli che possono emergere dall'analisi dei dati aggregati) sono appena 4.555, su un totale di voti espressi (a 687 candidati) di 4.484.395: cioè lo 0,1\%). Se rapportato agli elettori la percentuale di voto diviso è pari allo $0,07 \%$, cioè un valore ancora più basso di quello riscontrato da Baldini e Legnante (p. 150) per le regioni centrali italiane, 0,2\%, e notevolmente inferiore al 2,4\% delle regioni meridionali e soprattutto al 5,5\% della Sicilia.

${ }^{38}$ Per l'illustrazione dei diversi modelli di competizione e dei risultati delle analisi condotte sull'insieme dei comuni italiani, per grandi aree geografiche, si veda G. BALDINI e G. LEGNANTE, Città al voto, cit., pp.219-223.

39 Anche in questo caso si tratta di medie, e non di indici calcolati sul totale dei voti validi: va tuttavia ancora notato come una verifica in questo senso non abbia prodotto risultati dissimili, a significare una certa omogeneità dei fenomeni nei diversi comuni, quale che sia la loro dimensione demografica. 
FIG. 28 - Toscana. Indici di personalizzazione e di attrazione, per posizione dei candidati (media di 51 comuni con oltre 15mila abitanti - 1993-1995).

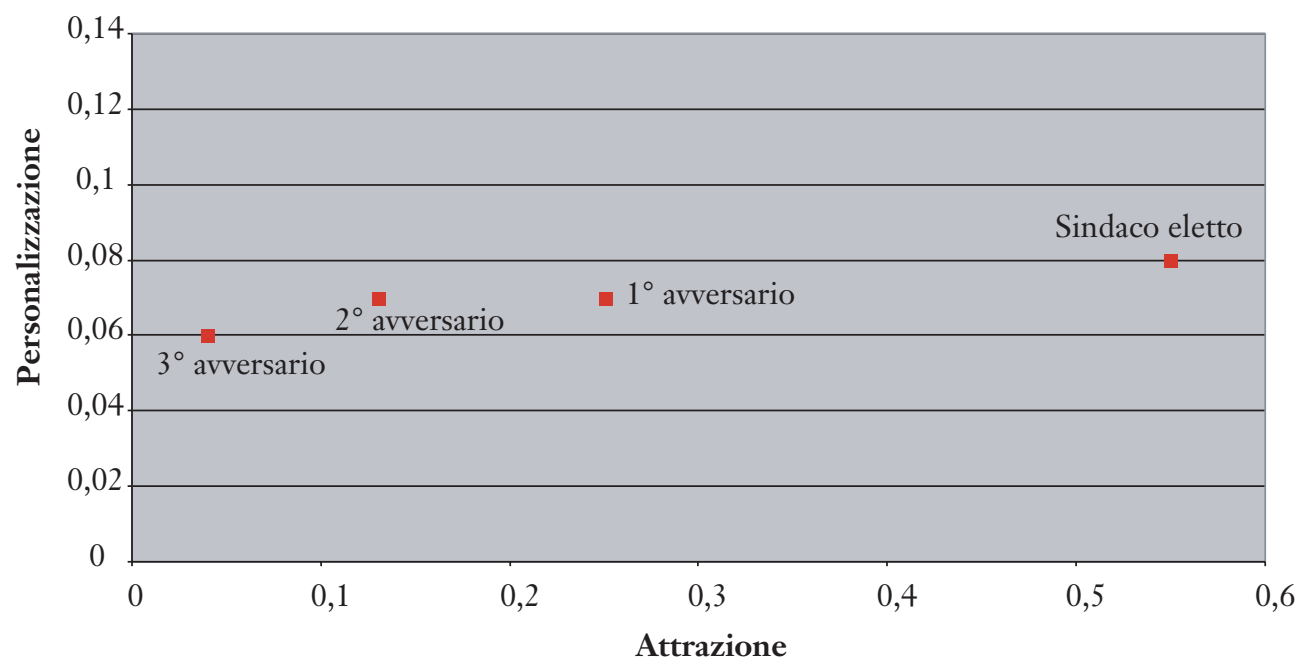

TAB. 17 - Toscana. Indici di personalizzazione (IP) e di attrazione (IA), per posizione dei candidati (media di 51 comuni con oltre 15 mila abitanti).

\begin{tabular}{lcccccccccc}
\hline & \multicolumn{3}{c}{$1993-1995$} & \multicolumn{3}{c}{$1997-1999$} & \multicolumn{3}{c}{$2000-2004$} \\
\cline { 2 - 10 } & N. casi & IP & IA & N. casi & IP & IA & N. casi & IP & IA \\
\hline Sindaci eletti & 51 & 0,08 & 0,55 & 51 & 0,11 & 0,58 & 52 & 0,10 & 0,58 \\
$1^{\circ}$ avversario & 51 & 0,07 & 0,25 & 51 & 0,11 & 0,29 & 52 & 0,10 & 0,27 \\
$2^{\circ}$ avversario & 50 & 0,07 & 0,13 & 49 & 0,09 & 0,09 & 46 & 0,13 & 0,10 \\
$3^{\circ}$ avversario & 36 & 0,06 & 0,04 & 37 & 0,08 & 0,04 & 27 & 0,10 & 0,05 \\
\hline
\end{tabular}

I dati relativi al ciclo elettorale 1997-1999 (vedi la FIG. 29) mostrano invece una maggiore apertura competitiva del confronto elettorale: il confronto si fa cioè un po' più serrato, e si può cogliere questa tendenza nel maggiore incremento dei due indici relativi al primo sfidante del sindaco risultato poi eletto, rispetto alla crescita di questi stessi indici che pure viene registrata anche dai candidati vincenti (e lo sfidante, ricordiamo, in 6 casi è ora appartenente al centrosinistra; mentre, nel primo ciclo, i primi avversari erano tutti di centrodestra). Rispetto al modello competitivo precedente emerge (anche visivamente, confrontando i due grafici) una più elevata differenziazione dell'IP, che ora appare molto più elevato per $\mathrm{i}$ primi due candidati; ma, ancor più, risalta uno stacco netto tra l'IA dei primi due contendenti e quello degli altri due candidati: ovvero, la dinamica bipolare si fa più intensa. 
FIG. 29 - Toscana. Indici di personalizzazione e di attrazione, per posizione dei candidati (media di 51 comuni con oltre 15mila abitanti - 1997-1999).

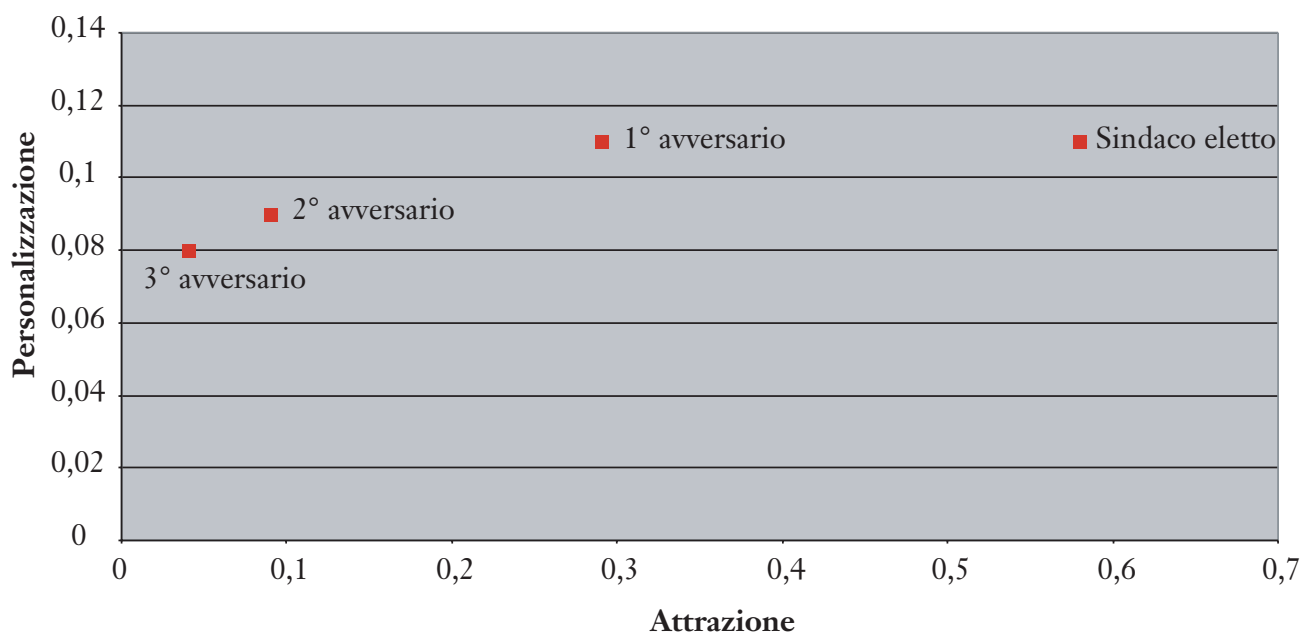

I dati relativi al terzo ciclo elettorale 2000-2004 (FIG. 30) mostrano una stabilità degli indici di attrazione dei sindaci eletti e di tutti gli altri contendenti; l'indice di personalizzazione, invece, si conferma come un dato che, assunto isolatamente, rischia di risultare fuorviante: per quest'ultima serie di dati, ad esempio, gli IP dei secondi e dei terzi avversari risultano addirittura in crescita.

FIG. 30 - Toscana. Indici di personalizzazione e di attrazione, per posizione dei candidati (media di 51 comuni con oltre 15mila abitanti - 2000-2004).

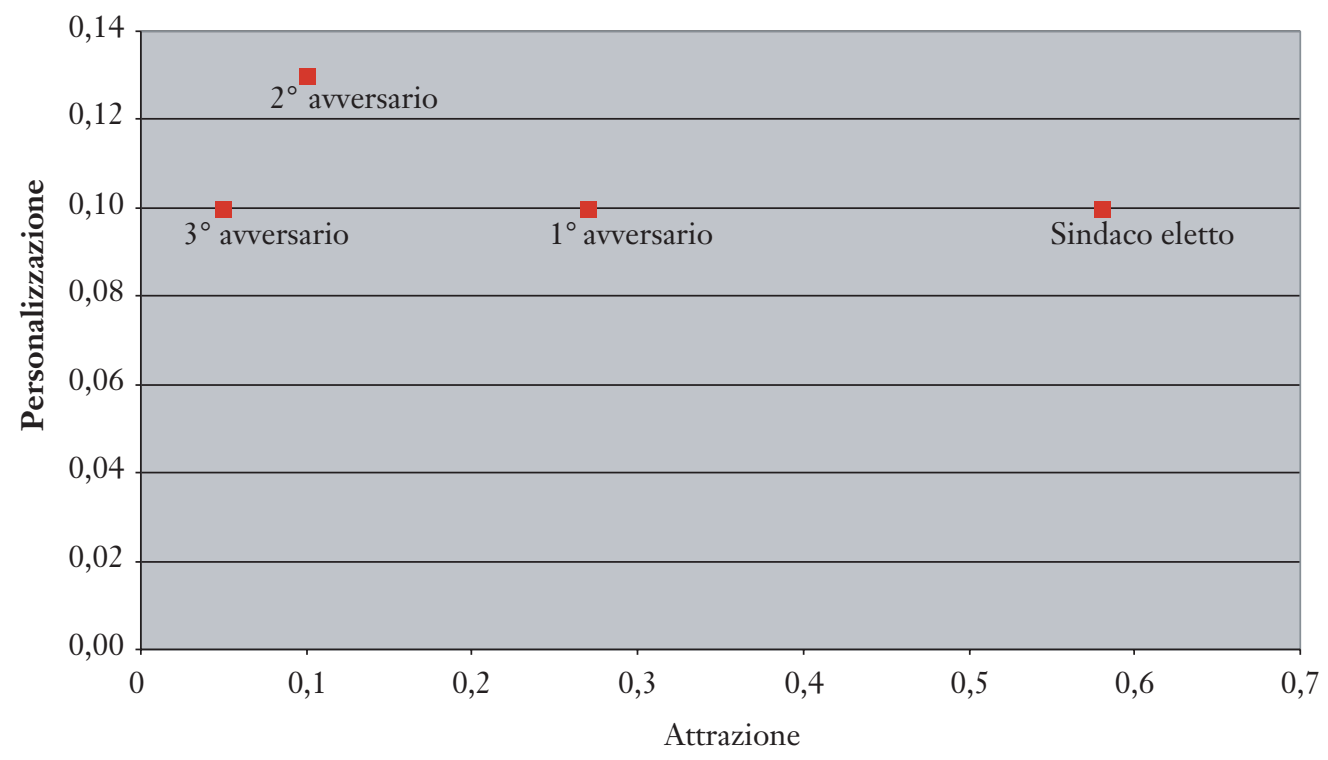


Personalizzazione e attrazione possono essere misurate non solo, come abbiamo fatto finora, rispetto alla posizione dei candidati quale emerge dalla competizione, ma anche in relazione allo schieramento che li sostiene. Anche in questo caso, l'immagine un po' appiattita che emergeva dalla sola analisi dei voti personali raccolti da un candidato, viene meglio messa a fuoco dalla contemporanea considerazione del grado di attrazione che un candidato esercita (FIGG. 31-32-32 е ТАВ. 18).

FIG. 31 - Toscana. Indici di personalizzazione e di attrazione, per schieramento dei candidati. Comuni con oltre 15 mila abitanti (1993-1995).

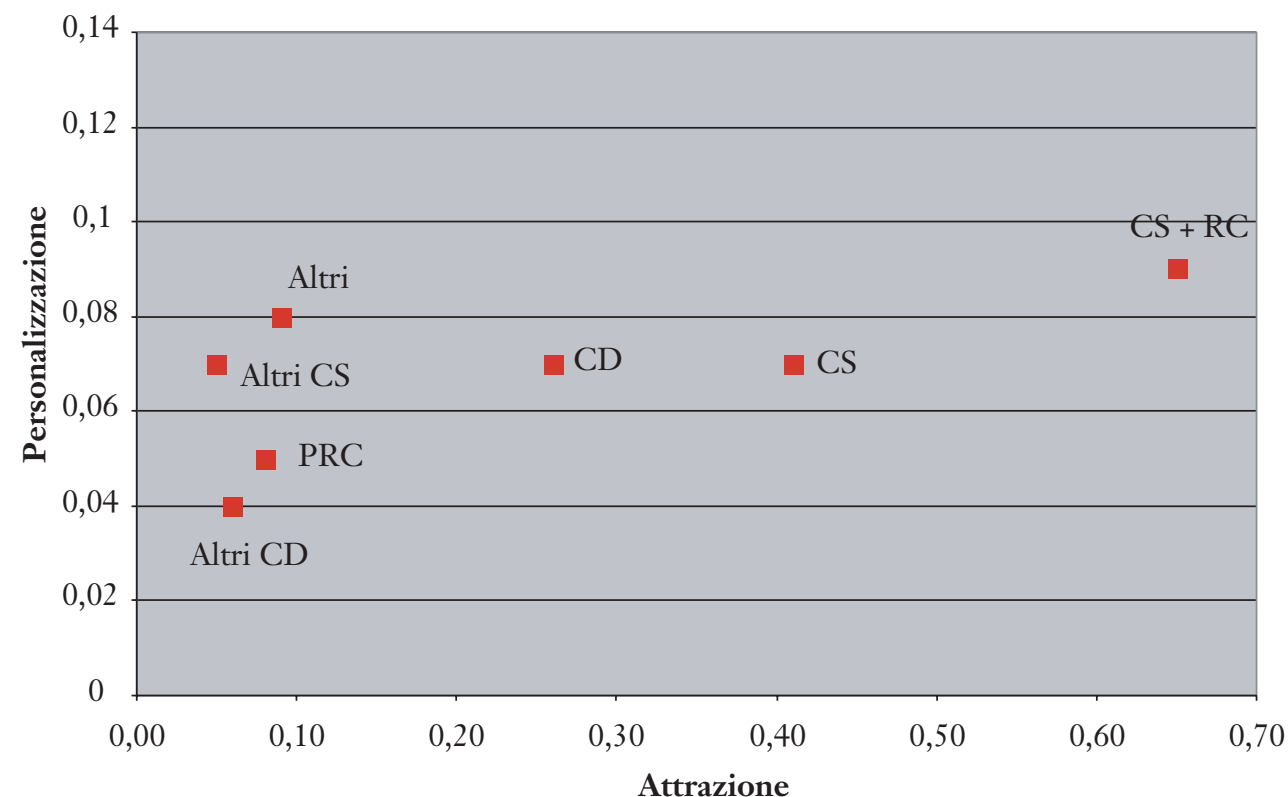

Se guardiamo al primo ciclo elettorale (FIG. 31), i candidati sorretti unitariamente dall'intero centrosinistra e da Rifondazione (e, come abbiamo visto, spesso da liste unitarie) registrano ottime performances da entrambi i punti di vista (IP $=0,09$, ossia in termini percentuali, circa il $9 \%$ di voti in più rispetto alle liste; $\mathrm{IA}=0,65$, ossia i $2 / 3$ del totale dei voti personali e divisi espressi); i candidati del centrodestra e del centrosinistra (senza RC) hanno un indice di personalizzazione simile, ma capacità diverse di attrazione; tutti gli altri (compresi i candidati di Rifondazione Comunista, quando hanno alle spalle solo la lista di partito o, in qualche caso, un'altra lista locale o talvolta $\mathrm{i}$ Verdi) hanno indici di personalizzazione variabili, (talvolta anche elevati, come nel caso soprattutto dei candidati altri, ossia soprattutto candidati di liste civiche non coalizzate), ma indici di attrazione molto modesti. 
Il quadro cambia sostanzialmente con il secondo ciclo elettorale (FIG. 32): i candidati del centrodestra e quelli unitari di centrosinistra e Rifondazione hanno un indice di personalizzazione leggermente più elevato rispetto a quello dei candidati del solo centrosinistra (che pure crescono da 0,07 a 0,10 ), ma questi ultimi si staccano nettamente per un più elevato grado di attrazione (che cresce da 0,41 a 0,57$)$. Rispetto al ciclo elettorale precedente, sembrano perdere forza le candidature unitarie CS + RC, mentre i candidati del centrodestra migliorano in modo notevole, soprattutto dal versante della personalizzazione (da 0,07 a 0,11), limitandosi però a riflettere invece, quanto ad "attrazione", il peso elettorale della coalizione (circa un terzo degli elettori). Per il resto, crescono notevolmente gli IP di tutti gli altri candidati (con l'eccezione dei soli candidati di $\mathrm{RC}$, che passano appena da 0,05 a 0,06 ), ma rimane modesta la loro capacità di attrazione.

FIG. 32 - Toscana. Indici di personalizzazione e di attrazione, per schieramento dei candidati. Comuni con oltre 15 mila abitanti (1997-1999).

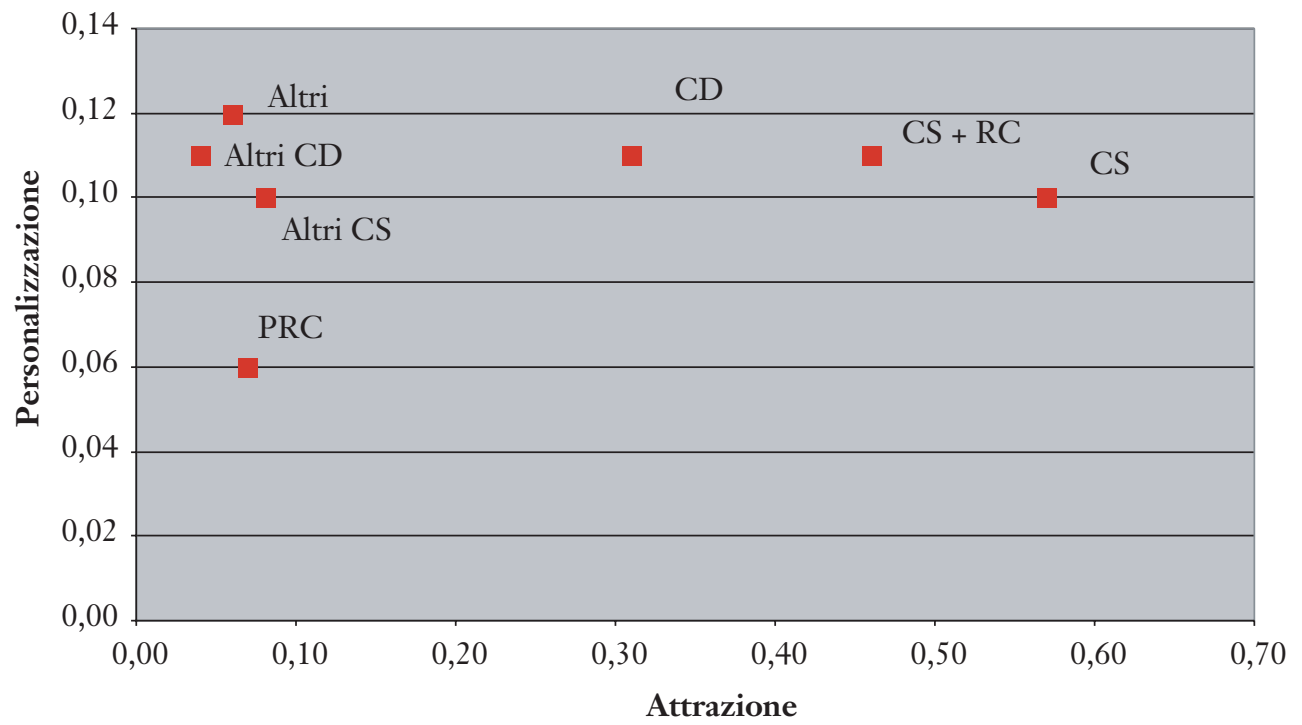

Il terzo ciclo elettorale, infine, (FIG. 33), mostra una sostanziale stabilità dei vari indici, se si eccettua una notevole ripresa dei candidati "unitari" del centrosinistra e di RC, che tornano ad un livello molto elevato di "attrazione" $(\mathrm{IA}=0,59)$. 
FIG. 33 - Toscana. Indici di personalizzazione e di attrazione, per schieramento dei candidati. Comuni con oltre 15 mila abitanti (2000-2004).

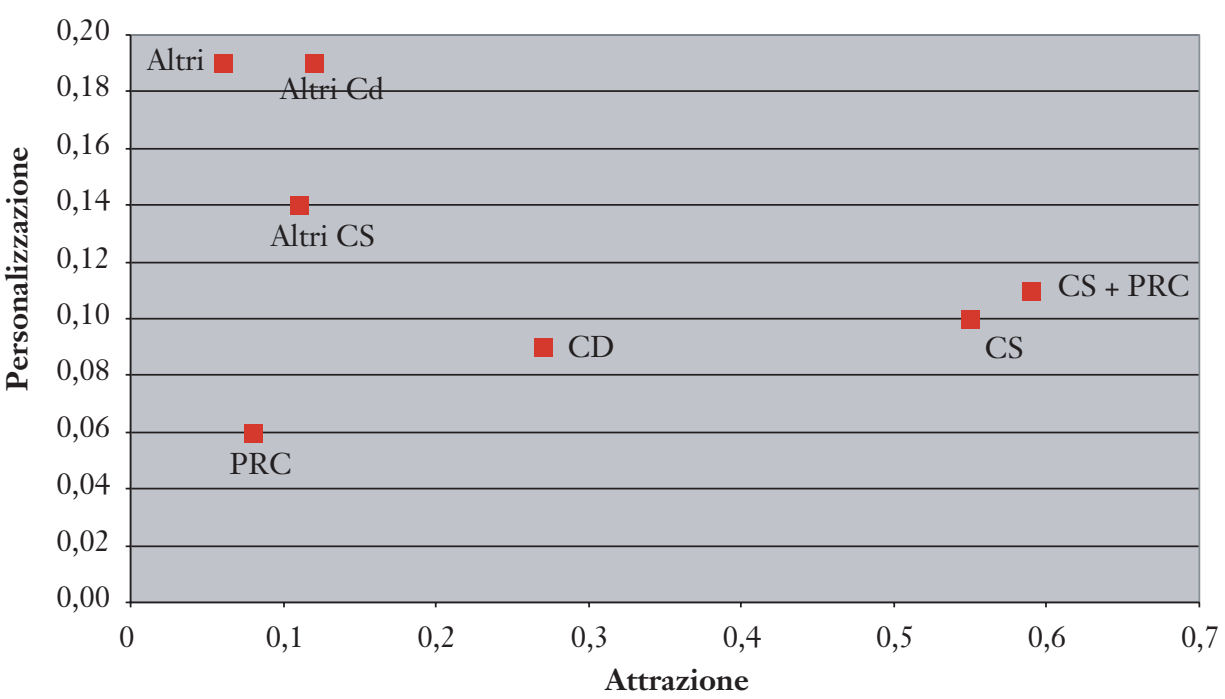

TAB. 18 - Toscana. Indici di personalizzazione (IP) e di attrazione (IA), per schieramento dei candidati. Comuni con oltre 15 mila abitanti.

\begin{tabular}{|c|c|c|c|}
\hline \multirow[t]{2}{*}{51 Comuni } & \multicolumn{3}{|c|}{$1993-1995$} \\
\hline & n. candidati & IA & IP \\
\hline Centrosinistra & 30 & 0,41 & 0,07 \\
\hline Centrosinistra + PRC & 21 & 0,65 & 0,09 \\
\hline PRC & 29 & 0,08 & 0,05 \\
\hline Centrodestra & 45 & 0,26 & 0,07 \\
\hline Altri Centrosinistra & 13 & 0,05 & 0,07 \\
\hline Altri Centrodestra & 29 & 0,06 & 0,04 \\
\hline Altri & 69 & 0,09 & 0,08 \\
\hline Totale & 236 & & \\
\hline \multirow[t]{2}{*}{51 Comuni } & \multicolumn{3}{|c|}{ 1997-1999 } \\
\hline & n. candidati & $\overline{\mathrm{IA}}$ & IP \\
\hline Centrosinistra & 37 & 0,57 & 0,10 \\
\hline Centrosinistra + PRC & 14 & 0,46 & 0,11 \\
\hline PRC & 37 & 0,07 & 0,06 \\
\hline Centrodestra & 50 & 0,31 & 0,11 \\
\hline Altri Centrosinistra & 20 & 0,08 & 0,10 \\
\hline Altri Centrodestra & 37 & 0,04 & 0,11 \\
\hline Altri & 37 & 0,06 & 0,12 \\
\hline Totale & 232 & & \\
\hline \multirow[t]{2}{*}{52 Comuni } & \multicolumn{3}{|c|}{$2000-2004$} \\
\hline & n. candidati & IA & IP \\
\hline Centrosinistra & 30 & 0,55 & 0,10 \\
\hline Centrosinistra + PRC & 22 & 0,59 & 0,11 \\
\hline PRC & 29 & 0,08 & 0,06 \\
\hline Centrodestra & 51 & 0,27 & 0,09 \\
\hline Altri Centrosinistra & 12 & 0,11 & 0,14 \\
\hline Altri Centrodestra & 10 & 0,12 & 0,19 \\
\hline Altri & 61 & 0,06 & 0,19 \\
\hline
\end{tabular}


Il rendimento dei sindaci uscenti e ricandidati. - Appare infine significativo misurare i livelli di personalizzazione e di attrazione di una particolare "categoria" di candidati: i sindaci incumbents, ossia i sindaci eletti e ricandidati nei vari cicli elettorali, a partire dal 1993-1995.

Nel primo ciclo elettorale tutti i 51 comuni toscani con oltre 15 mila abitanti videro l'elezione di un sindaco di centrosinistra, ovviamente con una notevole varietà nella composizione delle coalizioni di sostegno. Quattro anni dopo, nelle elezioni svoltesi tra il 1997 e il 1999 (ТАВ. 19), i sindaci ricandidati e rieletti sono stati 29, mentre sono stati 16 i casi di avvicendamento (conferma del precedente schieramento, ma con un altro candidato e, spesso, con una diversa composizione della coalizione $)^{40}$ e 6 i casi di alternanza, ossia i casi in cui è avvenuto un cambio di maggioranza (in tutti e sei casi, dal centrosinistra al centrodestra: in un solo caso battendo il sindaco uscente). Nel terzo ciclo elettorale il quadro è più mosso: i sindaci del centrosinistra ricandidati e rieletti sono 14, quelli del centrodestra 5 (su sei uscenti: uno solo verrà sconfitto, e si tratta peraltro dell'unico caso di sindaco incumbent che non verrà riconfermato ${ }^{41}$.Vi sono poi, nel complesso, ben 29 casi di avvicendamento: va notato peraltro come si tratta quasi sempre (26 casi) di un "avvicendamento forzato", in quanto i sindaci uscenti di centrosinistra avevano già completato il proprio secondo mandato ${ }^{42}$. In questa terza tornata elettorale il centrosinistra, dunque, ha mostrato di tenere conto maggiormente dei rischi legati alla mancata ricandidatura di un sindaco uscente, facendo tesoro in questo senso di un insegnamento oramai piuttosto diffuso e di cui in anche in Toscana si era potuto fare diretta esperienza, ad esempio nel caso delle elezioni di Lucca del 1998.

\footnotetext{
${ }^{40}$ Per completezza di informazione, va anche ricordato come non tutti i casi di avvicendamento, cioè di mancata ricandidatura del sindaco uscente, hanno lo stesso significato: vi sono infatti 4 casi di sindaci "longevi", che pur avendo svolto un solo mandato sulla base della nuova normativa, non si ricandidano avendo alle spalle già un primo mandato, essendo già stati eletti, con le vecchie regole, dopo le elezioni svoltesi nel 1990 (si tratta dei sindaci di Monsummano Terme, Poggibonsi, Pontedera, San Miniato). I sindaci più "longevi” sono comunque quelli di Campi Bisenzio, Quarrata, Siena e Rosignano Marittimo, che pur essendo già stati eletti dopo il voto del 1988-1990, vengono ricandidati e rieletti sia nel 1993-1995 che nel 1997-1999.

41 Si tratta del comune di Capannori: la vicenda si presenta peraltro piuttosto singolare, in quanto il sindaco uscente ha partecipato ugualmente alle elezioni, risultandone sconfitto, pur essendo stato sottoposto, alla vigilia della presentazione delle candidature, ad un provvedimento giudiziario di arresti domiciliari.

${ }^{42}$ I casi in cui il sindaco uscente non è stato ricandidato sono quelli di Carrara e Cortona. Nel caso di Bagno a Ripoli vi è stato il decesso del sindaco eletto nel 1999.
} 
TAB. 19 - Sindaci in Toscana: conferme, avvicendamenti e alternanze. Comuni con oltre 15 mila abitanti.

\begin{tabular}{|c|c|c|c|}
\hline & $1993-1195$ & $\begin{array}{r}1993-1995 / \\
1997-1999\end{array}$ & $\begin{array}{l}1997-1999 \\
2000-2004\end{array}$ \\
\hline Sindaci eletti CS & 51 & 45 & 44 \\
\hline Sindaci eletti CD & 0 & 6 & 8 \\
\hline Riconferme sindaci CS & & 29 & 13 \\
\hline Riconferme sindaci CD & & & 5 \\
\hline Avvicendamenti sindaci CS & & 16 & 6 \\
\hline \multicolumn{4}{|l|}{ Avvicendamenti sindaci CD } \\
\hline $\begin{array}{l}\text { Avvicendamenti sindaci CS, per conclusione } \\
\text { secondo mandato }\end{array}$ & & & 22 \\
\hline \multicolumn{4}{|l|}{$\begin{array}{l}\text { Avvicendamenti sindaci } \mathrm{CD} \text {, per conclusione } \\
\text { secondo mandato }\end{array}$} \\
\hline Alternanze da CS a CD & & 6 & 3 \\
\hline di cui con sindaco CS uscente ricandidato & & 1 & \\
\hline Alternanze da CD CS & & & 2 \\
\hline di cui con sindaco $C D$ uscente ricandidato & & & 1 \\
\hline TOTALE & 51 & 51 & 52 \\
\hline
\end{tabular}

Nota: il Comune di Pescia ha votato nel 2001 e, dopo lo scioglimento del Consiglio, anche nel 2004: nel 2001 vi fu alternanza dal CS al CD, nel 2004 dal CD al CS.

Generalmente, si ritiene che questo altissimo livello di riconferma dei sindaci incumbents sia da attribuire agli indubbi vantaggi che la permanenza in carica offre, rispetto alle chances di un rivale: amministrare un comune per quattro o cinque anni, al di là delle qualità politiche dimostrate nell'azione di governo, consente (entro certi limiti, ovviamente, ma comunque in larga misura) di giovarsi di una popolarità e di una rete di relazioni su cui gli altri concorrenti non possono contare.

Ma come e quanto incide il fattore incumbency? Al di là del rilevante numero di riconferma dei sindaci ricandidati, è possibile misurarne la crescita della popolarità, da un'elezione all'altra? La TAB. 20 cerca di rispondere a queste domande, presentando gli indici IP e IA nei diversi casi di conferma, avvicendamento e alternanza dei sindaci eletti. 
TAB. 20 - Sindaci in Toscana. Conferme, avvicendamenti e alternanze nelle elezioni amministrative. Indici di personalizzazione (IP) e di attrazione (IA). Media. Comuni con oltre 15 mila abitanti. 1993-1995/1997-1999

\begin{tabular}{lrrrr} 
n. casi & \multicolumn{2}{c}{$1993-1995$} & \multicolumn{2}{c}{$1997-1999$} \\
\hline & IP & IA & IP & IA \\
\hline
\end{tabular}

$\begin{array}{llllll}\text { Conferme } & 29 & 0,08 & 0,55 & 0,12 & 0,60\end{array}$

\begin{tabular}{|c|}
\hline Avvicendamenti \\
\hline
\end{tabular}

\begin{tabular}{|c|c|c|c|c|c|c|c|}
\hline & \multirow[t]{3}{*}{ n. casi } & \multirow{2}{*}{\multicolumn{2}{|c|}{$\frac{1993-1995}{\text { sindaco eletto CS }}$}} & \multicolumn{4}{|c|}{ 1997-1999 } \\
\hline & & & & nuovo & lato $\mathrm{CS}$ & sindace & \\
\hline & & IP & IA & IP & IA & IP & IA \\
\hline Alternanze (da CS a CD) & 6 & 0,10 & 0,36 & 0,11 & 0,27 & 0,16 & 0,60 \\
\hline
\end{tabular}

1997-1999/2000-2004

\begin{tabular}{|c|c|c|c|c|c|}
\hline & n. casi & & 99 & & 04 \\
\hline & & IP & IA & IP & IA \\
\hline Conferme CS* & 13 & 0,08 & 0,56 & 0,10 & 0,65 \\
\hline Conferme CD & 5 & 0,17 & 0,61 & 0,16 & 0,60 \\
\hline Avvicendamenti CS & 28 & 0,09 & 0,55 & 0,08 & 0,57 \\
\hline
\end{tabular}

\begin{tabular}{|c|c|c|c|c|c|c|c|}
\hline & \multirow[t]{2}{*}{ n. casi } & \multicolumn{2}{|c|}{ 1997-1999 } & \multicolumn{4}{|c|}{$2000-2004$} \\
\hline & & sindac & tto CD & nuovo & dato $C D$ & sindace & tto CS \\
\hline \multirow{4}{*}{ Alternanze (da CD a CS) } & & IP & IA & IP & $\mathrm{IA}$ & IP & IA \\
\hline & 2 & 0,11 & 0,38 & 0,08 & 0,27 & 0,15 & 0,49 \\
\hline & & \multicolumn{2}{|c|}{ sindaco eletto CS } & \multicolumn{2}{|c|}{ nuovo candidato CS } & \multicolumn{2}{|c|}{ sindaco eletto CD } \\
\hline & & IP & IA & IP & IA & IP & IA \\
\hline Alternanze (da CS a CD) & 3 & 0,23 & 0,61 & 0,23 & 0,5 & 0,14 & 0,36 \\
\hline
\end{tabular}

* I casi di conferma dei sindaci CS sono 13, anziché 14 (come indicato nella tabella precedente), in quanto il comune di Calenzano, nel 1999, aveva votato con il sistema elettorale dei comuni con meno di 15 mila ab.

I dati che presentiamo non sono univoci, e smentiscono, almeno in parte, la linearità delle ipotesi interpretative più diffuse. Guardiamo innanzi tutto al confronto tra il primo e il secondo ciclo elettorale. Il primo elemento che risalta è proprio l'incidenza del fattore incumbency: i sindaci rieletti vedono una crescita tanto dell'IP che dell'IA, passando da una dotazione di voti personali, alla prima elezione, che corrispondeva mediamente ad un incremento dell' $8 \%$ di voti personali, rispetto ai voti di lista, ad uno scarto positivo di 12 punti percentuali, in occasione della rielezione. Anche l'indice di attrazione cresce, sia pure in modo più contenuto (da 0,55 a 
$0,60)$. Una riprova viene dai dati relativi ai comuni in cui è stata confermata una maggioranza di centrosinistra, ma con un nuovo candidato alla carica di sindaco: qui, tanto i valori di IP quanto quelli di IA si abbassano: in modo contenuto i primi, a segnalare probabilmente, ex post, la buona qualità delle nuove candidature; in modo più marcato i secondi, a segnalare probabilmente il migliore rendimento relativo degli altri candidati. Incidono anche, su questi valori, le particolari condizioni che si vengono a creare nei casi in cui non concorra alla rielezione un sindaco uscente, ma tutti gli schieramenti presentino candidati nuovi: infatti, mentre generalmente un sindaco incumbent è un candidato forte (che gode di vantaggi competitivi tali da rendere difficile, talvolta, per lo schieramento avversario, la stessa individuazione di un candidato alternativo altrettanto forte), nei casi in cui si procede invece ad un avvicendamento nella candidatura della maggioranza uscente, è possibile che la competizione appaia più aperta e, conseguentemente, risulti incoraggiata la presenza di candidati alternativi in grado di registrare un buon rendimento. Una tale diversa condizione, possiamo ipotizzare che si rifletta nei diversi andamenti degli indici di attrazione, (più bassi, come abbiamo visto, nei casi di avvicendamento). I casi di alternanza ci confermano, per altro verso, quanto un elevato indice di personalizzazione, se considerato separatamente, non sia di per sé associato sempre al successo di un candidato: i candidati di centrosinistra sconfitti, infatti, mantengono ed anzi accrescono l'IP del loro predecessore (da 0,10 a 0,11 ), ma vedono cadere nettamente l'indice di attrazione (da 0,36 a 0,27 ), a fronte di candidati del centrodestra che vedono, insieme, un più elevato IP ed un altissimo IA $(0,60)$. In questi casi, possiamo concludere, non è stato tanto il cattivo rendimento "personale" dei candidati di centrosinistra a determinare la sconfitta, quanto il migliore rendimento comparato degli avversari, soprattutto in termini di attrazione dei voti personali e divisi disponibili ${ }^{43}$.

Anche da queste considerazioni emerge dunque come sia soprattutto una considerazione congiunta dei due indici a riflettere più compiutamente il contesto di una competizione: laddove non conta solo il rendimento, individuale e relativo, dei candidati, ma evidentemente la forza politica ed elettorale complessiva di una coalizione (ed è bene ricordare che, in generale, sono circa un decimo i voti validi espressi solo per i candidati sindaco: una quota che può rivelarsi decisiva quando la competizione è serrata, ma che in molti altri casi non è sufficiente a rovesciare gli esiti di un voto, quando i rapporti di forza siano più nettamente delineati).

Il confronto tra il primo e il secondo ciclo elettorale sembra confermare dunque le ipotesi che solitamente vengono avanzate nell'analisi del fattore incumbency. L'analisi del terzo ciclo elettorale (si veda ancora la TAB. 20), tuttavia, introduce elementi diversi, che ci costringono ad allargare e complicare il quadro interpretativo. Come abbiamo detto, nelle elezioni svoltesi tra il $2000 \mathrm{e}$

\footnotetext{
${ }^{43}$ Va ricordato peraltro che solo in un caso, sui sei citati di alternanza, il centrosinistra candidava un sindaco uscente, a Grosseto. Negli altri cinque casi di alternanza, il centrosinistra presentava comunque dei nuovi candidati.
}

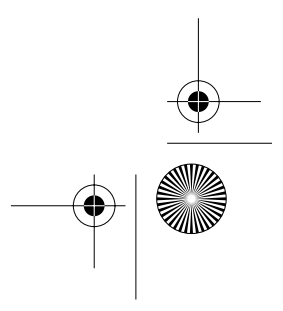


il 2004 (e soprattutto in quest'ultimo anno) ${ }^{44}$, la tendenza alla crescita del voto personale ai candidati sindaci subisce una battuta d'arresto: questo dato generale non può che riflettersi anche nei dati relativi alle performances dei sindaci, sia che si tratti di riconferme che di nuove elezioni. Inoltre, come abbiamo ricordato in precedenza, la tendenza all'allargamento delle coalizioni di sostegno alle candidature, costituisce di per sé un fattore di tendenziale abbassamento della quota di voti personali, rispetto ai voti di lista. Tuttavia, vi sono ulteriori specificità che rimandano, probabilmente, anche ad una diversa fase del governo degli enti locali: si potrebbe ipotizzare che il mestiere di sindaco, in questi anni, diventi più difficile e non assicuri più, immediatamente, una facile popolarità. Se ne scorgono forse alcuni segni nei dati a nostra disposizione. Cominciamo a guardare i casi di riconferma dei sindaci di centrosinistra e di centrodestra: per quanto riguarda il centrodestra, nei cinque casi considerati, l'IP scende da 0,17 a 0,16 , mentre l'IA rimane sostanzialmente stabile; per i sindaci di centrosinistra, invece, il dato medio mostra ancora una crescita dell'indice IP, da 0,08 a $0,10 \mathrm{e}$ una crescita notevole anche dell'indice IA (da 0,56 a 0,65 ).

Tuttavia, il ridotto numero dei casi ci consiglia uno sguardo più ravvicinato (TAB. 21). Emerge così un quadro molto più mosso: su tredici casi di sindaci di centrosinistra, ve ne sono ben sei in cui l'IP segna una flessione, tre casi in cui rimane stabile, e solo quattro casi in cui registra una crescita. In realtà, il dato medio viene influenzato dalla forte crescita di due comuni in particolare (Figline Valdarno e Viareggio): scorporandone i dati, anche la media degli IP resterebbe stabile $(0,07$ in entrambi i cicli). Anche per i cinque sindaci di centrodestra rieletti, il quadro è variegato: in due casi una crescita significativa dell'IP (Grosseto e Lucca), negli altri tre casi un regresso. Questi dati appaiono tanto più significativi se solo si considera che, nel precedente ciclo elettorale, nei 29 casi di riconferma dei sindaci eletti una prima volta nel 1993-1995, solo in cinque casi si era registrato una flessione dell'IP del sindaco incumbent ${ }^{45}$.

Se a questo quadro aggiungiamo i dati relativi ai casi di avvicendamento (ben 28, in gran parte dovuti alla conclusione del secondo mandato del sindaco in carica), ricaviamo una conferma della brusca frenata che ha subito, in quest'ultimo ciclo elettorale, la crescita del cosiddetto valore aggiunto dei candidati sindaco: un fenomeno che, come abbiamo visto, può avere come possibile spiegazione alcuni dati sistemici, legati ai meccanismi del sistema elettorale e ai processi di adattamento e apprendimento che essi hanno indotto nei comportamenti degli attori politici, ma che forse rimanda anche ad alcuni processi di carattere politico e istituzionale, su cui cercheremo di soffermarci più ampiamente nelle conclusioni di questo lavoro.

${ }^{44}$ Ricordiamo che, su 52 elezioni considerate, una sola si è svolta nel 2000, sei nel 2001, cinque nel 2002, tre nel 2003 e ben trentasette nel 2004.

45 Va rilevato anche che, nel 1997-1999, erano stati molto più frequenti (quasi la metà, 14 casi) le situazioni in cui diminuiva invece l'indice di attrazione dei sindaci rieletti, riflettendosi qui, evidentemente, la maggiore apertura competitiva delle elezioni svoltesi tra il 1997 e il 1999. 
TAв. 21 - Toscana. Sindaci ricandidati e rieletti. Indici di personalizzazione (IP) e di attrazione (IA). Comuni con oltre 15 mila abitanti.

Sindaci di centrosinistra

\begin{tabular}{|c|c|c|c|c|c|c|}
\hline \multirow[b]{2}{*}{ Comune } & \multirow{2}{*}{$\begin{array}{l}\mathrm{n}^{\circ} \text { liste collegate } \\
1997-1999\end{array}$} & \multirow{2}{*}{$\begin{array}{l}\mathrm{n}^{\circ} \text { liste collegate } \\
2000-2004\end{array}$} & \multicolumn{2}{|c|}{ 1997-1999 } & \multicolumn{2}{|c|}{$2000-2004$} \\
\hline & & & IP & IA & IP & IA \\
\hline Castelfiorentino & 4 & 5 & 0,08 & 0,70 & 0,05 & 0,75 \\
\hline Cecina & 6 & 6 & 0,12 & 0,69 & 0,11 & 0,76 \\
\hline Collesalvetti & 5 & 6 & 0,03 & 0,60 & 0,06 & 0,81 \\
\hline Figline Valdarno & 1 & 4 & 0,04 & 0,50 & 0,23 & 0,57 \\
\hline Firenze & 7 & 9 & 0,08 & 0,50 & 0,03 & 0,19 \\
\hline Monsummano Terme & 5 & 5 & 0,06 & 0,39 & 0,06 & 0,45 \\
\hline Montemurlo & 6 & 7 & 0,08 & 0,75 & 0,06 & 0,79 \\
\hline Pisa & 7 & 8 & 0,06 & 0,27 & 0,13 & 0,67 \\
\hline Poggibonsi & 2 & 3 & 0,06 & 0,71 & 0,06 & 0,80 \\
\hline Pontedera & 4 & 4 & 0,08 & 0,53 & 0,06 & 0,56 \\
\hline San Giovanni Valdarno & 4 & 3 & 0,08 & 0,55 & 0,07 & 0,73 \\
\hline San Miniato & 3 & 4 & 0,05 & 0,63 & 0,05 & 0,74 \\
\hline Viareggio & 5 & 6 & 0,14 & 0,48 & 0,36 & 0,57 \\
\hline
\end{tabular}

Sindaci di centrodestra

\begin{tabular}{|c|c|c|c|c|c|c|}
\hline \multirow[b]{2}{*}{ Comune } & \multirow{2}{*}{$\begin{array}{c}\mathrm{n}^{\circ} \text { liste collegate } \\
1997-1999\end{array}$} & \multirow{2}{*}{$\begin{array}{c}\mathrm{n}^{\circ} \text { liste collegate } \\
2000-2004\end{array}$} & \multicolumn{2}{|c|}{ 1997-1999 } & \multicolumn{2}{|c|}{$2000-2004$} \\
\hline & & & IP & IA & IP & IA \\
\hline Arezzo & 4 & 5 & 0,15 & 0,68 & 0,10 & 0,60 \\
\hline Grosseto & 5 & 7 & 0,16 & 0,76 & 0,24 & 0,67 \\
\hline Lucca & 4 & 6 & 0,17 & 0,36 & 0,22 & 0,65 \\
\hline Massarosa & 3 & 6 & 0,17 & 0,40 & 0,10 & 0,60 \\
\hline Montecatini Terme & 3 & 6 & 0,19 & 0,86 & 0,13 & 0,50 \\
\hline
\end{tabular}

\section{La struttura delle coalizioni e l'esito delle competizioni}

In conclusione, rivolgiamo la nostra attenzione all'esito della competizione ponendolo in relazione con la struttura e la composizione delle coalizioni. Abbiamo già visto sopra il quadro riassuntivo dei diversi cicli elettorali, con i diversi casi di conferme, alternanze e avvicendamenti.

Questo esito delle competizioni, tuttavia, è avvenuto in presenza di un quadro molto variegato di composizione delle coalizioni: come mostra la TAB. 22 , la struttura della competizioni tra i due primi candidati può essere riassunta attraverso una serie di combinazioni ${ }^{46}$. In particolare, abbiamo distinto $i$ casi in

\footnotetext{
${ }^{46}$ I primi due candidati, quasi sempre, sono espressione delle due maggiori coalizioni. Solo in due casi, nel primo ciclo elettorale, e in uno, nel secondo e nel terzo, vi è stata una sfida "tutta a sinistra", tra il candidato di centrosinistra e quello di Rifondazione
} 
cui le due maggiori coalizioni si sono presentate al completo, cioè senza defezioni di una o più delle proprie componenti, e $\mathrm{i}$ casi in cui una di esse, o entrambe, scontavano la presenza autonoma, con un proprio candidato sindaco, di altre liste riconducibili allo stesso schieramento. Per il centrosinistra, inoltre, abbiamo distinto, i casi in cui nella coalizione era presente anche Rifondazione comunista. Ne risultano dunque sei modelli-base di competizione bipolare, ai quali abbiamo dovuto aggiungere altre combinazioni che, soprattutto per il primo ciclo elettorale, hanno avuto una certa rilevanza.

Dai dati emerge con grande nettezza la progressiva strutturazione in senso bipolare dei sistemi politici locali della Toscana. Il primo ciclo elettorale, (specie con le elezioni svoltesi nel 1993-1994) mostra ancora una grande varietà di combinazioni coalizionali: in particolare, in molti casi, è ancora ben visibile quella struttura tripolare che caratterizzò anche le elezioni politiche del 1994, con una presenza autonoma della DC (nel 1993) e poi del PPI e del Patto per l'Italia. Inoltre, la fluidità che caratterizzava la struttura dei partiti, in quella fase, si riflette anche nella scelta, piuttosto diffusa, compiuta dal PDS di correre da solo o insieme ad altre singole formazioni politiche della sinistra, contrapponendosi o a coalizioni centriste o, spesso, a coalizioni locali in cui confluivano le varie componenti del centrodestra. Da ciò, anche, l'elevato numero di ballottaggi, notevolmente ridottosi poi con i successivi cicli elettorali.

Nelle elezioni degli anni 1997-1999 l'evoluzione bipolare prosegue e anche i processi di apprendimento sul nuovo sistema elettorale producono i propri effetti, consigliando agli attori politici la formazione di larghe coalizioni, (e sin dal primo turno, risultando poi complesse, e non sempre andando a buon fine, le trattative per gli eventuali, successivi apparentamenti). Si dimezzano conseguentemente i casi di ballottaggio (da 19 a 8). Nel $43 \%$ dei casi la sfida si configura secondo un formato "standard", con l'intero centrosinistra (senza Rifondazione) contrapposto all'intero centrodestra. Nel 21\% dei casi la coalizione di centrosinistra comprende anche Rifondazione. In altri 10 casi (17\%), la coalizione di centrosinistra si presenta incompleta, mancando (oltre a Rifondazione) qualche altra componente (quasi sempre i Verdi). Nel complesso appare più coesa la coalizione di centrodestra: in 35 comuni su 51 (ovvero, nel 68\% dei casi) si presenta senza defezioni di una o più componenti. Nel caso del centrosinistra, a far crescere i casi di presentazione completa della coalizione, contribuisce soprattutto la totale scomparsa dei casi, ancora frequenti nel 1993-1995, in cui ad opporsi al centrodestra, o a coalizioni centriste, erano le sole componenti di sinistra, o anche in alcuni casi, il solo PDS (ad esempio, a Siena). I dati del 1997 registrano un consolidarsi del profilo di centrosinistra della coalizione, con una presenza stabile e diffusa delle componenti di matrice cattolica: una novità, occorre ricordare, nel panorama del governo locale di una regione come la Toscana, in cui storicamente si è sempre potuto parlare di giunte rosse, o di governo locale della sinistra. 

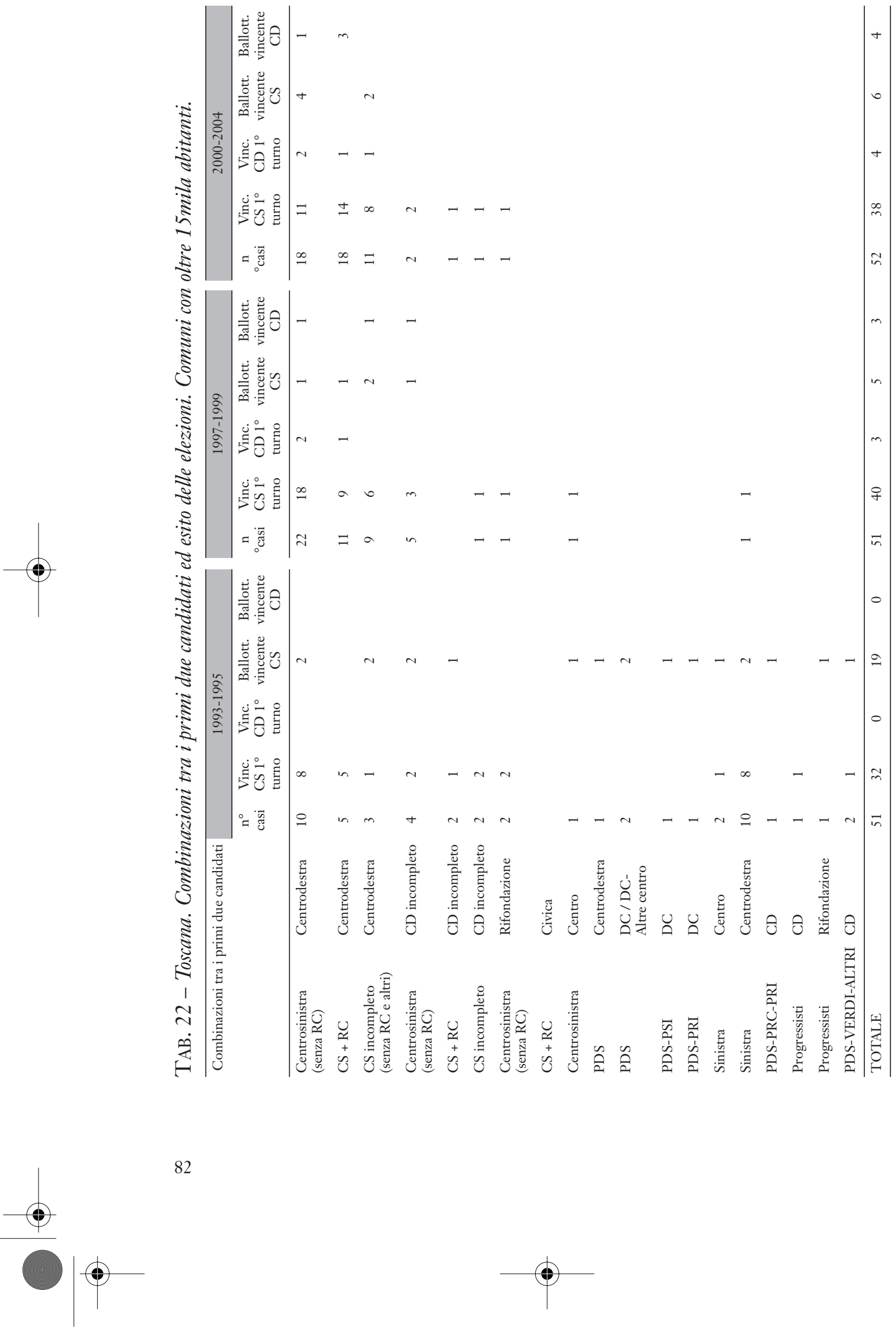
Con il terzo ciclo elettorale diminuiscono leggermente le coalizioni di centrosinistra senza Rifondazione e crescono invece quelle in cui questo partito è presente: nel complesso, 20 casi, a fronte di 19. Salgono a 11 i casi in cui il centrosinistra si presenta incompleto (nel senso sopra specificato): oltre ai Verdi, anche altri partiti (sulla base di dinamiche locali) rompono e presentano un proprio candidato ${ }^{47}$. Il centrodestra mantiene stabile e completa la composizione della propria coalizione, in due comuni su tre.

Emerge dunque, nel complesso, una progressiva stabilizzazione in senso bipolare della struttura competitiva; ma anche il perdurare (all'incirca in un terzo dei comuni interessati) di tensioni locali che portano una o più componenti ad una presenza autonoma e talvolta piuttosto conflittuale.

La TAB. 23 ci mostra il quadro completo della composizione delle coalizioni vincenti. Per quanto riguarda il centrosinistra, è evidente la tendenza ad un allargamento, ma anche ad una notevole frammentazione, della coalizione: se nel primo ciclo elettorale erano numerose le liste unitarie e, in ogni caso, in circa $4 / 5$ dei casi, la coalizione vedeva al massimo tre liste (con il PDS, ovviamente, a fare da pivot della coalizione, presente in tutti i casi), con il successivo ciclo elettorale la situazione quasi si rovescia, e in circa $2 / 3$ dei casi, sono almeno quattro le liste che compongono la coalizione vincente.La tendenza prosegue con il terzo ciclo elettorale, quando i casi di coalizione con almeno quattro liste giungono all' $80 \%$ e numerosissime sono anche le situazioni con sei o più liste di sostegno al sindaco eletto.

Come poi emerge dalla ТАВ. 24, in cui si distinguono i casi in cui i vari partiti presentano un proprio autonomo candidato, nel passaggio tra i primi due cicli elettorali, si riducono notevolmente le presenze isolate di candidati di area PPI (da 15 ad uno solo), si dimezzano i casi di candidati dei Verdi (da 13 a 6), e abbastanza diffusa risulta la presenza autonoma di candidati dello SDI (8 casi), mentre rimangono frequenti, ed anzi crescono, le situazioni in cui Rifondazione corre da sola (da 30 a 33), a conferma di una difficoltà nei rapporti tra i due partiti che hanno raccolto l'eredità del PCI.

Molto più variegata la situazione con il terzo ciclo elettorale, quando più frequenti diventano i casi di candidati sostenuti, oltre che da Rifondazione, dai Verdi o da altre liste locali di sinistra ${ }^{48}$.

Molto più compatta, generalmente, la coalizione di centrodestra: i casi in cui una componente si presenta in modo autonomo sono pochi e riguardano soprattutto l'UDC e il Nuovo PSI (la cui collocazione nella coalizione di centrodestra, peraltro, specie nelle varie situazioni locali, non può considerarsi stabile o organica). Da rilevare poi, come nei casi in cui il centrodestra risulta vincente, solo in due casi (uno nel 1995-1997 e l'altro nel ciclo successivo) la coalizione si pre-

\footnotetext{
47 I casi più rilevanti sono stati quelli della Margherita a Viareggio, del Pdci a Empoli (con Rifondazione comunista invece alleata ai DS), dei Verdi a Livorno.

${ }^{48}$ Il caso più noto è quello di Firenze, con la candidata Ornella De Zordo sostenuta, oltre che da Rifondazione comunista, da altre due liste civiche espressione di realtà locali di movimento.
} 
senti composta solo dai partiti nazionali: negli altri casi, è presente comunque almeno una lista civica (addirittura tre, nel caso di Grosseto), a conferma di come questo tipo di liste giochi un ruolo importante nell'allargamento delle coalizioni e nel sostegno ai candidati sindaci.

ТАВ. 23 - Toscana. Composizione delle coalizioni vincenti, per ciclo elettorale. Comuni con oltre 15 mila abitanti.

\begin{tabular}{|c|c|c|c|}
\hline Ciclo elettorale & $1993-1995$ & $1997-1999$ & $2000-2004$ \\
\hline n. Comuni & 51 & 51 & 52 \\
\hline \multicolumn{4}{|l|}{ Coalizione vincente } \\
\hline CENTROSINISTRA & 51 & 45 & 44 \\
\hline \multicolumn{4}{|l|}{ di cui } \\
\hline Liste unitarie senza $P R C$ & 8 & 2 & \\
\hline Liste unitarie $C S+P R C$ & 4 & 1 & 1 \\
\hline Liste unitarie $C S+P D C I$ & & 2 & 1 \\
\hline Liste unitarie $C S+P R C+P D C I$ & & 1 & \\
\hline Liste unitarie $C S+P D C I+I d V$ & & & 1 \\
\hline Liste unitarie $C S+P R C+$ altri & 1 & & \\
\hline Liste unitarie sinistra $+P P I$ (o unitarie di centro) & 3 & 1 & \\
\hline solo PDS & 3 & & \\
\hline$P D S+P R C$ & 4 & & \\
\hline$P D S+$ una sola altra lista & 5 & 2 & \\
\hline$P D S+$ altre due liste & 13 & 5 & 5 \\
\hline$P D S+$ altre tre liste & 4 & 8 & 6 \\
\hline$P D S+$ altre quattro liste & 5 & 11 & 11 \\
\hline$P D S+$ altre cinque o più liste & 1 & 12 & 19 \\
\hline TOTALE & 51 & 45 & 44 \\
\hline CENTRODESTRA & - & 6 & 8 \\
\hline \multicolumn{4}{|l|}{ di cui } \\
\hline$F I-A N-C C D / C D U(U D C)+$ tre liste locali & & 1 & \\
\hline FI-AN-CCD $(U D C)+$ una lista locale & & 3 & 1 \\
\hline Forza Italia - CCD - locale & & 1 & \\
\hline$F I-A N-C C D$ & - & 1 & \\
\hline$F I-A N-U D C$ & & & 1 \\
\hline$F I-A N-C C D / C D U-L E G A+L O C A L E$ & & & 1 \\
\hline FI-AN-CCD/CDU-LEGA-N.PSI & & & 1 \\
\hline FI-AN-CCD/CDU-LEGA-N.PSI+PENS + LOC & & & 1 \\
\hline FI-AN-CCD/CDU + LEGA + due liste locali & & & 3 \\
\hline
\end{tabular}


TAB. 24 - Toscana. Presenze autonome di candidati. Comuni con oltre 15 mila abitanti.

\begin{tabular}{|c|c|c|c|}
\hline \multirow[b]{2}{*}{ Ciclo elettorale } & \multicolumn{3}{|c|}{$\begin{array}{l}\text { Numero di presenze con proprio candidato, } \\
\text { distinto dalla coalizione }\end{array}$} \\
\hline & $1993-1995$ & $1997-1999$ & $2000-2003$ \\
\hline$\overline{P R C}+$ ALTRA LOCALE & & & 4 \\
\hline PRC + VERDI & & & 3 \\
\hline $\mathrm{PRC}+\mathrm{SDI}+\mathrm{VERDI}+\mathrm{IDV}$ + LOC & & & 1 \\
\hline PRC & 30 & 33 & 18 \\
\hline VERDI & 13 & 6 & 3 \\
\hline PRC + PDCI + VERDI & & & 1 \\
\hline MARGHERITA & & & 1 \\
\hline PPI & 15 & 1 & \\
\hline DEMOCRATICI & 1 & 2 & \\
\hline $\mathrm{LAB}$ & 1 & & \\
\hline SDI & & 8 & \\
\hline PATTO & 4 & 1 & \\
\hline PDCI & & 1 & \\
\hline PDCI + ALTRA LOCALE & & & 1 \\
\hline IDV & & & 1 \\
\hline UDEUR & & 1 & 2 \\
\hline FI-LEGA-NUOVO PSI & & & 1 \\
\hline FI-UDC & & & 1 \\
\hline AN & & 1 & 1 \\
\hline AN-UDC & & & 1 \\
\hline AN-FI & & & 1 \\
\hline \multicolumn{4}{|l|}{ FI } \\
\hline UDC & & & 3 \\
\hline UDC-NUOVO PSI & & & 1 \\
\hline NUOVO PSI & & & 6 \\
\hline \multicolumn{4}{|l|}{ CCD } \\
\hline LEGA & & 1 & \\
\hline $\mathrm{CDU}$ & & 1 & \\
\hline
\end{tabular}




\section{Un bilancio positivo e un difficile equilibrio}

Obiettivo di questo lavoro era quello di analizzare i meccanismi di funzionamento della nuova legge elettorale nel contesto specifico della Toscana (o meglio, dei 51 Comuni toscani con oltre 15 mila abitanti) e di proporre una prima valutazione degli effetti che il nuovo contesto istituzionale ha prodotto e sta producendo sui sistemi politici locali di questa regione, nell'arco dei tre cicli elettorali considerati. In conclusione, possiamo dunque chiederci: la nuova legge elettorale ha raggiunto gli obiettivi che si proponeva? $\mathrm{O}$, in modo più circoscritto, data l'evidente diversità territoriale e regionale dell'impatto della nuova legge nel contesto italiano, essa come ha funzionato in Toscana?

Generalmente, una possibile risposta a questi interrogativi, che trova ampi consensi e che risulta anche confermata dall'analisi del caso toscano, suona all'incirca così: la nuova legge ha certamente risposto positivamente alle esigenze di stabilità e di responsabilizzazione dei governi locali, ha permesso di rompere decisamente con il passato, quando i sindaci erano permanentemente sotto scacco, le crisi delle giunte frequenti e comunque sempre incombenti, l'orizzonte temporale delle politiche pubbliche locali ristretto e viziato da una logica particolaristica di ricerca e riproduzione del consenso 49 .

Un tale obiettivo di stabilizzazione del potere locale è stato perseguito anche attraverso un più elevato tasso di personalizzazione della politica, alimentando e assecondando le spinte che, peraltro, muovevano in tale direzione all'interno dello scenario politico nazionale, sia con una forte personalizzazione delle leadership delle due coalizioni e l'introduzione dei collegi uninominali, in occasione delle elezioni politiche, sia con analoghe riforme che investivano altri livelli istituzionali, come le Regioni: l'elezione diretta del sindaco, e la nuova centralità che la figura dei sindaci ha così acquistato, rappresentano senza dubbio una novità istituzionale di grande rilievo, ben difficilmente reversibile, che probabilmente non ha ancora esaurito tutti i suoi possibili effetti sull'intero assetto del nostro sistema politico.

Certo, oggi siamo ancora di fronte ad un difficile e incerto equilibrio: alla prova dei fatti, le nuove regole si sono trovate a cercare una mediazione tra le spinte ad una maggiore personalizzazione della politica e le esigenze volte a preservare un ruolo al confronto politico e programmatico tra i partiti e le coalizioni. Un equilibrio precario, e sostanzialmente irrisolto, in cui peraltro si riflette la stessa logica che ha ispirato sin dall'inizio il processo riformatore e che è ben visibile nei suoi esiti: le nuove regole hanno accolto, e insieme hanno alimentato e contribuito a riprodurre, quel peculiare bipolarismo frammentato che sembra costituire tuttora la cifra dominante del nostro sistema politico, nella lunga e inde-

${ }^{49}$ Nel periodo 1971-1989 la durata media delle giunte nei comuni capoluogo era stata inferiore ai due anni: si veda F. CAZZOLA, Periferici e integrati. Chi, dove e quando nelle amministrazioni locali, Bologna, Il Mulino, 1991. 
finita transizione italiana. In particolare, la scelta di puntare sull'elezione diretta dei sindaci, e di privilegiare come arena decisiva dello scontro politico ed elettorale la scelta degli esecutivi, va vista e interpretata insieme alla crisi e alla destrutturazione che il sistema dei partiti ha vissuto negli anni Novanta e che non sembrano ancora giunte ad un qualche esito stabile o ben definibile: è in questo snodo che va colto l'impatto più profondo delle nuove regole. Ma proprio la diversa natura e le varie caratteristiche del sistema dei partiti e dei sistemi politici locali, nelle diverse regioni del paese, ha prodotto effetti differenziati, che giustificano una dimensione regionale dell'analisi.

Per la Toscana, il punto cruciale da sottoporre a verifica riguarda in primo luogo l'impatto di questo nuovo contesto istituzionale su un sistema politico che, a lungo, com'è noto, è stato interpretato alla luce di una peculiare categoria, quella di sub-cultura politica territoriale. Senza poter entrare qui nel merito delle profonde trasformazioni che hanno investito il complesso degli elementi che vengono richiamati da questa nozione, appare però necessario richiamare alcuni aspetti che incidono più direttamente sul nostro argomento ${ }^{50}$.

Un tassello fondamentale del modello originario delle "regioni rosse" era costituito non solo dalla continuità dei comportamenti elettorali (in larga misura riconducibili alla tipologia del voto di appartenenza), ma dall'esistenza di una densa rete associativa e istituzionale fondata su una comune matrice politico-culturale, con un forte ruolo di coordinamento e di direzione svolto dalla forza politica egemone. $\grave{E}$ qui che possiamo per intero misurare la portata delle trasformazioni intervenute e lo stesso impatto del nuovo contesto istituzionale prodotto dalla nuova legge elettorale: «un sistema politico caratterizzato dal predominio di un partito, da una robusta organizzazione della società civile e da un'elevata capacità di mediazione dei diversi interessi» ${ }^{51}$, ha lasciato il posto ad un sistema politico in cui, certamente, continua a dominare una cultura politica di fondo orientata a sinistra, in cui è certamente tuttora robusta l'organizzazione della società civile (per quanto diversamente strutturata e ricca di autonomie) ed in cui permane elevata la capacità di mediazione e di rappresentanza degli interessi (per quanto diversamente praticata, rispetto al passato), ma in cui è venuto sicuramente meno un pilastro del precedente modello, ossia il ruolo del partito dominante come perno dell'intero sistema politico locale.

${ }^{50}$ Il lavoro che per la prima volta affrontò compiutamente il tema delle "subculture politiche territoriali" è stato quello di C.TRIGILIA, Grandi partiti e piccole imprese, Bologna, Il Mulino, 1986. Un recente volume di F. RAMELLA, Cuore rosso? Viaggio politico nell'Italia di mezzo, Roma, Donzelli, 2005, oltre a riproporre un'efficace sintesi dei tratti costitutivi del modello politico-istituzionale ed economico-sociale delle "regioni rosse", analizza dettagliatamente i processi evolutivi di questo modello, i fattori di crisi da cui è stato investito come pure i punti di forza che continuano a caratterizzarlo. Sulla peculiare posizione della Toscana, nella crisi degli anni Novanta, e sulle trasformazioni della subcultura rossa, ci permettiamo di rinviare anche a A.FLORIDIA, «Le "risorse istituzionali" dello sviluppo locale: la Toscana e i sentieri divergenti della Terza Italia», in Sviluppo locale, 12, 1999, pp. 79-104.

${ }^{51}$ F. RAMELla, Cuore rosso?, cit., p. 26. 
In effetti, non siamo di fronte al precipitare improvviso di un cambiamento: già gli studi più attenti condotti negli anni Ottanta avevano sottolineato come il riprodursi di un largo consenso alla sinistra fosse sempre più condizionato e legato alla capacità di rappresentanza sociale degli interessi e alle funzioni di mediazione istituzionale tra le forze sociali svolte dal governo locale; e come sempre meno esso potesse contare sullo spontaneo perpetuarsi degli antichi collanti politico-ideologici, che tanta parte avevano avuto nel favorire l'originario insediamento subculturale e la costruzione di un'identità collettiva, almeno fino a tutti gli anni Cinquanta e Sessanta, e forse ancora oltre. Insomma, alla base del perdurante voto a sinistra, già allora, si poteva cogliere un progressivo slittamento delle forme del consenso, verso forme di delega più condizionate e strumentali, sempre meno immediatamente riconducibili alle matrici comunitarie originarie e ad una logica di appartenenza. Oggi questo processo, una sorta di lenta mutazione, sembra giunto ad una sua compiuta maturazione, esprimendosi pienamente anche sul piano elettorale; e non tanto perché i rapporti di forza appaiano stravolti, rispetto al passato, ma per una ragione più di fondo: sono molti i sintomi che ci fanno dire, oggi, che il mercato elettorale toscano si presenta come un mercato molto più aperto che in passato, dove contano e conteranno sempre più, di volta in volta, le specifiche caratteristiche dell'offerta politica che gli schieramenti sapranno mettere in gioco e dove è oramai possibile interpretare i comportamenti degli elettori sotto il segno dello «scongelamento» ${ }^{52}$.

Di tutto ciò abbiamo colto, riteniamo, un forte riflesso anche nelle pagine di questo nostro lavoro: basti pensare, prima di tutto, alla stessa partecipazione elettorale, la cui evoluzione, nelle sue forme e nei suoi livelli, porta i segni di una profonda trasformazione politica e culturale. Una nuova dimensione che, specie nelle elezioni amministrative, è spesso decisiva nel segnare l'esito del voto.

In questo mutato scenario, il nuovo sistema elettorale e la nuova forma del governo locale hanno inciso profondamente: in un certo senso, hanno contribuito ad accelerare e ad orientare processi di mutamento che avevano radici e ragioni proprie, ma che forse, in assenza di un nuovo contesto istituzionale, avrebbero faticato ad affermarsi. In particolare, l'innovazione dell'elezione diretta del sindaco, unita ai processi di indebolimento e destrutturazione del sistema dei partiti (che anche in Toscana si sono manifestati, sia pure in misura diversa rispetto ad altre regioni del paese), ha prodotto un radicale spostamento del baricentro della rappresentanza politica locale: sono i sindaci ad essere oggi, molto più che in passato, la figura centrale di una comunità locale, collocati in una posizione nevralgica che consente loro di intessere una fitta trama di relazioni con una società

${ }^{52}$ F. Ramella, nel suo lavoro, propone una periodizzazione della storia elettorale delle "regioni rosse": gli anni del radicamento (1946-1958), gli anni della crescita (1958-1976), gli anni della stagnazione e del declino (1976-1992), a cui seguono gli anni del ricompattamento (1992-1996) e appunto quelli, che viviamo tuttora, dello scongelamento (1996-2001), precisando come si tratti «di uno scongelamento dei comportamenti di voto, non di un crollo delle tradizioni politiche precedenti, né dei partiti che le hanno interpretate» (p. 110). 
sempre più differenziata, che nessun altro soggetto sociale e politico, anche per la sua intrinseca parzialità, è ormai in grado di costruire e alimentare.

Questa nuova situazione presenta molte potenzialità, ma anche alcuni rischi. A distanza di dodici anni dalla riforma del 1993, cominciano ad essere possibili i primi bilanci dell'esperienza dei "nuovi sindaci", al di là anche dell'impatto specifico delle nuove regole elettorali. In generale, come abbiamo detto all'inizio di queste conclusioni, si può dire che sia stato raggiunto uno dei primi obiettivi che la riforma si era posto, ossia quello di dare maggiore stabilità al governo degli enti locali; ma nuovi problemi sono emersi, e sempre più emergono con nettezza.

La personalizzazione della politica locale colloca i sindaci ai vertici della dinamica decisionale; ma non sempre tale posizione di centralità si coniuga con sufficienti capacità e sensibilità politiche, comunicative e relazionali. Disporre di maggiori poteri decisionali non vuol dire capacità effettiva di esercitarli, o capacità di coinvolgere attivamente attori e soggetti sociali nella costruzione di una strategia di sviluppo locale. Spesso si è creata una sorta di strettoia istituzionale, da cui non sempre i sindaci sono stati in grado di uscire positivamente: e, in questo senso, come abbiamo notato più volte nel corso del nostro lavoro, ha pesato e pesa certo anche il nodo irrisolto del ruolo dei consigli comunali, luogo dove si esprime quella perdurante frammentazione della rappresentanza politica su cui la nuova legge elettorale non solo non ha inciso, ma che anzi ha contribuito ad alimentare.

Nel complesso, emerge con sempre maggiore nettezza quella che gli studiosi autori di una recente ricerca hanno definito come l'«illusione decisionistica» che ha accompagnato la riforma del 1993, l'illusione cioè che bastasse sottrarre i "nuovi sindaci" al peso soffocante dei partiti e delle correnti, o della burocrazia, perché d'incanto la politica locale potesse produrre buone politiche locali ${ }^{53}$. Non è così, o almeno stando ad un'analisi comparata di diverse situazioni locali, non sempre è stato ed è così: la personalizzazione della politica non basta, i nuovi sindaci hanno dovuto pagare spesso il prezzo della rarefazione dei canali di mediazione e di rappresentanza sociale degli interessi, e hanno misurato anche il vuoto lasciato dal venir meno di partiti che svolgessero bene il loro compito di filtro e di selezione della domanda politica. Il bisogno di una politica diffusa e di una politica radicata nel territorio, torna così a emergere prepotentemente. E, con esso, torna a riproporsi il tema del ruolo dei partiti, essendo ben presto svanita l'illusione che, ad un tale vuoto, si potesse opporre il protagoni-

${ }^{53}$ Ci riferiamo all'ampia ricerca curata da R. CATANZaro, F. Piselli, F. RAMELla, e C. TRIGILIA, che ha analizzato da vicino la vicenda politica e amministrativa di alcuni comuni, cercando di mettere a fuoco gli effetti del nuovo quadro istituzionale e politico degli enti locali negli anni Novanta, Comuni nuovi. Il cambiamento nei governi locali, Bologna, Il Mulino 2002. Proprio per questo, e per evidenti motivi, questo salto qualitativo positivo risaltava maggiormente in alcuni comuni meridionali, rispetto a quanto poteva registrarsi in altre aree del paese, tra cui la Toscana, che comunque avevano alle spalle una certa tradizione di buona amministrazione. 
smo politico dei movimenti della società civile: il caso della Toscana, da questo punto di vista, appare significativo, proprio in quanto la ricchezza della trama associativa ben viva in questa regione trova oggi alimento in un rapporto con la politica e le istituzioni locali fondato sulla reciproca autonomia, ed anche sul conflitto, ma certo non su un ruolo di supplenza.

D'altro canto, proprio i casi di successo dei nuovi sindaci mostrerebbero forse, ad un'analisi più ravvicinata del rapporto tra politica e politiche locali, come sia stata probabilmente la capacità di costruire una rete di relazioni più ampia con la società locale e gli interessi che vi si esprimono, e la capacità di instaurare un rapporto positivo con le stesse forze politiche e con la burocrazia, ad aver rappresentato la carta vincente di numerose esperienze di governo locale. $\grave{E}$ un tema complesso, che qui possiamo solo evocare, attorno a cui sarebbe necessario svolgere indagini specifiche, sul campo delle attività quotidiane di un'amministrazione comunale.

Insomma, siamo di fronte ad uno scenario che, forse, reca in sé i segni di una nuova fase di mutamento, contraddittoria ed aperta ai più diversi sviluppi. $\mathrm{E}$ un sintomo di tutto ciò può essere forse colto anche in alcuni elementi della nostra analisi. Come abbiamo visto, con il terzo ciclo elettorale, e con le elezioni del 2004 in particolare, anche la crescita del voto personalizzato ai candidati sindaco sembra essersi arrestata. La scelta di privilegiare solo la figura dei candidati riguarda una quota significativa, ma pur sempre minoritaria, degli elettori. Si tratta, beninteso, di una quota che può rivelarsi decisiva, specie quando la competizione tra gli schieramenti è serrata; ma che non intacca il ruolo chiave giocato dal consenso ai partiti e alle coalizioni. Altri soggetti, vecchi e nuovi, sono entrati in gioco: per un verso, (almeno in Toscana, sicuramente), i partiti sembrano recuperare un ruolo maggiore nella rappresentanza degli interessi locali e nella formazione e trasmissione del consenso. Sembra avviata a chiudersi, forse, una fase caratterizzata da quella "cultura dell'antipolitica" che vedeva i partiti come strumenti oramai obsoleti e presupponeva una società civile costretta ad affidarsi soltanto alle virtù personali dei candidati, chiamati ad un arduo compito di supplenza, nella costruzione della rappresentanza. Torna a contare il territorio, la presenza organizzata, la capacità di insediamento sociale: un buon candidato sindaco può essere il catalizzatore di tutti questi fattori, può esaltarne la ricchezza, ma non può supplire, da solo, ad una loro debolezza.

Anche i dati più recenti (per quanto parziali), che sembrano mostrare un indebolimento delle riserve di consenso personale che gli stessi sindaci incumbents, se pure rieletti, riescono a conservare, possono forse essere letti in questo senso: ovvero, il mestiere di sindaco si fa più difficile, e amministrare non è un compito agevole che possa garantire facili e immediati consensi o ritorni di immagine. Sono stati anni difficili per i governi locali, per tante ragioni che qui non serve richiamare: e anche quando (e in Toscana, gli esempi non mancano), $\mathrm{i}$ comuni affrontano complesse azioni di trasformazione strutturale delle città, la resa e la visibilità di questi mutamenti non è, e non può essere immediata; e le 
incomprensioni, le difficoltà di dialogo con i cittadini, incombono ${ }^{54}$. E del resto, proprio in ciò sta il fondamentale merito della nuova legge elettorale approvata nel 1993: aver garantito stabilità ai governi locali, essenziale pre-requisito perché sindaci e giunte possano, quando ne siano capaci, misurarsi anche su politiche proiettate sul medio-lungo periodo. $\grave{E}$ un elemento che va ricordato, specie a quanti coltivano incomprensibili nostalgie assembleari, dimenticando l'epoca in cui sindaci e giunte erano costantemente sotto scacco, preda di consigli comunali in cui furoreggiava una contrattazione permanente tra partiti, correnti e sotto-correnti.

Accanto a queste spiegazioni di ordine politico e istituzionale, a frenare il voto personalizzato ai sindaci concorrono anche gli specifici meccanismi previsti dal sistema elettorale, come abbiamo più volte ricordato nelle pagine precedenti. In un recente passato, proprio la crisi e la debolezza dei partiti ha esaltato, in molti casi, la forza e l'autorevolezza dei candidati. Oggi, come si diceva sopra, e specie in una realtà come quella toscana, esistono alcuni segni tangibili di un possibile, ritrovato ruolo dei partiti, con una più elevata capacità di radicamento e mobilitazione. Tutto ciò non può che incidere sul ruolo dei candidati e dei sindaci: se in passato poteva accadere di avere buoni e anche ottimi candidati, il cui rendimento positivo veniva però accentuato dalla debolezza dei partiti, oggi sembra profilarsi una tendenza opposta: candidati che, in genere, risultano buoni, o anche ottimi, ma il cui valore aggiunto non risalta più come in passato, avendo alle spalle, per un verso, coalizioni ampie e variegate e un numero crescente di liste; e, per altro verso, partiti sicuramente un po' più strutturati, con un insediamento organizzativo che, per quanto non paragonabile a quello del passato, garantisce comunque una maggiore capacità di selezione della domanda sociale e di raccolta del consenso. Insomma, il sindaco continua ad essere il baricentro della rappresentanza politica locale, e ben difficilmente si potrà tornare indietro, da questo punto di vista; ma, forse, non potrà più essere, come pure per una fase è apparso plausibile, il dominus incontrastato della politica locale, l'unico (e il più visibile) avamposto a cui si rivolge una domanda sociale esigente, mutevole e spesso anche contraddittoria. Non potrà più esserlo: e probabilmente è anche più giusto e "sano", per la nostra democrazia locale, che sia così.

\footnotetext{
${ }^{54}$ Le ricerche contenute nel sopra citato volume sui «comuni nuovi» hanno mostrato come, in molti casi, la percezione di un miglioramento nella qualità del governo locale, rispetto alla fase preriforma, nel giudizio degli elettori e degli opinion leaders locali, investiva soprattutto una serie di politiche di più diretto impatto sulla vita quotidiana dei cittadini, e molto meno quelle sfere (ad esempio, le grandi scelte urbanistiche), dove tale impatto risulta difficilmente "visibile" nel breve periodo.
} 